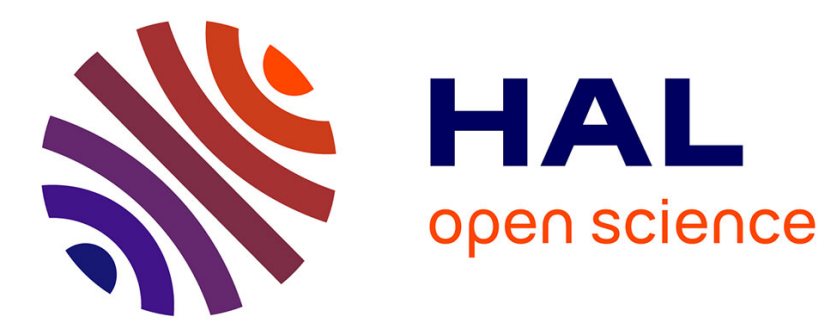

\title{
Pluto's Sputnik Planitia: Composition of geological units from infrared spectroscopy
}

F. Scipioni, O. White, J.C. C Cook, T. Bertrand, D.P. P Cruikshank, W.M.

M Grundy, C. Beddingfield-Cartwright, R.P. P Binzel, C.M. M Dalle Ore, D. Jennings, et al.

\section{To cite this version:}

F. Scipioni, O. White, J.C. C Cook, T. Bertrand, D.P. P Cruikshank, et al.. Pluto's Sputnik Planitia: Composition of geological units from infrared spectroscopy. Icarus, 2021, 359, pp.114303. 10.1016/j.icarus.2021.114303 . hal-03312888

\section{HAL Id: hal-03312888 https://hal.science/hal-03312888}

Submitted on 3 Aug 2021

HAL is a multi-disciplinary open access archive for the deposit and dissemination of scientific research documents, whether they are published or not. The documents may come from teaching and research institutions in France or abroad, or from public or private research centers.
L'archive ouverte pluridisciplinaire HAL, est destinée au dépôt et à la diffusion de documents scientifiques de niveau recherche, publiés ou non, émanant des établissements d'enseignement et de recherche français ou étrangers, des laboratoires publics ou privés. 
${ }^{1}$ SETI Institute, Mountain View, CA, 94040, USA

${ }^{2}$ NASA Ames Research Center; Moffett Field, CA, 94035, USA

${ }^{3}$ Pinhead Institute, Telluride, CO, USA

${ }^{4}$ Lowell Observatory, Flagstaff, AZ, 8600, USA

${ }^{5}$ Massachusetts Institute of Technology, Cambridge, MA 02139, USA

F. Scipioni ${ }^{1}$, O. White ${ }^{1,2}$, J.C. Cook ${ }^{3}$, T. Bertrand ${ }^{2}$, D.P. Cruikshank², W.M. Grundy ${ }^{4}$, C. Beddingfield-Cartwright $^{1}$, R. P. Binzel ${ }^{5}$, C.M. Dalle Ore ${ }^{1,2}$, D. Jennings ${ }^{6}$, J.M. Moore ${ }^{2}$, C.B. Olkin $^{7}$, S. Protopapa ${ }^{7}$, D. C. Reuter ${ }^{6}$, B. Schmitt ${ }^{8}$, K.N. Singer ${ }^{7}$, J.R. Spencer ${ }^{7}$, S.A. Stern ${ }^{7}$, H.A. Weaver $^{9}$, A.J. Verbiscer ${ }^{10}$, L.A. Young ${ }^{7}$.

${ }^{7}$ Southwest Research Institute, Boulder, CO, 80302, USA

${ }^{8}$ Université Grenoble Alpes, CNRS, Grenoble, France

${ }^{9}$ Johns Hopkins University Applied Physics Laboratory, Laurel, MD, 20723, USA

${ }^{10}$ University of Virginia, Charlottesville, VA, 22904, USA 


\section{Abstract}

32 We have compared spectroscopic data of Sputnik Planitia on Pluto, as acquired by New Horizons'

33 Linear Etalon Imaging Spectral Array (LEISA) instrument, to the geomorphology as mapped by

34 White et al. (2017) using visible and panchromatic imaging acquired by the LOng-Range

35 Reconnaissance Imager (LORRI) and the Multi-spectral Visible Imaging Camera (MVIC). We

36 have focused on 13 of the geologic units identified by White et al. (2017), which include the plains

37 and mountain units contained within the Sputnik basin. We divided the map of Sputnik Planitia into

3815 provinces, each containing one or more geologic units, and we use LEISA to calculate the average

39 spectra of the units inside the 15 provinces. Hapke-based modeling was then applied to the average

40 spectra of the units to infer their surface composition, and to determine if the composition resulting

41 from the modeling of LEISA spectra reflects the geomorphologic analyses of LORRI data, and if

42 areas classified as being the same geologically, but which are geographically separated, share a

43 similar composition. We investigated the spatial distribution of the most abundant ices on Pluto's

44 surface $-\mathrm{CH}_{4}, \mathrm{~N}_{2}, \mathrm{CO}, \mathrm{H}_{2} \mathrm{O}$, and a non-ice component presumed to be a macromolecular carbon-rich

45 material, termed a tholin, that imparts a positive spectral slope in the visible spectral region and a

46 negative spectral slope longward of $\sim 1.1 \mu \mathrm{m}$. Because the exact nature of the non-ice component is

47 still debated and because the negative spectral slope of the available tholins in the near infrared does

48 not perfectly match the Pluto data, for spectral modeling purposes we reference it generically as the

49 negative spectral slope endmember (NSS endmember). We created maps of variations in the

50 integrated band depth (from LEISA data) and areal mass fraction (from the modeling) of the

51 components. The analysis of correlations between the occurrences of the endmembers in the geologic

52 units led to the observation of an anomalous suppression of the strong $\mathrm{CH}_{4}$ absorption bands in units

53 with compositions that are dominated by $\mathrm{H}_{2} \mathrm{O}$ ice and the NSS endmember. Exploring the mutual

54 variation of the $\mathrm{CH}_{4}$ and $\mathrm{N}_{2}$ integrated band depths with the abundance of crystalline $\mathrm{H}_{2} \mathrm{O}$ and NSS 
55 endmember revealed that the NSS endmember is primarily responsible for the suppression of

$56 \mathrm{CH}_{4}$ absorptions in mountainous units located along the western edge of Sputnik Planitia. Our

57 spectroscopic analyses have provided additional insight into the geological processes that have

58 shaped Sputnik Planitia. A general increase in volatile abundance from the north to the south of

59 Sputnik Planitia is observed. Such an increase first observed and interpreted by Protopapa et al. 2017

60 and later confirmed by climate modeling (Bertrand et al., 2018) is expressed geomorphologically in

61 the form of preferential deposition of $\mathrm{N}_{2}$ ice in the upland and mountainous regions bordering the

62 plains of southern Sputnik Planitia. Relatively high amounts of pure $\mathrm{CH}_{4}$ are seen at the southern

63 Tenzing Montes, which are a natural site for $\mathrm{CH}_{4}$ deposition owing to their great elevation and the

64 lower insolation they are presently receiving. The NSS endmember correlates the existence of tholins

65 within certain units, mostly those coating the low-latitude mountain ranges that are co-latitudinal

66 with the tholin-covered Cthulhu Macula. The spectral analysis has also revealed compositional

67 differences between the handful of occurrences of northern non-cellular plains and the surrounding

68 cellular plains, all of which are located within the portion of Sputnik Planitia that is presently

69 experiencing net sublimation of volatiles, and which do not therefore exhibit a surface layer of bright,

70 freshly-deposited $\mathrm{N}_{2}$ ice. The compositional differences between the cellular and non-cellular plains

71 here hint at the effectiveness of convection in entraining and trapping tholins within the body of the

72 cellular plains, while preventing the spread of such tholins to abutting non-cellular plains.

73 1. Introduction

74 In 2015 the New Horizons spacecraft encountered dwarf planet Pluto, unveiling a complex

75 and diverse icy world (Stern et al., 2015). Prior to New Horizons' flyby, Pluto had been

76 extensively studied from ground-based and space telescopes. Observations in the visible (VIS;

77 e.g., Lorenzi et al., 2016) and infrared range (IR; e.g. Cruikshank et al., 1976; Grundy et al.,

78 2013; 2014; Merlin, 2015, Olkin et al., 2007; Protopapa et al., 2008), determined that Pluto’s

* Some feature names mentioned in this paper are now formalized while others are informal 
uppermost surface layer is mostly composed of frozen $\mathrm{N}_{2}, \mathrm{CH}_{4}, \mathrm{CO}, \mathrm{C}_{2} \mathrm{H}_{6}$, along with additional

80 non-ice, organic components (Cruikshank et al., 2015). This surficial coating overlies bedrock

81 made of $\mathrm{H}_{2} \mathrm{O}$ ice.

82 The surface of Pluto exhibits distinct terrain types, the most conspicuous being Sputnik

83 Planitia*, a $1000 \mathrm{~km}$ by $1300 \mathrm{~km}$ wide impact basin that is interpreted to have formed $\gtrsim 4$ Gya

84 (Moore et al., 2016; McKinnon et al., 2016; Johnson et al., 2016; Keane et al., 2016; Nimmo et

85 al., 2016; Schenk et al., 2018). The deep basin represents a powerful cold trap and a natural

86 setting for the accumulation of volatile ices spread across Pluto's surface, principally $\mathrm{N}_{2}$ and $\mathrm{CO}$

87 ice (Bertrand \& Forget, 2016; Hamilton et al., 2016; Earle et al., 2017). Modeling of volatile

88 behavior in response to topography has shown that infilling of the basin with the majority of

89 surface $\mathrm{N}_{2}-\mathrm{CO}$ ice would be complete by tens of millions of years after its formation (Bertrand et

90 al., 2018). Most of the surface of Sputnik Planitia today is depressed by $2.5-3.5 \mathrm{~km}$ relative to the

91 basin rim, and by $2 \mathrm{~km}$ relative to Pluto's mean radius (Schenk et al., 2018). No impact craters

92 are seen within Sputnik Planitia in contiguous $315 \mathrm{~m} /$ pixel mapping coverage (Robbins et al.,

93 2017; White et al., 2017; Singer et al., 2019), indicating very recent resurfacing of its plains. The

94 central and northern portions of Sputnik Planitia are marked by a reticulate pattern of troughs a

95 few km wide that divide the plains into ovoid cells (Stern et al., 2015; Moore et al., 2016). The

96 cells likely originated due to solid-state convection of the $\mathrm{N}_{2}-\mathrm{CO}$ ice, with a surface renewal

97 timescale of <1 Myr (McKinnon et al., 2016; Trowbridge et al., 2016; Buhler \& Ingersoll, 2018).

98 Cells are absent within the southern and eastern parts of Sputnik Planitia, which instead display

99 featureless plains or dense concentrations of sublimation pits reaching a few $\mathrm{km}$ across (Stern et

100 al., 2015; Moore et al., 2016; White et al., 2017). A discontinuous chain of mountain ranges,

101 consisting of discrete angular blocks with apparently random orientations, extends for hundreds 
102 of kilometers along the western margin of Sputnik Planitia (Moore et al., 2016; Skjetne et al.,

103 2020). These are interpreted to be fragments of Pluto's tectonized and brecciated $\mathrm{H}_{2} \mathrm{O}$ ice crust

104 that were intruded by glacial $\mathrm{N}_{2}-\mathrm{CO}$ ice covering the uplands and flowing into the Sputnik basin

105 in the aftermath of the impact, and subsequently dislodged, transported and rotated by the glacial

106 ice to form the ranges (Moore et al., 2016; Stern et al., 2015; Howard et al., 2017; O'Hara \&

107 Dombard, 2020).

108 We report here a compelling analysis of the Sputnik Planitia region, performed by comparing

109 the data acquired by the LOng-Range Reconnaissance Imager (LORRI) (Cheng et al., 2008) with

110 the spectroscopic observations returned by the Linear Etalon Imaging Spectral Array (LEISA)

111 (Reuter et al., 2009). Our investigation has the goal to understand if the terrain units identified by

112 White et al. (2017) on the basis of geologic analysis, display distinct spectral characteristics in

113 addition to their geomorphology and albedo.

114

$115 \quad$ 2. Geologic and spectral mapping

116 A geological map of Sputnik Planitia was produced by White et al. (2017), using a mosaic of

11712 LORRI images as the base map. The base map covers the plains themselves and the

118 surrounding uplands. The mapping defined 15 geologic units, based primarily on the surface

119 texture and albedo that they present at the $386 \mathrm{~m} /$ pixel scale of the base map; topographic relief

120 as expressed in the global stereo digital elevation model (Schenk et al., 2018) was generally not

121 useful for unit definition due to the extreme flatness of the plains. In this work we focus on 13 of

122 the 15 units presented in White et al. (2017), excluding the Impact Crater (ic) and Bright, Pitted

123 Uplands (bpu) units because they are exterior to Sputnik Planitia. Figure 1 - which corresponds

124 to Figure 5 in White et al. (2017) - shows the Sputnik Planitia geological map, and Table 1 
125 describes the diagnostic characteristics and interpretations of these units as presented in White et 126 al. (2017).

127 Cellular plains occupy the core of Sputnik Planitia, with cell boundaries consisting of trough 128 and ridge morphology. The cellular plains are brighter in the central-south area (bcp), and darker 129 in the central-northern part (dcp). The northern margin of Sputnik Planitia shows very low130 albedo plains (tbp), but within which cell boundaries can still be discerned. The western rim of 131 Sputnik Planitia is occupied by chains of randomly oriented blocky mountains (abm) - embayed 132 by inter-block material (ibm) - and by dark-pitted, marginal plains (dmp), and dark, ridged 133 terrain (drt). A few isolated mountain blocks of unit abm are seen within the plains of unit dcp, 134 separated from the main ranges. The spatially largest abm area inside the cellular plains of 135 Sputnik Planitia is Coleda de Dados Colles. To the east and south, Sputnik Planitia shows four 136 types of pitted plains. The lightly pitted plains (lpp) bound the cellular plains along their eastern 137 margin, the sparsely (spp) and deeply pitted plains (dpp) dominate the southernmost region of 138 Sputnik Planitia, and the patchy, pitted, marginal plains (pmp) separate the interior plains from 139 the surrounding highlands along the eastern and southwestern boundaries of Sputnik Planitia. 140 These pitted plains units are concentrated within the eastern and southern regions of Sputnik 141 Planitia, but small expanses of the units are identified on the northern and western edges as well, 142 where they are embayed by the cellular plains and mountain units. Conglomerations of blocks 143 reaching a few $\mathrm{km}$ across are seen within the cellular and pitted plains in the eastern portion of 144 Sputnik Planitia, forming the scattered hills unit (sh).

145 The 13 units that form Sputnik Planitia and its mountain ranges do not commonly concentrate 146 in a single, spatially coherent region, and often occur in several localities across the mapping 147 area that are isolated from each other. To gauge geographical variation in the spectral properties 
148 of these units, we have divided the Sputnik Planitia map into 15 provinces* (Figure 2). These

149 provinces typically display contiguous geomorphological characteristics across their expanse,

150 specifically cellular plains for the "Cellular plains" province, non-cellular plains for the "south

151 pitted plains" and "east pitted plains", "southwest buffer", "west pitted plains" and "north pitted

152 plains" provinces, and mountainous and hilly terrain for the ten "Montes" and "Colles"

153 provinces. This strategy allows us to define the bulk spectral characteristics of each of the 13

154 units across the entire mapping area, and also the spectral characteristics for isolated occurrences

155 of each unit within these separate provinces. This in turn will allow us to assess the degree to

156 which spatially distant occurrences of a particular unit are spectrally similar, and if their spectra

157 do vary with location, then what factors contribute to such variation.

158 The scientific payload onboard NH designed to investigate the spectral characteristics of

159 Pluto's surface is Ralph, a multispectral imager composed by two different instrument sub-

160 systems covering the visible (VIS) and near infrared (NIR) regions of the spectrum with two

161 independent spectrometers. The first component is MVIC, the Multi-spectral Visible Imaging

162 Camera, with its three VIS and NIR channels, blue (400-550 nm), red (540-700 nm), and NIR

163 (780-975 nm), and a narrow $\mathrm{CH}_{4}$ band filter $(860-910 \mathrm{~nm})$. The second instrument sub-system is

164 the Linear Etalon Imaging Spectral Array (LEISA). LEISA has two filter segments, the first

165 covering the $1.25-2.5 \mu \mathrm{m}$ short wave infrared (SWIR) spectral region (spectral power $\lambda / \Delta \lambda=$

$166240)$ to produce spectral maps, the second covering the $2.1-2.25 \mu \mathrm{m}$ range $(\lambda / \Delta \lambda=560)$ to

167 investigate the solid-N $\mathrm{N}_{2}$ absorption feature centered at $2.15 \mu \mathrm{m}$. LEISA scans the $0.9^{\circ} \times 0.9^{\circ} \mathrm{FOV}$

168 in a push broom fashion (Reuter et al., 2009). In this work we used the data from the LEISA

169 SWIR segment only. 
We considered three high spatial resolution (ranging between $\sim 6$ and $\sim 9 \mathrm{~km} /$ pixel) LEISA

171 spectral image cubes to produce a mosaic of Sputnik Planitia with the Integrated Software for

172 Imagers and Spectrometers (ISIS) routines. The image cubes were first projected on a common

173 simple cylindrical map, centered at $180^{\circ} \mathrm{E}$ and $0^{\circ} \mathrm{N}$, with a pixel resolution to $2 \mathrm{~km} / \mathrm{px}$ (smaller

174 than the original resolution of the three LEISA scans). We then converted the I/F radiance (as

175 defined in Hapke, 2012) into a reflectance factor $(R E F F)$ to minimize the effect of solar

176 incidence on the reflected radiance and maximize the absorbing and scattering properties of the

177 surface materials (Schmitt et al., 2017). The REFF is calculated, for each pixel, by dividing the

$178 \mathrm{I} / \mathrm{F}$ radiance by the cosine of the incidence angle (Hapke, 2012), assuming isotropic scattering of

179 the surface (Lambertian photometry). The I/F values have been corrected by the scaling factor in

180 the LEISA radiometric calibration of $0.74 \pm 0.05$ reported by Protopapa et al. 2020 . The three

181 projected cubes were then averaged together to create the final spectral mosaic. LORRI basemap

182 used by White et al. (2017) for the geologic classification, was projected and sampled at the

183 same scale of the LEISA mosaic, so to precisely compare them. We selected regions of interest

184 on the LEISA mosaic corresponding to the Sputnik Planitia geologic units identified in White et

185 al. (2017) by using the ENVI image analysis software.

Table 1. Descriptions and interpretations of 13 geologic units within Sputnik Planitia mapped by White et al. (2017).

\begin{tabular}{lll} 
Unit & Description & Interpretation \\
$\begin{array}{l}\text { Bright, Cellular } \\
\text { plains (bcp) }\end{array}$ & $\begin{array}{l}\text { High albedo plains divided into ovoid } \\
\text { cells by a network of troughs. Cell } \\
\text { interiors typically display a pitted }\end{array}$ & $\begin{array}{l}\text { Plains of } \mathrm{N}_{2}-\mathrm{CO} \text { ice undergoing } \\
\text { continuous resurfacing by solid-state } \\
\text { convection. High albedo is caused by }\end{array}$ \\
\hline
\end{tabular}




\begin{tabular}{|c|c|c|}
\hline & $\begin{array}{l}\text { texture at a scale of a few hundred } \\
\text { meters towards their edges. } \\
\text { Concentrates in central Sputnik } \\
\text { Planitia. }\end{array}$ & $\begin{array}{l}\text { condensation of atmospheric } \mathrm{N}_{2}-\mathrm{CO} \text { onto } \\
\text { the Dark, Cellular plains to form a thin } \\
\text { mantle of pure } \mathrm{N}_{2}-\mathrm{CO} \text { ice. }\end{array}$ \\
\hline $\begin{array}{l}\text { Dark, Cellular } \\
\text { plains (dcp) }\end{array}$ & $\begin{array}{l}\text { Similar to the Bright, Cellular plains, } \\
\text { but with a lower albedo. Concentrates } \\
\text { in northern and western Sputnik } \\
\text { Planitia, but also appears as enclaves } \\
\text { within the Bright, Cellular plains near } \\
\text { the main contact between the two } \\
\text { units. }\end{array}$ & $\begin{array}{l}\text { Unit is deficient in } \mathrm{N}_{2}-\mathrm{CO} \text { relative to the } \\
\text { Bright, Cellular plains and represents the } \\
\text { exposed portion of the convecting } \mathrm{N}_{2}-\mathrm{CO} \\
\text { ice mass, which displays a high content of } \\
\text { entrained tholin accumulated. }\end{array}$ \\
\hline $\begin{array}{l}\text { Dark, Trough- } \\
\text { Bounding Plains } \\
\text { (tbp) }\end{array}$ & $\begin{array}{l}\text { Low albedo plains at the northern } \\
\text { edge of Sputnik Planitia that surround } \\
\text { cell margins, which appear as narrow, } \\
\text { dark lines. Exhibit flow patterns } \\
\text { around obstacles at the margin with } \\
\text { the surrounding uplands. }\end{array}$ & $\begin{array}{l}\mathrm{N}_{2}-\mathrm{CO} \text { ice with an especially high } \\
\text { concentration of entrained tholins at the } \\
\text { edges of the cells and in the bounding } \\
\text { troughs. Flow patterns indicate recent } \\
\text { mobility of the } \mathrm{N}_{2}-\mathrm{CO} \text { ice. }\end{array}$ \\
\hline $\begin{array}{l}\text { Lightly Pitted } \\
\text { Plains (lpp) }\end{array}$ & $\begin{array}{l}\text { High albedo plains displaying a } \\
\text { shallow, densely pitted texture at a } \\
\text { scale of a few hundred meters. }\end{array}$ & $\begin{array}{l}\text { Sublimation of } \mathrm{N}_{2}-\mathrm{CO} \text { ice across the } \\
\text { surface of the plains. }\end{array}$ \\
\hline $\begin{array}{l}\text { Deeply Pitted } \\
\text { Plains (dpp) }\end{array}$ & $\begin{array}{l}\text { High albedo plains featuring dense } \\
\text { concentrations of dark-floored pits } \\
\text { reaching a few km across. Pits can be } \\
\text { single or merge to form doublets and } \\
\text { chains of elongate, aligned pits. Pit } \\
\text { swarms sometimes form wavelike } \\
\text { patterns. }\end{array}$ & $\begin{array}{l}\text { Sublimation pits that may be widening } \\
\text { existing fractures formed by ice shearing } \\
\text { in the } \mathrm{N}_{2}-\mathrm{CO} \text { ice plains. Floors are covered } \\
\text { by dark tholins. Wavelike patterns may } \\
\text { reveal stress fields associated with flow } \\
\text { paths. }\end{array}$ \\
\hline $\begin{array}{l}\text { Sparsely Pitted } \\
\text { Plains (spp) }\end{array}$ & $\begin{array}{l}\text { Smooth, high albedo plains featuring } \\
\text { scattered pits with subdued and } \\
\text { softened relief. }\end{array}$ & $\begin{array}{l}\text { Plains that have been resurfaced by flow of } \\
\mathrm{N}_{2}-\mathrm{CO} \text { ice, and/or blanketing by } \\
\text { atmospheric } \mathrm{N}_{2}-\mathrm{CO} \text { condensation, which } \\
\text { acts to relax/infill pit topography. }\end{array}$ \\
\hline
\end{tabular}

\begin{tabular}{|c|c|c|}
\hline $\begin{array}{l}\text { Patchy, Pitted, } \\
\text { Marginal Plains } \\
\text { (pmp) }\end{array}$ & $\begin{array}{l}\text { High albedo, often-hummocky plains } \\
\text { on the margins of Sputnik Planitia. } \\
\text { Generally, it displays a patchy texture } \\
\text { of close-spaced shallow pits } \\
\text { interspersed with smooth, featureless } \\
\text { zones. Dark flow lines are often seen } \\
\text { where the unit occurs along the border } \\
\text { with the Bright, Pitted Uplands. }\end{array}$ & $\begin{array}{l}\text { Shallow } \mathrm{N}_{2}-\mathrm{CO} \text { ice draped over underlying } \\
\text { topography, which may contribute to the } \\
\text { heterogeneous texture of the unit. Glacial } \\
\text { flow of the unit is occurring from valleys } \\
\text { in the Bright, Pitted Uplands to the open } \\
\text { plains of Sputnik Planitia. }\end{array}$ \\
\hline $\begin{array}{l}\text { Dark-Pitted, } \\
\text { Marginal Plains } \\
(\text { dmp) }\end{array}$ & $\begin{array}{l}\text { High albedo plains displaying chains } \\
\text { of elongate, dark-floored pits } \\
\text { interspersed with smooth, lightly } \\
\text { pitted zones. Unit occurs on the } \\
\text { western edge of Sputnik Planitia. }\end{array}$ & $\begin{array}{l}\text { Shallow } \mathrm{N}_{2}-\mathrm{CO} \text { ice draped over underlying } \\
\text { topography. The ice may be residual and } \\
\text { relatively immobile compared to that of } \\
\text { the Patchy, Pitted, Marginal Plains. Dark } \\
\text { pit floors represent Cthulhu Macula, which } \\
\text { this unit overlies. }\end{array}$ \\
\hline $\begin{array}{l}\text { Chaotic, } \\
\text { Angular, Blocky } \\
\text { Mountains (abm) }\end{array}$ & $\begin{array}{l}\text { Discontinuous chain of mountain } \\
\text { ranges, consisting of angular blocks } \\
\text { with random orientations. Ranges }\end{array}$ & $\begin{array}{l}\text { Fragments of the } \mathrm{H}_{2} \mathrm{O} \text { ice crust that have } \\
\text { been detached by tectonism and intruded, } \\
\text { transported, and rotated by glacial } \mathrm{N}_{2}-\mathrm{CO}\end{array}$ \\
\hline
\end{tabular}




\begin{tabular}{|c|c|c|}
\hline & $\begin{array}{l}\text { extend along the western margin of } \\
\text { Sputnik Planitia, with a few isolated } \\
\text { blocks occurring within its interior. }\end{array}$ & $\begin{array}{l}\text { ice. The mountain blocks are now } \\
\text { grounded within shallow } \mathrm{N}_{2} \text {-CO ice of } \\
\text { Sputnik Planitia. }\end{array}$ \\
\hline $\begin{array}{l}\text { Chaotic, Inter- } \\
\text { Block Material } \\
\text { (ibm) }\end{array}$ & $\begin{array}{l}\text { Chaotically oriented, close-packed } \\
\text { blocks, reaching several km across, } \\
\text { that are interstitial to and embay the } \\
\text { Chaotic, Angular, Blocky Mountains. }\end{array}$ & $\begin{array}{l}\text { Like the Chaotic, Angular, Blocky } \\
\text { Mountains, but finer texture may indicate } \\
\text { breaking up of mountain blocks into } \\
\text { smaller fragments and/or size filtering } \\
\text { thereafter. }\end{array}$ \\
\hline $\begin{array}{l}\text { Scattered Hills } \\
\text { (sh) }\end{array}$ & $\begin{array}{l}\text { Hills scattered across east Sputnik } \\
\text { Planitia that reach a few km across } \\
\text { and tend to collect into densely } \\
\text { packed clusters. Chains of aligned } \\
\text { hills occur proximal to the Bright, } \\
\text { Pitted Uplands. In the Bright, Cellular } \\
\text { plains, the hills are always coincident } \\
\text { with the cell boundaries. }\end{array}$ & $\begin{array}{l}\mathrm{CH}_{4} \text { ice fragments eroded from the Bright, } \\
\text { Pitted Uplands that have rafted into } \\
\text { Sputnik Planitia on valley glaciers of } \\
\text { denser } \mathrm{N}_{2} \text {-CO ice. In the Bright, Cellular } \\
\text { plains, convective motions corral them } \\
\text { into clusters at cell boundaries. }\end{array}$ \\
\hline $\begin{array}{l}\text { Dark, Ridged } \\
\text { Terrain (drt) }\end{array}$ & $\begin{array}{l}\text { Situated between the Chaotic, } \\
\text { Angular, Blocky Mountains of } \\
\text { western Sputnik Planitia and uplands } \\
\text { to the west. Aligned ridges are } \\
\text { oriented parallel to the edge of } \\
\text { Sputnik Planitia, and display } \\
\text { wavelengths from several hundred } \\
\text { meters to a few km. }\end{array}$ & $\begin{array}{l}\text { Possibly Chaotic, Inter-Block Material } \\
\text { covered by dark mantling material. } \\
\text { Compression of this unit by pressure } \\
\text { exerted from westward motion of the } \\
\text { Chaotic, Angular, Blocky Mountains may } \\
\text { produce the ridges. }\end{array}$ \\
\hline $\begin{array}{l}\text { Bright, Chaotic } \\
\text { Terrain (bct) }\end{array}$ & $\begin{array}{l}\text { High albedo plains interspersed with } \\
\text { darker blocks reaching several km } \\
\text { across, on the northwest margin of } \\
\text { Sputnik Planitia. E-W oriented } \\
\text { troughs and scarps cross the unit. } \\
\text { Parts of the unit are depressed by } \\
\text { several hundred meters below the } \\
\text { bordering Chaotic Inter-Block } \\
\text { Material. }\end{array}$ & $\begin{array}{l}\text { Unit represents uplands material that is } \\
\text { experiencing extensional tectonism and } \\
\text { subsidence, and possible intrusion by } \mathrm{N}_{2-} \\
\mathrm{CO} \text { ice of Sputnik Planitia. High albedo of } \\
\text { the plains indicates a surface covering of } \\
\mathrm{N}_{2} \text {-CO ice, possibly 'overflowed' from } \\
\text { Sputnik Planitia. Unit may represent an } \\
\text { incipient stage of formation of the Chaotic, } \\
\text { Angular, Blocky Mountains. }\end{array}$ \\
\hline
\end{tabular}


$203(1.25-2.5 \mu \mathrm{m})$, the spectrum of Sputnik Planitia is dominated, on average, by $\mathrm{CH}_{4}, \mathrm{~N}_{2}$, and $\mathrm{CO}$

204 ice absorptions (e.g. Grundy et al., 2016). Some regions also show the presence of $\mathrm{H}_{2} \mathrm{O}$ ice. $\mathrm{CH}_{4}$

205 has four series of broad absorptions in the region between 1.30-1.43 $\mu \mathrm{m}$ (Range 1), 1.59-1.83

$206 \mu \mathrm{m}$ (Range 2), 1.90-2.00 $\mu \mathrm{m}$ (Range 3), and 2.09-2.48 $\mu \mathrm{m}$ (Range 4), and a single band centered

207 at $1.48 \mu \mathrm{m}$ (Schmitt et al., 2017). $\mathrm{CO}$ and $\mathrm{N}_{2}$ absorption bands are centered at $1.58 \mu \mathrm{m}$ and 2.15

$208 \mu \mathrm{m}$, respectively.

209

210

Figure 3: Average spectra of the 13 geologic units identified by White et al. (2017). The boxes indicate the location of the main

211 absorption bands of $\mathrm{CH}_{4}, \mathrm{CO}$ and $\mathrm{N}_{2}$.

\subsection{Absorption bands analysis}

214 We calculated the integrated band depth of the main $\mathrm{CH}_{4}, \mathrm{~N}_{2}, \mathrm{CO}$, and $\mathrm{H}_{2} \mathrm{O}$ ice absorptions in

215 LEISA spectra by following the method of Schmitt et al. (2017):

$$
B D_{\lambda_{1} \lambda_{2}}=1-\frac{\int_{\lambda_{1}}^{\lambda_{2}} \operatorname{REFF}(\lambda) d \lambda}{\int_{\lambda_{1}}^{\lambda_{2}} \operatorname{Cont}(\lambda) d \lambda}
$$

$217 \lambda_{1}$ and $\lambda_{2}$ are the positions of the right and left shoulders of the absorption bands, listed in

218 Table 2. To limit the effect of random noise, we calculated the shoulder's continuum value by

219 averaging the reflectance of five points around $\lambda_{1}$ and $\lambda_{2} \operatorname{REFF}(\lambda)$ is the reflectance at the bottom

220 of the band, integrated over the wavelength range between $\lambda_{1}$ and $\lambda_{2}$, and $\operatorname{Cont}(\lambda)$ is the

221 continuum, calculated as a best fit line between the two shoulders.

Table 2: List of absorption bands, with the range of wavelengths used to evaluate the positions of the right and left shoulders for each of them.

\begin{tabular}{clcl} 
Ice & Nominal minimum position & Right shoulder & Left shoulder \\
$\mathrm{CH}_{4}$ & $\begin{array}{l}\text { Range } 1 \\
1.30-1.43 \mu \mathrm{m}\end{array}$ & $1.2929-1.3116 \mu \mathrm{m}$ & $1.4205-1.4417 \mu \mathrm{m}$ \\
\hline
\end{tabular}




\begin{tabular}{llll} 
& & & \\
& Range 2 & \\
& $1.59-1.83 \mu \mathrm{m}$ \\
& Range 3 \\
& $1.90-2.00 \mu \mathrm{m}$ & $1.5777-1.6004 \mu \mathrm{m}$ & $1.8202-1.8466 \mu \mathrm{m}$ \\
& Range 4 \\
& $2.09-2.48 \mu \mathrm{m}$ \\
& $1.48 \mu \mathrm{m}$ & $1.8871-1.9147 \mu \mathrm{m}$ & $1.9857-2.0149 \mu \mathrm{m}$ \\
& $1.58 \mu \mathrm{m}$ & $2.0747-2.1053 \mu \mathrm{m}$ & $2.4723-2.4892 \mu \mathrm{m}$ \\
& $2.15 \mu \mathrm{m}$ & $1.4681-1.4734 \mu \mathrm{m}$ & $1.4895-1.5003 \mu \mathrm{m}$ \\
\hline $\mathrm{CO}$ & $2.02 \mu \mathrm{m}$ & $1.5552-1.5777 \mu \mathrm{m}$ & $1.5777-1.6004 \mu \mathrm{m}$ \\
\hline $\mathrm{N}_{2}$ & & $2.1207-2.1519 \mu \mathrm{m}$ & $2.1441-2.1756 \mu \mathrm{m}$ \\
\hline $\mathrm{H}_{2} \mathrm{O}$ & $1.8371-1.8638 \mu \mathrm{m}$ & $2.1647-2.1965 \mu \mathrm{m}$ \\
\hline \hline
\end{tabular}

\section{2 $\mathrm{CH}_{4}$ band shifts}

226 Solid nitrogen $\left(\mathrm{N}_{2}\right)$ can appear in two different, temperature-dependent states: cubic, or $\alpha$-,

227 and hexagonal, or $\beta$ - (e.g., Grundy et al., 1993). $\mathrm{N}_{2}$ absorption on Pluto is consistent with the $\beta$ -

228 phase, stable at T > 35.6 K (Quirico \& Schmitt, 1997; Grundy et al., 2013).

$229 \mathrm{CH}_{4}$ and $\mathrm{N}_{2}$ form complex mixtures on the surface of Pluto since these two ices attract each

230 other at the molecular level. Prokhvatilov and Yantsevich (1983) demonstrated that at Pluto's

231 average surface temperature $(40 \mathrm{~K}), \mathrm{CH}_{4}$ and $\mathrm{N}_{2}$ ice are partly soluble and can form two mixing

232 phases: a $\mathrm{CH}_{4}$-rich component that is $\mathrm{CH}_{4}$ saturated with $\mathrm{N}_{2}\left(\mathrm{CH}_{4}: \mathrm{N}_{2}\right)$, and a $\mathrm{N}_{2}$-rich component

233 that is $\mathrm{N}_{2}$ saturated with $\mathrm{CH}_{4}\left(\mathrm{~N}_{2}: \mathrm{CH}_{4}\right)$. When a small concentration of $\mathrm{CH}_{4}$ is dissolved within

$234 \mathrm{~N}_{2}, \mathrm{CH}_{4}$ absorption bands show a shift towards shorter wavelengths (Schmitt \& Quirico, 1992;

235 Quirico \& Schmitt, 1997), with the shift decreasing with increasing amount of $\mathrm{CH}_{4}$ dissolved in

236 the $\mathrm{N}_{2}$ (Protopapa et al., 2015). This means that smaller values of the observed $\mathrm{CH}_{4}$ band shift

237 correspond to higher fraction of pure $\mathrm{CH}_{4}$ ice.

238 To quantify the amount of $\mathrm{CH}_{4}$ dissolved in the $\mathrm{N}_{2}$ matrix for each geologic unit across

239 Sputnik Planitia, we used the following method to estimate the average shift of the $\mathrm{CH}_{4}$

240 absorptions. We take a pure $\mathrm{CH}_{4}$ ice spectrum model, with a grain size of $0.5 \mathrm{~mm}$, as reference. 
241 The following procedure is applied to both LEISA and the reference pure $\mathrm{CH}_{4}$ spectrum. We

242 consider the left and right shoulders of each $\mathrm{CH}_{4}$ absorption band listed in Table 3 (for the

243 triplets, we considered each local minimum), and we calculated a straight-line continuum

244 between the two shoulders. For each absorption feature (and local minimum), we divided the

245 spectrum by the straight-line continuum. The continuum-removed bands are fit with a cubic

246 spline with an increment of 100 steps, and we assumed the absolute minimum of the spline curve

247 as the band's minimum position. The shift for each minimum is defined as the difference

248 between the pure $\mathrm{CH}_{4}$ ice and the LEISA spline estimated minima. We have, at the end of this

249 process, eight different band shifts values. From our calculations, and from Schmitt et al. (2017),

250 each individual $\mathrm{CH}_{4}$ absorption shows a different band's minimum shift. We calculated the

251 average $\mathrm{CH}_{4}$ band shift parameter by averaging the eight shift values together. The shift towards

252 shorter wavelengths (increasing $\mathrm{N}_{2}$-rich ice) is indicated by an increasing positive value of the

253 shift index.

254

Table 3: List of $\mathrm{CH}_{4}$ absorption bands in the wavelength range covered by LEISA, with the range of wavelengths used to evaluate the positions of the right and left shoulders for each of them.

\begin{tabular}{llll}
$\mathrm{CH}_{4}$ range & Nominal minimum position & Right shoulder & Left shoulder \\
& $1.33 \mu \mathrm{m}$ & $1.3093 \mu \mathrm{m}$ & $1.3453 \mu \mathrm{m}$ \\
$\begin{array}{l}\text { Range } 1-\text { triplet } \\
1.30-1.43 \mu \mathrm{m}\end{array}$ & $1.36 \mu \mathrm{m}$ & $1.3453 \mu \mathrm{m}$ & $1.3827 \mu \mathrm{m}$ \\
& $1.40 \mu \mathrm{m}$ & $1.3827 \mu \mathrm{m}$ & $1.4200 \mu \mathrm{m}$ \\
\hline Single band & $1.48 \mu \mathrm{m}$ & $1.4681 \mu \mathrm{m}$ & $1.4949 \mu \mathrm{m}$ \\
\hline $\begin{array}{l}\text { Range } 2-\text { triplet } \\
1.59-1.83 \mu \mathrm{m}\end{array}$ & $1.66 \mu \mathrm{m}$ & $1.6271 \mu \mathrm{m}$ & $1.6948 \mu \mathrm{m}$ \\
\hline $\begin{array}{l}\text { Range } 3-\text { broad band } \\
1.90-2.00 \mu \mathrm{m}\end{array}$ & $1.72 \mu \mathrm{m}$ & $1.6948 \mu \mathrm{m}$ & $1.7419 \mu \mathrm{m}$ \\
\hline \hline
\end{tabular}




\subsection{Radiative transfer model}

The spectra of the geologic units inside each province have been modeled by using a

259 modified version of the Hapke theory (i.e. Hapke et al., 1993; 2012). The Hapke parameters used

260 here are from Verbiscer et al. (2019): the Henyey-Greenstein single particle phase function

261 asymmetry parameter $\mathrm{g}=-0.36$, and the amplitude and width of the opposition surge due to

262 shadow hiding, $\mathrm{B}_{0}=0.307$ and $\mathrm{h}=0.206$ respectively. Coherent backscatter was not considered in

263 the model. For a full and complete description of the Hapke methodology we employed here, we

264 refer to Cook et al., (2019).

265 The models consider several endmember components, combined into two spatially

266 separate units by considering an areal mixture. In Tables 4 and 5, the two units are labeled with

267 the letters A and B. Each unit can have one or more endmembers. When more than one

268 endmember is considered, they are intimately mixed together.

269 As proven by previous work (e.g. Protopapa et al., 2017; Schmitt et al., 2017), $\mathrm{CH}_{4}$ is

270 ubiquitous within Sputnik Planitia. It can either be diluted within $\mathrm{N}_{2}$-rich ice, or it can be a pure

271 endmember (Douté et al., 1999; Young et al., 2018). We considered optical constants of $\mathrm{CH}_{4}$ ice

272 diluted in $\mathrm{N}_{2}$ ice from Protopapa et al. (2015), and pure $\mathrm{CH}_{4}$ ice optical constants from Grundy et

273 al. (2002). Pure $\mathrm{CH}_{4}$ is used here as a substitute for the $\mathrm{CH}_{4}$-rich component $\left(\mathrm{CH}_{4}: \mathrm{N}_{2}\right)$, since

274 previous studies have shown that the wavelength shifts are very small in that phase (Protopapa et

275 al., 2017). $\mathrm{N}_{2}$ can also appear on the surface as pure ice. We used optical constants from Grundy

276 et al. (1993).

277 The spectra of several geologic units have a marked negative spectral slope in the wavelength

278 range covered by LEISA. After several attempts to model the negative spectral slope with a wide

279 range of components, we concluded that the optical constants of the clay mineral kaolinite fit this

* Some feature names mentioned in this paper are now formalized while others are informal 
280 spectral feature the best. Figure 4 shows the spectrum of unit drt in Baret montes - the region 281 with the strongest negative spectral slope - compared with seven Hapke models, calculated by

282 considering the optical constants of seven different materials selected to account for the observed

283 negative spectral slope: amorphous carbon, kaolinite, montmorillonite, Pluto tholin, serpentine,

284 Titan tholin, and Triton tholin. Kaolinite returned the best result.

290 scatter greater at shorter wavelengths. For this paper, however, we assume that the negative

291 spectral slope is due to a separate endmember and adopt kaolinite as representative of the

292 negative spectral slope endmember (NSS endmember).

293 A negative spectral slope can be explained also by physical properties of the surface material, 294 such as a surface coating of ultra-fine grains (Stephan et al., 2017, Protopapa et al. 2020). We do 295 not consider ultra-fine grains in this work, as it is outside the scope of this work.

296 The NSS endmember is presumed to be a relatively refractory non-ice macromolecular 297 carbon-rich organic material formed by energetic processing of Pluto's atmosphere of $\mathrm{CH}_{4}, \mathrm{~N}_{2}$, $298 \mathrm{CO}$, and other minor gaseous components, as well as the solid forms of these molecules in the 299 surface ices. Both ultraviolet radiation and charged particles readily transform these simple 300 molecules into disordered macromolecular complexes generally termed tholins. Tholins exhibit 301 a range of yellow-to-red color in the visible spectral region and are thought to account for the red 302 colors of numerous Solar System bodies (e.g., Cruikshank et al. 2005). Materese et al. (2014, 303 2015) determined the compositions and structures of tholins produced by UV photolysis and 304 low-voltage electron radiolysis of an ice mixture of $\mathrm{N}_{2}, \mathrm{CH}_{4}$, and $\mathrm{CO}$ (100:1:1) relevant to 
305 Pluto's surface. The radiolytic produced tholin was further analyzed by Cruikshank et al. (2016),

306 demonstrating its negative spectral slope at $\lambda>1.1 \mu \mathrm{m}$.

307 Tholins synthesized in gas phase experiments appropriate for modeling the spectra of the 308 atmospheres of Titan or Triton have positive rather than a negative spectral slope (Khare et al.,

$3091984 ; 1993 ; 1994)$, and do not introduce a sufficiently negative spectral slope in models of

310 LEISA spectra of Pluto's surface units (Figure 4) unless accounting for fine grains. Indeed,

311 Protopapa et al. 2020 reproduces successfully Pluto's MVIC+LEISA observations using Titan

312 tholin like material with ultra-fine grains. As we explained before, we will not explore the

313 contribution of fine grain in this paper. At the same time, there is very little published

314 information on the optical constants for tholins synthesized in ices. Cruikshank et al. (2016)

315 published a reflectance spectrum of the tholin produced by electron radiolysis of the $\mathrm{CH}_{4}: \mathrm{N}_{2}: \mathrm{CO}$

316 ice mixture in the experiments of Materese et al. (2015) considered to be relevant to Pluto's

317 surface. Optical constants were derived from that reflectance spectrum by assuming a grain size

318 of $10 \mu \mathrm{m}$ (Protopapa et al. 2021). While the derived optical constants for that Plutonian tholin

319 show a subtle negative spectral slope trend, it is insufficient to account for the negative spectral

320 slope found in LEISA spectra (Cook et al., 2019). To test the optical constants of Plutonian

321 tholins, and to compare them to the results obtained with the NSS endmember, we selected four

322 spectra to be modeled with the inclusion of the Plutonian tholin. The results are discussed in

323 detail in Section 4.4. Three of these spectra were taken from unit drt within the Baret, Hillary,

324 and Al-Idrisi Montes provinces, located on the western rim of Sputnik Planitia, as they show

325 particularly negative spectral slope spectra. The last spectrum was taken from unit sh in the

326 Columbia Colles province. This was selected because, even though this unit is located on the

327 eastern perimeter of Sputnik Planitia and was classified as the "scattered hills" unit by White et 
al. (2017), it does show spectral and geomorphological characteristics that are more similar to

329 those of unit drt than to those of other occurrences of unit sh (see Section 5 for further details).

330 The mountain ranges that line the western perimeter of Sputnik Planitia are composed of $\mathrm{H}_{2} \mathrm{O}$

331 ice (Grundy et al., 2016; Protopapa et al., 2017; Schmitt et al., 2017; Cook et al., 2019). We

332 consider both crystalline and amorphous $\mathrm{H}_{2} \mathrm{O}$ ice, with optical constants from Grundy \& Schmitt

333 (1998) and Mastrapa et al. (2008) respectively.

334 The optical constants for $\mathrm{CH}_{4}: \mathrm{N}_{2}$ mixtures, pure $\beta-\mathrm{N}_{2}$ and $\mathrm{H}_{2} \mathrm{O}$-ices are known at specific

335 temperature values. The optical constants at intermediate values of temperature are calculated in

336 our models by linearly interpolating the optical constants at measured temperatures, and by

337 searching for the temperature which minimizes the $\chi^{2}$. However, the changes in $\chi^{2}$ due to

338 temperature variation are typically small and can confidently constrain the temperature only

339 between 40 and $60 \mathrm{~K}$. The temperatures reported in this paper are the best-fit values only.

340 The endmembers used for each geologic unit in the 15 provinces are listed in Table 4 . The

341 first areal mixture (unit 0 in Table 4) includes higher-volatility materials, namely $\mathrm{CH}_{4}$ (pure or

342 diluted in $\mathrm{N}_{2}$-rich ice) and pure $\beta-\mathrm{N}_{2}$. The second unit (unit 1) includes lower-volatility materials,

343 namely crystalline or amorphous $\mathrm{H}_{2} \mathrm{O}$ ice, and NSS endmember.

344 Figure 5 shows the average LEISA spectra of the geologic units for each province,

345 superimposed on the corresponding model. It must be noted that the model never considers the

346 CO $1.58 \mu \mathrm{m}$ spectral region because of lack of reliable optical constants (Protopapa et al., 2017).

347 This band corresponds to the (3-0) overtone, which is weaker than the (2-0) band centered at

$348 \quad 2.35 \mu \mathrm{m}$ (seen in ground-based, disk-integrated observations). Even though reliable optical

349 constants are available for the $\mathrm{CO}$ spectral region around $2.35 \mu \mathrm{m}$, it lies in a gap in our

350 knowledge of the optical constants for $\mathrm{CH}_{4}: \mathrm{N}_{2}$. Furthermore, the $\mathrm{CH}_{4}$ bands appear saturated in 
351 the data, and any attempt to accommodate for the diluted $\mathrm{CH}_{4}$ optical constants without proper

352 understanding of the bands would likely yield large uncertainties in our fit for the CO band at

$3532.35 \mu \mathrm{m}$.

354 To understand the areal abundance of the endmembers in each unit, we calculated the areal

355 mass fraction as the product of the endmember's mass fraction with its corresponding fractional

356 area. This parameter represents the surface percentage of each geologic unit covered by each

357 endmember, and it will be used in the compositional maps to indicate the abundance of each

358 endmember in the geologic units (see section 4 for further details).

Table 4: Parameters used to model the LEISA spectra with a modified version of the Hapke theory (i.e. Hapke 1993, 2012).

\begin{tabular}{ccccccccc} 
& & & & \multicolumn{2}{c}{ Areal } \\
Province $^{*}$ & $\mathrm{~T}(\mathrm{~K})$ & fraction & Component & Unit & Mass & Grain & Fractional mass \\
& & $(\%)$ & & & fraction & $(\mathrm{mm})$ & Area & fraction \\
& & & & & $(\%)$
\end{tabular}

ABM

\begin{tabular}{|c|c|c|c|c|c|c|c|c|}
\hline \multirow{3}{*}{ Baret Montes } & \multirow{3}{*}{44} & \multirow{3}{*}{1.20} & $\mathrm{~N}_{2}: \mathrm{CH}_{4}$ & A & 1.000 & 297.9 & 0.214 & 21.4 \\
\hline & & & NSS endmember & \multirow{2}{*}{ B } & 0.467 & 0.004 & \multirow{2}{*}{0.786} & 36.7 \\
\hline & & & Crystalline $\mathrm{H}_{2} \mathrm{O}$ & & 0.533 & 0.20 & & 41.9 \\
\hline \multirow{4}{*}{$\begin{array}{l}\text { Cellular } \\
\text { plains }\end{array}$} & \multirow{4}{*}{45} & \multirow{4}{*}{1.80} & Pure $\mathrm{CH}_{4}$ & \multirow{2}{*}{ A } & 0.003 & 0.18 & \multirow{2}{*}{0.664} & 0.2 \\
\hline & & & $\mathrm{N}_{2}: \mathrm{CH}_{4}$ & & 0.997 & 70.0 & & 66.2 \\
\hline & & & Crystalline $\mathrm{H}_{2} \mathrm{O}$ & \multirow{2}{*}{$\mathrm{B}$} & 0.871 & 5.66 & \multirow{2}{*}{0.336} & 29.3 \\
\hline & & & Amorphous $\mathrm{H}_{2} \mathrm{O}$ & & 0.129 & 0.03 & & 4.3 \\
\hline \multirow{4}{*}{$\begin{array}{l}\text { Hillary } \\
\text { Montes }\end{array}$} & \multirow{4}{*}{43} & \multirow{4}{*}{0.25} & $\mathrm{~N}_{2}: \mathrm{CH}_{4}$ & \multirow{2}{*}{ A } & 0.007 & 86.0 & \multirow{2}{*}{0.348} & 0.2 \\
\hline & & & $\beta-\mathrm{N}_{2}$ & & 0.993 & $120,453.0$ & & 34.5 \\
\hline & & & NSS endmember & \multirow{2}{*}{$\mathrm{B}$} & 0.426 & 0.01 & \multirow{2}{*}{0.652} & 27.8 \\
\hline & & & Crystalline $\mathrm{H}_{2} \mathrm{O}$ & & 0.574 & 0.331 & & 37.5 \\
\hline \multirow{3}{*}{$\begin{array}{c}\text { Southwest } \\
\text { buffer }\end{array}$} & \multirow{3}{*}{48} & \multirow{3}{*}{1.00} & Pure $\mathrm{CH}_{4}$ & \multirow{2}{*}{ A } & 0.005 & 0.62 & \multirow{2}{*}{0.817} & 0.4 \\
\hline & & & $\mathrm{N}_{2}: \mathrm{CH}_{4}$ & & 0.995 & 595.7 & & 81.3 \\
\hline & & & Crystalline $\mathrm{H}_{2} \mathrm{O}$ & B & 1.0 & 0.01 & 0.183 & 18.3 \\
\hline \multirow{3}{*}{$\begin{array}{l}\text { Tenzing } \\
\text { Montes }\end{array}$} & \multirow{3}{*}{50} & \multirow{3}{*}{0.05} & Pure $\mathrm{CH}_{4}$ & & 0.015 & 1.68 & \multirow{2}{*}{0.907} & 1.4 \\
\hline & & & $\mathrm{N}_{2}: \mathrm{CH}_{4}$ & A & 0.985 & 58.4 & & 89.3 \\
\hline & & & Crystalline $\mathrm{H}_{2} \mathrm{O}$ & $\mathrm{B}$ & 1.0 & 0.30 & 0.093 & 9.3 \\
\hline \multirow{3}{*}{$\begin{array}{l}\text { Zheng He } \\
\text { Montes }\end{array}$} & \multirow{3}{*}{55} & \multirow{3}{*}{2.90} & $\mathrm{~N}_{2}: \mathrm{CH}_{4}$ & $\mathrm{~A}$ & 1.0 & 29.63 & 0.333 & 33.3 \\
\hline & & & Crystalline $\mathrm{H}_{2} \mathrm{O}$ & $\mathrm{P}$ & 0.376 & $4.8 \mathrm{E}-03$ & \multirow{2}{*}{0.667} & 25.1 \\
\hline & & & NSS endmember & D & 0.624 & 0.43 & & 41.6 \\
\hline \multirow{2}{*}{$\begin{array}{l}\text { Al-Idrisi } \\
\text { Montes }\end{array}$} & \multirow{2}{*}{44} & 070 & $\mathrm{~N}_{2}: \mathrm{CH}_{4}$ & A & 1.0 & 120.3 & 0.646 & 64.6 \\
\hline & & 0.10 & Crystalline $\mathrm{H}_{2} \mathrm{O}$ & $\mathrm{B}$ & 1.0 & 0.01 & 0.354 & 35.4 \\
\hline
\end{tabular}

BCP 


\begin{tabular}{|c|c|c|c|c|c|c|c|c|}
\hline $\begin{array}{c}\text { Cellular } \\
\text { plains } \\
\end{array}$ & 46 & 0.60 & $\begin{array}{c}\text { Pure } \mathrm{CH}_{4} \\
\mathrm{~N}_{2}: \mathrm{CH}_{4} \\
\end{array}$ & A & $\begin{array}{c}2.44 \cdot 10^{-5} \\
1.00 \\
\end{array}$ & $\begin{array}{l}0.004 \\
155.1 \\
\end{array}$ & 1.0 & $\begin{array}{c}0.002 \\
99.998 \\
\end{array}$ \\
\hline \multicolumn{9}{|c|}{ BCT } \\
\hline \multirow{2}{*}{$\begin{array}{l}\text { Al-Idrisi } \\
\text { Montes }\end{array}$} & \multirow{2}{*}{44} & \multirow{2}{*}{0.80} & $\begin{array}{c}\mathrm{N}_{2}: \mathrm{CH}_{4} \\
\beta-\mathrm{N}_{2}\end{array}$ & $\mathrm{~A}$ & $\begin{array}{l}0.095 \\
0.905\end{array}$ & $\begin{array}{l}19.719 \\
336.1\end{array}$ & 0.605 & $\begin{array}{c}5.7 \\
54.8\end{array}$ \\
\hline & & & $\begin{array}{l}\text { NSS endmember } \\
\text { Crystalline } \mathrm{H}_{2} \mathrm{O}\end{array}$ & B & $\begin{array}{l}0.353 \\
0.647 \\
\end{array}$ & $\begin{array}{l}0.004 \\
0.153 \\
\end{array}$ & 0.395 & $\begin{array}{l}14.0 \\
25.5 \\
\end{array}$ \\
\hline \multicolumn{9}{|c|}{ DCP } \\
\hline \multirow[t]{2}{*}{$\begin{array}{l}\text { Cellular } \\
\text { plains }\end{array}$} & \multirow[t]{2}{*}{44} & \multirow[t]{2}{*}{1.70} & $\begin{array}{c}\text { Pure } \mathrm{CH}_{4} \\
\mathrm{~N}_{2}: \mathrm{CH}_{4} \\
\beta-\mathrm{N}_{2}\end{array}$ & A & $\begin{array}{c}9.38 \cdot 10^{-5} \\
0.07 \\
0.93\end{array}$ & $\begin{array}{l}0.33 \\
26.0\end{array}$ & 0.731 & $\begin{array}{c}6.86 \cdot 10^{-3} \\
4.9 \\
68.2 \\
\end{array}$ \\
\hline & & & NSS endmember & B & 1.0 & 0.01 & 0.269 & 26.9 \\
\hline \multirow{2}{*}{$\begin{array}{l}\text { Al-Idrisi } \\
\text { Montes }\end{array}$} & \multirow{2}{*}{44} & \multirow{2}{*}{2.30} & $\begin{array}{c}\mathrm{N} 2: \mathrm{CH} 4 \\
\beta-\mathrm{N}_{2}\end{array}$ & A & $\begin{array}{l}0.033 \\
0.967\end{array}$ & $\begin{array}{l}9.208 \\
512.3\end{array}$ & 0.557 & $\begin{array}{c}1.9 \\
53.9\end{array}$ \\
\hline & & & $\begin{array}{l}\text { NSS endmember } \\
\text { Crystalline } \mathrm{H}_{2} \mathrm{O} \\
\end{array}$ & B & $\begin{array}{l}0.467 \\
0.533 \\
\end{array}$ & $\begin{array}{l}0.006 \\
0.071 \\
\end{array}$ & 0.443 & $\begin{array}{l}20.7 \\
23.6 \\
\end{array}$ \\
\hline \multicolumn{9}{|c|}{ DMP } \\
\hline \multirow[t]{2}{*}{$\begin{array}{l}\text { Baret } \\
\text { Montes }\end{array}$} & \multirow[t]{2}{*}{47} & \multirow[t]{2}{*}{2.20} & $\begin{array}{c}\text { Pure } \mathrm{CH}_{4} \\
\mathrm{~N}_{2}: \mathrm{CH}_{4} \\
\beta-\mathrm{N}_{2}\end{array}$ & A & $\begin{array}{l}0.001 \\
0.114 \\
0.885 \\
\end{array}$ & $\begin{array}{c}0.332 \\
44.9 \\
1597.9 \\
\end{array}$ & 0.611 & \begin{tabular}{c|}
0.1 \\
7.0 \\
54.1 \\
\end{tabular} \\
\hline & & & NSS endmember & B & 1.000 & 0.007 & 0.389 & 38.9 \\
\hline \multirow{2}{*}{$\begin{array}{l}\text { Hillary } \\
\text { Montes } \\
\end{array}$} & \multirow{2}{*}{48} & \multirow{2}{*}{1.2} & $\mathrm{~N}_{2}: \mathrm{CH}_{4}$ & $\mathrm{~A}$ & 1.000 & 88.000 & 0.614 & 61.4 \\
\hline & & & NSS endmember & $\mathrm{B}$ & 1.000 & 0.006 & 0.386 & 38.6 \\
\hline \multicolumn{9}{|c|}{ DPP } \\
\hline $\begin{array}{l}\text { Hillary } \\
\text { Montes }\end{array}$ & 48 & 0.60 & $\begin{array}{c}\text { Pure } \mathrm{CH}_{4} \\
\mathrm{~N}_{2}: \mathrm{CH}_{4}\end{array}$ & A & $\begin{array}{l}0.001 \\
0.999\end{array}$ & $\begin{array}{l}0.109 \\
662.8\end{array}$ & 1.0 & $\begin{array}{c}0.1 \\
99.9\end{array}$ \\
\hline $\begin{array}{c}\text { North pitted } \\
\text { plains }\end{array}$ & 45 & 0.45 & $\begin{array}{c}\text { Pure } \mathrm{CH}_{4} \\
\mathrm{~N}_{2}: \mathrm{CH}_{4} \\
\end{array}$ & A & $\begin{array}{c}0.0002 \\
0.999 \\
\end{array}$ & $\begin{array}{l}0.021 \\
224.6 \\
\end{array}$ & 1.0 & \begin{tabular}{|c|}
0.02 \\
99.98 \\
\end{tabular} \\
\hline $\begin{array}{c}\text { Cellular } \\
\text { plains }\end{array}$ & 47 & 0.66 & $\begin{array}{c}\text { Pure } \mathrm{CH}_{4} \\
\mathrm{~N}_{2}: \mathrm{CH}_{4} \\
\end{array}$ & A & $\begin{array}{l}0.001 \\
0.999 \\
\end{array}$ & $\begin{array}{l}0.087 \\
288.3 \\
\end{array}$ & 1.0 & $\begin{array}{c}0.1 \\
99.9 \\
\end{array}$ \\
\hline \multirow{2}{*}{$\begin{array}{l}\text { East pitted } \\
\text { plains }\end{array}$} & \multirow[t]{2}{*}{48} & \multirow[t]{2}{*}{4.50} & $\begin{array}{c}\mathrm{N}_{2}: \mathrm{CH}_{4} \\
\beta-\mathrm{N}_{2}\end{array}$ & A & $\begin{array}{l}0.020 \\
0.980\end{array}$ & $\begin{array}{c}12.7 \\
1868.6\end{array}$ & 0.712 & $\begin{array}{l}1.4 \\
69.8\end{array}$ \\
\hline & & & NSS endmember & $\mathrm{B}$ & 1.000 & 0.022 & 0.288 & 28.8 \\
\hline \multirow[t]{2}{*}{$\begin{array}{l}\text { South pitted } \\
\text { plains }\end{array}$} & \multirow[t]{2}{*}{54} & \multirow[t]{2}{*}{7.50} & $\begin{array}{c}\text { Pure } \mathrm{CH}_{4} \\
\mathrm{~N}_{2}: \mathrm{CH}_{4} \\
\beta-\mathrm{N}_{2}\end{array}$ & A & $\begin{array}{c}3.4 \cdot 10^{-4} \\
0.018 \\
0.982\end{array}$ & $\begin{array}{c}0.492 \\
12.2 \\
1572.7 \\
\end{array}$ & 0.793 & $\begin{array}{c}0.03 \\
1.4 \\
77.9\end{array}$ \\
\hline & & & NSS endmember & $\mathrm{B}$ & 1.000 & 0.025 & 0.207 & 20.7 \\
\hline $\begin{array}{c}\text { West pitted } \\
\text { plains }\end{array}$ & 45 & 0.30 & $\begin{array}{c}\text { Pure } \mathrm{CH}_{4} \\
\mathrm{~N}_{2}: \mathrm{CH}_{4} \\
\end{array}$ & $\mathrm{~A}$ & $\begin{array}{l}0.009 \\
0.991 \\
\end{array}$ & $\begin{array}{l}1.949 \\
37.8 \\
\end{array}$ & 1.0 & $\begin{array}{c}0.9 \\
99.1 \\
\end{array}$ \\
\hline & & & DR & & & & & \\
\hline & & & $\mathrm{N}_{2}: \mathrm{CH}_{4}$ & $\mathrm{~A}$ & 1.0 & 1040.0 & 0.285 & 28.5 \\
\hline Baret Montes & 42 & 0.80 & $\begin{array}{l}\mathrm{NSS} \text { endmember } \\
\text { Crystalline } \mathrm{H}_{2} \mathrm{O}\end{array}$ & $\mathrm{B}$ & $\begin{array}{l}0.747 \\
0.252 \\
\end{array}$ & $\begin{array}{l}0.003 \\
0.019 \\
\end{array}$ & 0.715 & $\begin{array}{l}53.4 \\
18.1 \\
\end{array}$ \\
\hline Hillary & 45 & 060 & $\begin{array}{c}\text { Pure } \mathrm{CH}_{4} \\
\mathrm{~N}_{2}: \mathrm{CH}_{4}\end{array}$ & A & $\begin{array}{l}0.003 \\
0.997 \\
\end{array}$ & $\begin{array}{c}1.52 \\
296.6\end{array}$ & 0.389 & $\begin{array}{c}0.1 \\
38.7\end{array}$ \\
\hline Montes & 45 & 0.60 & $\begin{array}{l}\text { NSS endmember } \\
\text { Crystalline } \mathrm{H}_{2} \mathrm{O}\end{array}$ & $\mathrm{B}$ & $\begin{array}{l}0.630 \\
0.370\end{array}$ & $\begin{array}{l}0.005 \\
0.072\end{array}$ & 0.611 & $\begin{array}{l}38.5 \\
22.6\end{array}$ \\
\hline & & & Pure $\mathrm{CH}_{4}$ & A & 0.001 & 0.87 & 0.215 & $2.3 \cdot 10^{-2}$ \\
\hline $\begin{array}{l}\text { Al-Iaris1 } \\
\text { Montes }\end{array}$ & 42 & 0.50 & $\mathrm{~N}_{2}: \mathrm{CH}_{4}$ & A & 0.999 & 1277.3 & 0.215 & 21.5 \\
\hline & & & NSS endmember & $\mathrm{B}$ & 0.637 & 0.003 & 0.785 & $\mathbf{5 0 . 0}$ \\
\hline
\end{tabular}




\begin{tabular}{|c|c|c|c|c|c|c|c|c|}
\hline & & & Crystalline $\mathrm{H}_{2} \mathrm{O}$ & & 0.363 & 0.123 & 0.785 & 28.5 \\
\hline \multicolumn{9}{|c|}{ IBM } \\
\hline \multirow{3}{*}{ Baret Montes } & \multirow{3}{*}{52} & \multirow{3}{*}{2.80} & Pure $\mathrm{CH}_{4}$ & A & $\begin{array}{l}0.017 \\
0.983\end{array}$ & $\begin{array}{c}0.226 \\
33.9\end{array}$ & 0.317 & $\begin{array}{c}0.6 \\
31.1\end{array}$ \\
\hline & & & NSS endmember & & 0.478 & 0.007 & & 32.6 \\
\hline & & & Crystalline $\mathrm{H}_{2} \mathrm{O}$ & B & 0.522 & 0.17 & 0.683 & 35.7 \\
\hline \multirow{5}{*}{$\begin{array}{l}\text { Hillary } \\
\text { Montes }\end{array}$} & \multirow{5}{*}{52} & \multirow{5}{*}{6.00} & Pure $\mathrm{CH}_{4}$ & & $8.5 \cdot 10^{-6}$ & 0.271 & \multirow{3}{*}{0.455} & $3.9 \cdot 10^{-4}$ \\
\hline & & & $\mathrm{N}_{2}: \mathrm{CH}_{4}$ & A & $4.2 \cdot 10^{-4}$ & 24.2 & & 0.02 \\
\hline & & & $\beta-\mathrm{N}_{2}$ & & 1.000 & $375,246.0$ & & 45.5 \\
\hline & & & NSS endmember & & 0.339 & 0.007 & \multirow{2}{*}{0.545} & 18.5 \\
\hline & & & Crystalline $\mathrm{H}_{2} \mathrm{O}$ & B & 0.661 & 0.380 & & 36.0 \\
\hline \multirow{5}{*}{$\begin{array}{l}\text { Tenzing } \\
\text { Montes }\end{array}$} & \multirow{5}{*}{58} & \multirow{5}{*}{5.00} & Pure $\mathrm{CH}_{4}$ & & 0.001 & 0.521 & \multirow{3}{*}{0.606} & 0.03 \\
\hline & & & $\mathrm{N}_{2}: \mathrm{CH}_{4}$ & A & 0.029 & 20.2 & & 1.8 \\
\hline & & & $\beta-\mathrm{N}_{2}$ & & 0.970 & $1,513.5$ & & 58.8 \\
\hline & & & NSS endmember & B & 0.502 & 0.016 & 0394 & 19.8 \\
\hline & & & Crystalline $\mathrm{H}_{2} \mathrm{O}$ & B & 0.498 & 0.247 & 0.394 & 19.7 \\
\hline \multirow{4}{*}{$\begin{array}{c}\text { Al-Idrisi } \\
\text { Montes }\end{array}$} & \multirow{4}{*}{47} & \multirow{4}{*}{1.90} & $\mathrm{~N}_{2}: \mathrm{CH}_{4}$ & A & 0.057 & 11.97 & \multirow{2}{*}{0.437} & 2.5 \\
\hline & & & $\beta-N_{2}$ & A & 0.943 & 739.4 & & 41.2 \\
\hline & & & NSS endmember & B & 0.379 & 0.005 & 0563 & 21.3 \\
\hline & & & Crystalline $\mathrm{H}_{2} \mathrm{O}$ & B & 0.621 & 0.23 & 0.563 & 34.9 \\
\hline \multicolumn{9}{|c|}{ LPP } \\
\hline \multirow{5}{*}{$\begin{array}{l}\text { Hillary } \\
\text { Montes }\end{array}$} & & & Pure $\mathrm{CH}_{4}$ & & $2.1 \cdot 10^{-4}$ & 0.109 & & 0.01 \\
\hline & & & $\mathrm{N}_{2}: \mathrm{CH}_{4}$ & A & 0.036 & 7.312 & 0.592 & 2.1 \\
\hline & 53 & 9.10 & $\beta-\mathrm{N}_{2}$ & & 0.964 & 251.4 & & 57.1 \\
\hline & & & NSS endmember & B & 0.945 & 0.047 & & 38.5 \\
\hline & & & Crystalline $\mathrm{H}_{2} \mathrm{O}$ & B & 0.055 & 0.045 & 0.408 & 2.2 \\
\hline East pitted & 52 & 610 & $\mathrm{~N}_{2}: \mathrm{CH}_{4}$ & A & 0.014 & 4.95 & 10 & 1.4 \\
\hline plains & 52 & 0.10 & $\beta-\mathrm{N}_{2}$ & A & 0.986 & 761.6 & 1.0 & 98.6 \\
\hline South pitted & 44 & 04 & $\mathrm{~N}_{2}: \mathrm{CH}_{4}$ & A & 0.624 & 512.3 & 10 & 62.4 \\
\hline plains & 44 & 0.4 & $\beta-\mathrm{N}_{2}$ & A & 0.376 & 159.2 & 1.0 & 37.6 \\
\hline & & & $\mathrm{N}_{2}: \mathrm{CH}_{4}$ & A & 0.029 & 14.357 & 0790 & 2.3 \\
\hline $\begin{array}{l}\text { Cellular } \\
\text { nlains }\end{array}$ & 46 & 3.00 & $\beta-\mathrm{N}_{2}$ & A & 0.971 & 1727.4 & 0.190 & 76.7 \\
\hline & & & NSS endmember & $\mathrm{B}$ & 1.0 & 0.007 & 0.210 & 21.0 \\
\hline & & & Pure $\mathrm{CH}_{4}$ & & $3.0 \cdot 10^{-4}$ & 0.254 & & 0.02 \\
\hline & & & $\mathrm{N}_{2}: \mathrm{CH}_{4}$ & A & 0.031 & 6.33 & 0.516 & 1.6 \\
\hline $\begin{array}{l}\text { Al-Iaris1 } \\
\text { Montes }\end{array}$ & 49 & 4.90 & $\beta-\mathrm{N}_{2}$ & & 0.969 & 1217.2 & & 50.0 \\
\hline & & & NSS endmember & & 0.669 & 0.009 & & 32.3 \\
\hline & & & Crystalline $\mathrm{H}_{2} \mathrm{O}$ & B & 0.331 & 0.088 & 0.484 & 16.0 \\
\hline & & & $\mathbf{P I}$ & & & & & \\
\hline & & & Pure $\mathrm{CH}_{4}$ & & $2 . \cdot 10^{-4}$ & 0.738 & & 0.02 \\
\hline East pitted & 49 & 450 & $\mathrm{~N}_{2}: \mathrm{CH}_{4}$ & A & 0.023 & 13.6 & 0.730 & 1.7 \\
\hline plains & 49 & 4.50 & $\beta-N_{2}$ & & 0.977 & 2415.3 & & 71.3 \\
\hline & & & NSS endmember & $\mathrm{B}$ & 1.000 & 0.018 & 0.270 & 27.0 \\
\hline & & & Pure $\mathrm{CH}_{4}$ & & 0.001 & 0.346 & & 0.1 \\
\hline & & & $\mathrm{N}_{2}: \mathrm{CH}_{4}$ & A & 0.079 & 34.36 & 0.627 & 4.9 \\
\hline $\begin{array}{l}\text { Southwest } \\
\text { huffer }\end{array}$ & 57 & 5.20 & $\beta-N_{2}$ & & 0.920 & 834.1 & & 57.7 \\
\hline & & & NSS endmember & & 0.560 & 0.020 & & 20.9 \\
\hline & & & Crystalline $\mathrm{H}_{2} \mathrm{O}$ & B & 0.440 & 0.461 & 0.373 & 16.4 \\
\hline & & & $\mathrm{N}_{2}: \mathrm{CH}_{4}$ & A & 0.001 & 61.3 & 0668 & 0.1 \\
\hline plains & 48 & 2.10 & $\beta-N_{2}$ & A & 0.999 & $323,181.0$ & 0.008 & 66.7 \\
\hline & & & NSS endmember & B & 1.000 & 0.028 & 0.332 & 33.2 \\
\hline
\end{tabular}




\begin{tabular}{|c|c|c|c|c|c|c|c|c|}
\hline \multirow[t]{2}{*}{$\begin{array}{l}\text { Tenzing } \\
\text { Montes }\end{array}$} & \multirow[t]{2}{*}{58} & \multirow[t]{2}{*}{5.00} & $\begin{array}{c}\text { Pure } \mathrm{CH}_{4} \\
\mathrm{~N} 2: \mathrm{CH}_{4} \\
\beta-\mathrm{N}_{2}\end{array}$ & A & $\begin{array}{l}0.001 \\
0.014 \\
0.985\end{array}$ & $\begin{array}{c}0.416 \\
14.83 \\
2326.5\end{array}$ & 0.792 & $\begin{array}{c}0.04 \\
1.1 \\
78.1\end{array}$ \\
\hline & & & NSS endmember & B & 1.0 & 0.017 & 0.208 & 20.8 \\
\hline \multicolumn{9}{|c|}{ SH } \\
\hline \multirow{4}{*}{ Astrid Colles } & \multirow{4}{*}{48} & \multirow{4}{*}{4.80} & Pure $\mathrm{CH}_{4}$ & & $2.4 \cdot 10^{-5}$ & 23.65 & \multirow{3}{*}{0.676} & 0.002 \\
\hline & & & $\mathrm{N}_{2}: \mathrm{CH}_{4}$ & A & 0.020 & 10.26 & & 1.3 \\
\hline & & & $\beta-\mathrm{N}_{2}$ & & 0.980 & 1258.8 & & 66.3 \\
\hline & & & NSS endmember & B & 1.0 & 0.022 & 0.324 & 32.4 \\
\hline \multirow{4}{*}{$\begin{array}{c}\text { Challenger } \\
\text { Colles }\end{array}$} & \multirow{4}{*}{48} & \multirow{4}{*}{1.40} & Pure $\mathrm{CH}_{4}$ & & $1.87 \cdot 10^{-4}$ & 0.644 & \multirow{3}{*}{0.680} & 0.01 \\
\hline & & & $\mathrm{N}_{2}: \mathrm{CH}_{4}$ & A & 0.053 & 39.9 & & 3.6 \\
\hline & & & $\beta-\mathrm{N}_{2}$ & & 0.947 & 3397.6 & & 64.4 \\
\hline & & & NSS endmember & B & 1.0 & 0.006 & 0.320 & 32.0 \\
\hline \multirow{5}{*}{$\begin{array}{l}\text { Columbia } \\
\text { Colles }\end{array}$} & \multirow{5}{*}{52} & \multirow{5}{*}{4.60} & Pure $\mathrm{CH}_{4}$ & & $2.94 \cdot 10^{-5}$ & 0.337 & \multirow{3}{*}{0.456} & 0.001 \\
\hline & & & $\mathrm{N}_{2}: \mathrm{CH}_{4}$ & A & 0.001 & 11.849 & & 0.05 \\
\hline & & & $\beta-\mathrm{N}_{2}$ & & 0.999 & $124,708.0$ & & 45.5 \\
\hline & & & NSS endmember & B & 0.398 & 0.005 & 0544 & 21.7 \\
\hline & & & Crystalline $\mathrm{H}_{2} \mathrm{O}$ & B & 0.602 & 0.200 & 0.344 & 32.8 \\
\hline \multirow{4}{*}{ Soyuz Colles } & \multirow{4}{*}{47} & \multirow{4}{*}{4.30} & Pure $\mathrm{CH}_{4}$ & & $4.92 \cdot 10^{-5}$ & 1.044 & \multirow{3}{*}{0.803} & 0.004 \\
\hline & & & $\mathrm{N}_{2}: \mathrm{CH}_{4}$ & A & 0.016 & 13.221 & & 1.3 \\
\hline & & & $\beta-\mathrm{N}_{2}$ & & 0.984 & 2518.6 & & 79.0 \\
\hline & & & NSS endmember & B & 1.0 & 0.007 & 0.197 & 19.7 \\
\hline \multirow{4}{*}{$\begin{array}{l}\text { East pitted } \\
\text { plains }\end{array}$} & & & Pure $\mathrm{CH}_{4}$ & & $1.20 \cdot 10^{-4}$ & 0.448 & & 0.01 \\
\hline & 48 & 350 & $\mathrm{~N}_{2}: \mathrm{CH}_{4}$ & A & 0.026 & 16.927 & 0.796 & 2.0 \\
\hline & 48 & 3.50 & $\beta-\mathrm{N}_{2}$ & & 0.974 & 2041.8 & & 77.5 \\
\hline & & & NSS endmember & B & 1.0 & 0.013 & 0.204 & 20.4 \\
\hline & & & $\mathbf{S P}$ & & & & & \\
\hline & & & Pure $\mathrm{CH}_{4}$ & & $4.87 \cdot 10^{-4}$ & 0.319 & & 0.03 \\
\hline Hillary & 52 & 26 & $\mathrm{~N}_{2}: \mathrm{CH}_{4}$ & A & 0.070 & 23.20 & 0.700 & 4.9 \\
\hline Montes & 52 & 3.00 & $\beta-N_{2}$ & & 0.929 & 652.4 & & 65.1 \\
\hline & & & NSS endmember & $\mathrm{B}$ & 1.0 & 0.018 & 0.300 & $\mathbf{3 0 . 0}$ \\
\hline East pitted & 50 & 14 & $\mathrm{~N}_{2}: \mathrm{CH}_{4}$ & A & 0.127 & 70.4 & 1000 & 12.7 \\
\hline plains & 30 & 1.7 & $\beta-\mathrm{N}_{2}$ & $\mathrm{~A}$ & 0.873 & 198.6 & 1.000 & 87.3 \\
\hline South pitted & 44 & 050 & Pure $\mathrm{CH}_{4}$ & A & $2.9 \cdot 10^{-4}$ & 0.09 & 1000 & 0.03 \\
\hline plains & $4+$ & 0.00 & $\mathrm{~N}_{2}: \mathrm{CH}_{4}$ & $\pi$ & 0.990 & 322.1 & 1.000 & 99.0 \\
\hline North & & & Pure $\mathrm{CH}_{4}$ & & $2.5 \cdot 10^{-5}$ & 0.002 & & $2.5 \cdot 10^{-3}$ \\
\hline $\begin{array}{l}\text { pitted } \\
\text { plains }\end{array}$ & 48 & 0.80 & $\mathrm{~N}_{2}: \mathrm{CH}_{4}$ & A & 1.000 & 136.6 & 1.000 & 99.998 \\
\hline & & & Pure $\mathrm{CH}_{4}$ & & $2.67 \cdot 10^{-10}$ & 1.227 & & $1.9 \cdot 10^{-8}$ \\
\hline Cellular & 48 & 320 & $\mathrm{~N}_{2}: \mathrm{CH}_{4}$ & A & 0.038 & 11.86 & 0.731 & 2.8 \\
\hline plains & 40 & 0.20 & $\beta-\mathrm{N}_{2}$ & & 0.962 & 1124.0 & & 70.3 \\
\hline & & & NSS endmember & $\mathrm{B}$ & 1.0 & 0.011 & 0.269 & 26.9 \\
\hline & & & Pure $\mathrm{CH}_{4}$ & & $3.86 \cdot 10^{-4}$ & 0.409 & & 0.03 \\
\hline Southwest & 54 & 500 & N2:CH4 & A & 0.031 & 16.473 & 0.815 & 2.5 \\
\hline buffer & 54 & 5.00 & $\beta-\mathrm{N}_{2}$ & & 0.968 & 1010.4 & & 78.9 \\
\hline & & & NSS endmember & $\mathrm{B}$ & 1.0 & 0.026 & 0.185 & 18.5 \\
\hline West & & & Pure $\mathrm{CH}_{4}$ & & $4.0 \cdot 10^{-3}$ & 1.6 & & 0.4 \\
\hline $\begin{array}{l}\text { pitted } \\
\text { plains }\end{array}$ & 44 & 0.25 & $\mathrm{~N}_{2}: \mathrm{CH}_{4}$ & A & 0.996 & 57.9 & 1.000 & 99.6 \\
\hline
\end{tabular}

TBP

\begin{tabular}{|c|c|c|c|c|c|c|c|}
\hline Cellular & 45 & 1.30 & Pure $\mathrm{CH}_{4}$ & A & A $\quad 7.48 \cdot 10^{-10}$ & 0.252 & 0.616 \\
\hline
\end{tabular}




\begin{tabular}{ccccccc} 
plains & $\mathrm{N}_{2}: \mathrm{CH}_{4}$ & & 0.124 & 18.62 & & $\mathbf{7 . 6}$ \\
& $\beta-\mathrm{N}_{2}$ & & 0.876 & 2399.3 & & $\mathbf{5 3 . 9}$ \\
\hline \hline
\end{tabular}

\section{Spectroscopic Analysis}

365 The spectral indicators obtained from the methodology in Section 3, and the areal mass

366 fraction and grain size of the models' components, are presented as grayscale variation maps.

367 Dark grays are associated with suppressed band depths, or lower areal mass fraction and smaller 368 grain size, and light grays with stronger absorptions and higher areal mass fraction and grain size 369 values.

\section{1 $\mathrm{CH}_{4}$ and $\mathrm{N}_{2}$}

\subsection{1 $\mathrm{CH}_{4}$ band shifts}

$373 \mathrm{CH}_{4}$ and $\mathrm{N}_{2}$ are the two most abundant ices on Pluto' surface. Many studies (eg. Prokhvatilov

374 and Yantsevich, 1983; Lunine \& Stevenson, 1985; Protopapa et al., 2015) have showed that the

375 phase of the two ices, pure or diluted in each other, depends on the surface temperature. On

376 average, they are most likely to be found in two distinct phases on Pluto's surface (for an average

377 temperature of $44 \mathrm{~K}): \mathrm{CH}_{4}$ saturated in $\mathrm{N}_{2}$ ice $\left(\mathrm{CH}_{4}: \mathrm{N}_{2}\right)$ and $\mathrm{N}_{2}$ saturated in $\mathrm{CH}_{4}$ ice $\left(\mathrm{N}_{2}: \mathrm{CH}_{4}\right)$.

378 The $\mathrm{N}_{2}: \mathrm{CH}_{4}$ binary phase diagram, generated from X-ray diffraction experiments by

379 Prokhvatilov \& Yantsevich (1983), shows that at Pluto's surface temperature, the solubility limits

380 for the two phases are 3\% (of $\left.\mathrm{N}_{2}: \mathrm{CH}_{4}\right)$ and 5\% (of $\left.\mathrm{CH}_{4}: \mathrm{N}_{2}\right)$. The dilution of $\mathrm{CH}_{4}$ in the $\mathrm{N}_{2}$ matrix

381 produces a shift in the minima of the $\mathrm{CH}_{4}$ absorptions: the smaller the shift, the higher the $\mathrm{CH}_{4}$

382 abundance (Protopapa et al., 2015). 
To understand how, and in which ice phase, $\mathrm{CH}_{4}$ and $\mathrm{N}_{2}$ are distributed within the geologic

384 units of Sputnik Planitia, we calculated the shift in the LEISA spectra following the method

385 described in Section 3.4, and we show the result in Figure 6.

386 The highest values of the band shift index in the map refer to areas characterized by $\mathrm{N}_{2}$-rich

387 ice, while the lowest values indicate $\mathrm{CH}_{4}$-rich ice. The units with the highest index value are bct

388 in Al-Idrisi Montes and bcp in the Cellular plains. As a general trend, the geologic units in the

389 north and center of the mapping area, especially those within Al-Idrisi Montes and the Cellular

390 plains, have higher values of the shift index than units in the southwest: unit abm in Hillary

391 Montes and Zheng He Montes shows the lowest index values. The central cellular plains of

392 Sputnik Planitia (unit bcp) and unit bct in the northern part of Al-Idrisi Montes are the regions

393 richest in $\mathrm{N}_{2}$-rich ice. The amount of $\mathrm{N}_{2}$ slightly decreases from unit bcp to the northern areas of

394 the Cellular plains (units dcp and tbp), and to the lpp unit of the South and East pitted plains. The

395 shift index decreases - and consequently so does the $\mathrm{N}_{2}$-rich ice abundance - to the west,

396 incorporating units ibm and dmp in Hillary Montes, pmp and abm in Southwest buffer, and abm,

$397 \mathrm{ibm}$ and pmp in Tenzing Montes. The units covering the southernmost and easternmost areas of

398 the South and East pitted plains (dpp, pmp, and spp) have index values around 0.004, the

399 transition point between the $\mathrm{N}_{2}$-rich and $\mathrm{CH}_{4}$-rich ices. This north-to-south trend of increasing $\mathrm{N}_{2}$

400 abundance has previously been noted by S chmitt et al., (2017), and Protopapa et al. (2017).

$401 \quad$ Figure 6: Variation of the $\mathrm{CH}_{4}$ band shift index across the geologic units of Sputnik Planitia. The highest values of the band

402 shift index in the map (light grey) indicate areas characterized by $\mathrm{N}_{2}$-rich ice, while the lowest values (dark grey) indicate $\mathrm{CH}_{4}$ -

403 rich ice.

404

405 4.1.2 $\mathrm{CH}_{4}$ from LEISA spectra 
$\mathrm{CH}_{4}$ ice displays a large number of absorption bands in Pluto's spectra, organized in triplets or

407 in single broad bands (Figure 3 and Tables 2 and 3). We used equation 1 to calculate the

408 integrated band depths of each $\mathrm{CH}_{4}$ signature listed in Table 2, and we present them in Figure 7.

409 The areas with the most suppressed absorptions in almost all $\mathrm{CH}_{4}$ ranges considered - Range

410 1, Range 2, Range 4, and $1.48 \mu \mathrm{m}$ (Fig. 7 a,b,d,e) - are units drt and bct in Al-Idrisi Montes, and

411 units drt and abm in Baret Montes. Hillary and Tenzing Montes, on the other hand, show deeper

$412 \mathrm{CH}_{4}$ absorptions, with values similar to units bet and dcp. At $1.48 \mu \mathrm{m}$, units bep and dep of the

413 Cellular plains and unit lpp of the South and East pitted plains have similar band depth values. In

$414 \mathrm{CH}_{4}$ Range 1, Range 2, and Range 4 this similarity also extends to units lpp and pmp of the

415 South and East pitted plains.

416 In many aspects, the $\mathrm{CH}_{4}$ Range 3 map (Fig. 7c) appears to be the inverse of the other four

417 panels: the absorption bands are more suppressed in units bcp, dcp, and tbp of the Cellular

418 plains, where the intensities are similar to Al-Idrisi and Baret Montes, and in unit bct of Al-Idrisi

419 Montes. Furthermore, the $\mathrm{CH}_{4}$ absorption is deeper in the Southwest buffer and Tenzing Montes

420 provinces.

421 Schmitt et al. (2017) found the same trend observed here for the four ranges of $\mathrm{CH}_{4}$ triplets:

422 the intensity of the $\mathrm{CH}_{4}$ bands is high inside units bcp and dep of the Cellular plains in Ranges 1,

4232 , and 4, whereas the strength of the band in Range 3 decreases in the very same areas, but not in

424 the southwestern territories corresponding to the Southwest buffer and Tenzing Montes

425 provinces. 
Figure 8 displays the amount of $\mathrm{CH}_{4}$ diluted in the $\mathrm{N}_{2}$-rich phase (panel a) and the

433 temperatures (panel b) estimated by the models. The maps in Figure $8 \mathrm{a}$ and $8 \mathrm{~b}$ show that the

434 amount of $\mathrm{CH}_{4}$ diluted in $\mathrm{N}_{2}$ and the temperature are both very low in the northern, western, and

435 central regions of Sputnik Planitia, and increase in the south and west. A similar trend was

436 already found by Protopapa et al. 2017 (see their figure 5 panel C). The highest temperature and

$437 \mathrm{CH}_{4}$ content are found in unit lpp of Hillary Montes. Here the concentration of $\mathrm{CH}_{4}$ reaches

$4389.1 \%$, with an estimated temperature of $53 \mathrm{~K}$. Units dpp in South pitted plains, lpp in East pitted

439 plains, ibm in Hillary Montes, pmp and spp in Southwest buffer, and pmp and ibm in Tenzing

440 Montes have $\mathrm{CH}_{4}$ content between 5\% and 7\%, and high temperatures, between 52 and $58 \mathrm{~K}$.

441 The remaining provinces across the mapping area show $\mathrm{CH}_{4}$ content below $5 \%$, reaching a

442 minimum in unit lpp of South pitted plains $(0.4 \%)$, dpp of West pitted plains $(0.3 \%)$ and abm of

443 Tenzing Montes (0.05\%). $\mathrm{CH}_{4}$ in unit dep in the Cellular plains accounts for $0.06 \%$, which

444 increases to $1.3 \%$ and $1.7 \%$ in units tbp and dcp respectively.

445 Units abm in Zheng He Montes and pmp in Tenzing Montes, Baret Montes, and Southwest

446 buffer have the highest temperatures, ranging between $55 \mathrm{~K}$ and $58 \mathrm{~K}$. Units drt, bct and dcp, on

447 the other hand, show temperatures ranging between 43 and $45 \mathrm{~K}$.

448 The fraction of $\mathrm{CH}_{4}$ diluted in $\mathrm{N}_{2}$ estimated in the models, and the corresponding predicted

449 temperatures are consistent with the results from Protopapa et al. (2017), which showed a 450 dilution content of $\mathrm{CH}_{4}$ in $\mathrm{N}_{2}$ in the Sputnik Planitia region ranging between $0.1 \%$ and $5 \%$.

451 Pure $\mathrm{CH}_{4}$ can also be found in Sputnik Planitia but always in very small amounts, below $1.5 \%$

452 (Figure 9a and b). The grain size ranges between 0.004 and $2 \mathrm{~mm}$. Only one unit shows a larger

453 grain size, sh in Astrid Colles (23.6 mm). 


\subsection{4 $\mathrm{N}_{2}$ from LEISA spectra}

$\mathrm{N}_{2}$ was discovered on the surface of Pluto through the detection of its absorption band at 2.15 $\mu \mathrm{m}$ (Owen et al., 1993), located in a spectral region dominated by the $\mathrm{CH}_{4}$ Range 4 absorption

464 (Figure 3). This band is relatively weak, and the fact that it is clearly observed in almost all 465 Pluto's spectra means that the $\mathrm{N}_{2}$ layer is thick enough to allow the light to travel for a significant 466 path length (on the order of centimeters) before being absorbed or reflected (Grundy et al., 1993;

467 Cruikshank et al., 2015). The strong absorptions of $\mathrm{CH}_{4}$, on the other hand, only need a layer 468 some microns thick to be clearly observable in a spectrum. $\mathrm{N}_{2}$ is, therefore, the most abundant 469 ice on Pluto's surface, and it is contaminated by smaller amounts of $\mathrm{CH}_{4}, \mathrm{CO}$ and other minor 470 constituents (Cruikshank et al., 1984).

471 The $2.15 \mu \mathrm{m}$ integrated band depth variation map is shown in Figure 10.

472 The deepest $\mathrm{N}_{2}$ absorptions are localized in small areas, namely in unit bct at the northern end 473 of Al-Idrisi Montes, and in spp of the West pitted plains. Unit bcp of the Cellular plains, and 474 units lpp and spp in the South pitted plains, also show deep $\mathrm{N}_{2}$ absorptions. The band intensity 475 decreases northwards, in units dcp and tbp of the Cellular plains, eastward, in units lpp, dpp and 476 pmp of the E pitted plains, and southwards, in units dpp and pmp of the S pitted plains. The 477 western areas have, on average, weaker or even null $\mathrm{N}_{2}$ absorption. In fact, unit abm in Hillary 478 and Tenzing Montes shows negligible amounts of $\mathrm{N}_{2}$. Low $\mathrm{N}_{2}$ content is also observed in units 479 abm and drt of Al-Idrisi Montes, abm of Zheng He Montes, and all the units of Baret Montes, 480 Southwest buffer and Tenzing Montes. In the east, Challenger Montes show similar, suppressed 481 intensities, standing out from its surroundings. A comparison with Figure 6, shows that the very 
482 same provinces with weak $\mathrm{N}_{2}$ absorptions also have small values of the shift parameter,

483 confirming the prevalence of $\mathrm{CH}_{4}$-rich ice.

\subsection{5 $\mathrm{N}_{2}$ from model spectra}

488 For the temperature values estimated by the models, the phase diagram suggests that most $\mathrm{N}_{2}$ 489 within Sputnik Planitia is in the form of $\mathrm{N}_{2}: \mathrm{CH}_{4}$ ice. The $\mathrm{N}_{2}$-rich phase can coexist with 490 increasing amounts of pure $\mathrm{N}_{2}$ in the $\beta$-form if the temperature rises.

491 Figure 11 and Figure 12 show the areal mass fraction, in the "A" panels, and the grain size, in 492 the "B" panels, of $\mathrm{N}_{2}: \mathrm{CH}_{4}$ ice and of pure $\beta-\mathrm{N}_{2}$ respectively.

493 From Figure 11a, we can observe high amounts of $\mathrm{N}_{2}: \mathrm{CH}_{4}$ covering the very central regions

494 of Sputnik Planitia, corresponding to units bcp in the Cellular plains and lpp and spp of the South 495 pitted plains. In the West, units abm of Al-Idrisi Montes, dpp of West pitted plains and Hillary 496 Montes, abm of Southwest buffer and Tenzing Montes also show high abundance of $\mathrm{N}_{2}$. In the 497 remaining territories, the areal mass fraction of $\mathrm{N}_{2}$-rich ice decreases sharply, dropping below 498 10\%. A similar trend was observed in Protopapa et al. (2017). However, the difference in the 499 absolute numbers of abundance are to be attributed to differences in the modeling (e.g., Hapke 500 parameters, areal versus intimate mixture). The geologic units where $\mathrm{N}_{2}: \mathrm{CH}_{4}$ abundance is low, 501 though, correspond to areas where pure $\beta-\mathrm{N}_{2}$ has high concentrations (Figure 12a). Units dcp and 502 tbp in the northern part of the Cellular plains, bct and ibm in Al-Idrisi Montes, abm, ibm and spp 503 in Hillary Montes, pmp and and spp in Southwest buffer, ibm and pmp in Tenzing Montes, and 504 lpp, dpp, spp, and pmp of East and South pitted plains have pure $\beta-\mathrm{N}_{2}$ areal mass fraction 505 ranging between $35.4 \%$ and $98.6 \%$. 
The grain size of the $\mathrm{N}_{2}: \mathrm{CH}_{4}$ ice is below $200 \mathrm{~mm}$ in most provinces. Some units in the west

507 show, on the other hand, coarser grain sizes, reaching $800 \mathrm{~mm}$. For pure $\beta-\mathrm{N}_{2}$, the grain size is

508 always very large, on average above $350 \mathrm{~mm}$.

\subsubsection{Distribution of $\mathrm{CH}_{4}$-rich and $\mathrm{N}_{2}$-rich ices}

514 The distribution of $\mathrm{CH}_{4}$-rich and $\mathrm{N}_{2}$-rich ices can be inferred by integrating the abundance

515 maps shown previously with correlation plots between the $\mathrm{CH}_{4}$-band shift index and the $\mathrm{CH}_{4}$ and

$516 \quad \mathrm{~N}_{2}$ integrated band depths.

517 Figure 13a shows the scatter plots of the $\mathrm{CH}_{4}$ integrated band depths in Ranges 1 (top panel),

5182 (middle panel), and 4 (bottom panel) versus the $\mathrm{CH}_{4}$ shift index. We grouped these spectral

519 indices together because they show similar trends with the band position index. The points are 520 organized in two groups, separated in the plots by a dotted line. The first group (G1), above the

521 line, shows increasing band strength with increasing values of the shift parameter. The second 522 group (G2), below the dotted line, also shows a positive trend, but the points are more dispersed.

523 Furthermore, the values of the $\mathrm{CH}_{4}$ integrated band depths in $\mathrm{G} 2$ are lower than the units in $\mathrm{G} 1$.

524 The Pearson correlation coefficient calculated for the data belonging to G1 and G2 confirm the 525 positive correlations. For $\mathrm{G} 1, \mathrm{CH}_{4}$ Range 1 has a moderate correlation with the $\mathrm{CH}_{4}$ shift $(\rho=$ $5260.44)$, while for Range $2(\rho=0.78)$ and Range $4(\rho=0.82)$ the correlation coefficient has higher

527 values. The points belonging to G2 appear more dispersed, but a positive trend is still visible.

528 The correlation coefficient is $\rho=0.24$ for the Range $1, \rho=0.62$ for the Range 2 , and $\rho=0.74$ for 529 the Range 4. 
The units included in G1 show increasing shift index with integrated band depths associated

531 with a west-east trend. The units covering the western provinces show the most suppressed $\mathrm{CH}_{4}$

532 absorptions in Ranges 1, 2, and 4, and smaller values of the shift index. Moving toward the

533 central and eastern territories, the two spectral parameters increase in value.

534 Group G2 includes the following units, in order of increasing values of the shift index and

535 integrated band depths: drt, ibm, abm of Baret Montes, drt of Al-Idrisi Montes, sh of Columbia

536 Colles, drt of Hillary Montes, abm of Al-Idrisi Montes, tbp of the Cellular plains, and lpp, ibm,

537 bct, and dcp of Al-Idrisi Montes. All these units, excepting sh and tbp, are located on the western

538 edge of Sputnik Planitia.

539 The depth of an absorption band is controlled by several physical parameter, primarily the

540 amount of ice, grain diameter, and the single particle phase function. Generally, the greater the

541 amount of ice, or the larger the grain size, or the more a grain scatters incident sunlight

542 preferentially in the forward direction (Verbiscer et al., 2006), the deeper the absorptions.

543 To examine the influence of the scattering on the best fit models, we examined the H-band

$544 \quad(1.59-1.82 \mu \mathrm{m})$ range of the spectrum from spp unit of North Pitted Plains. We used this

545 spectrum because the surface is mostly smooth (i.e. no mountains slopes) and likely minimizes

546 changes in the spectrum due to phase, emission or incidence angles. We find that changes in

547 the Henyey-Greenstein $g$-value affects best-fit parameters like temperature and dilution.

548 Because we adopt the global value for the Henyey-Greenstein $g$-value from Verbiscer et al

549 (2019) which is based on a fit to visible, not near-infrared, data at $0.55 \mu \mathrm{m}$, our best-fit values for

550 temperature and dilution should be considered preliminary, as additional analysis would be

551 required to understand the local $g$-values in the H-band in each region we examined. For the 
552 scope of this paper, we will focus on abundance and grain size variation as the main parameters

553 controlling the spectra shape and the bands depth.

554 Based on the abundance relationship alone, we might be led to conclude from Figure 13a that

555 the increase in $\mathrm{CH}_{4}$ band strength with the $\mathrm{CH}_{4}$-shift index is due to an increase in $\mathrm{CH}_{4}$

556 abundance. When the shift index was presented in section 3.2, though, we specified that higher

557 index values correspond to lower amounts of $\mathrm{CH}_{4}$-rich ice. Therefore, a trend in abundance,

558 opposite of what observed in Figure 13a, is expected if grain scattering properties are not taken

559 into account: the $\mathrm{CH}_{4}$ integrated band depth should be less intense - or, the abundance of $\mathrm{CH}_{4}$

560 should decrease - with increasing values of the shift index. This trend, then, can be explained by

561 a difference in the scattering properties of particles within spectral Ranges 1, 2, and 4.

562 Figure 13b displays the integrated band depths of $\mathrm{CH}_{4}$ Range 3 (top panel), $1.48 \mu \mathrm{m}$ band

563 (middle panel), and $\mathrm{N}_{2}$ absorption band at $2.15 \mu \mathrm{m}$ (bottom panel). In contrast to the three plots

564 in Figure 13a, these spectral parameters show a clear and single trend with the $\mathrm{CH}_{4}$ shift index.

565 As we observed in Figure 7, the $\mathrm{CH}_{4}$ Range 3 absorption shows a very different abundance

566 distribution across Sputnik Planitia compared to the other $\mathrm{CH}_{4}$ absorptions. This discrepancy is

567 also reflected in the plots showed in Figure 13b, where the intensity of the band shows a decrease

568 in strength with increasing band shift, consistent with the definition of the $\mathrm{CH}_{4}$ shift index. The

569 Pearson correlation coefficient between Range 3 and the shift index is equal to -0.67 , proving

570 that an anti-correlation exists. We also observe that the band intensity decreases from the western

571 to the central and eastern units, indicating a higher abundance of $\mathrm{CH}_{4}$-rich ice in the western

572 mountain ranges. Some exceptions are observed: units abm, ibm, and lpp of Al-Idrisi Montes

573 have $\mathrm{CH}_{4}$ absorption values in Range 3 similar to the Cellular plains, South and East pitted

574 plains, and the four Colles provinces, while units bct and dcp have the most suppressed band 
575 values. The scatter plot of the $\mathrm{CH}_{4}$ band at $1.48 \mu \mathrm{m}$ as a function of the $\mathrm{CH}_{4}$ band shift

576 results in an elongated cloud, without any measurable slope, but rather a flat trend. The

577 correlation coefficient is indeed $\rho=-0.12$. Finally, the bottom panel of Figure $13 b$ shows a

578 positive correlation $(\rho=0.68)$ between the intensity of the $\mathrm{N}_{2}$ band at $2.15 \mu \mathrm{m}$ and the $\mathrm{CH}_{4}$ band

579 shift: the abundance of $\mathrm{N}_{2}$-rich ice increases where the $\mathrm{CH}_{4}$-shift index has higher values.

580 The unexpected behavior of the strongest $\mathrm{CH}_{4}$ absorptions (Ranges 1, 2, 4) has been

581 previously observed, mainly in Schmitt et al. (2017). The authors compared the strength of the

$582 \mathrm{CH}_{4}$ main absorptions with a $\mathrm{CH}_{4}$-band shift parameter and observed that the data relative to

583 Sputnik Planitia were offset with respect to the rest of Pluto's surface.

584 The absorptions plotted in Figure 13A do not increase, or decrease, in depth linearly with a

585 variation of abundance of $\mathrm{CH}_{4}$, grain size or forward scattering, but they anyway tend to saturate

586 quickly with increasing abundance of $\mathrm{CH}_{4}$, or with increasing grain size or forward scattering.

587 Therefore, these three ranges cannot reliably be used to infer the $\mathrm{CH}_{4}$ abundance and, in turn, to

588 understand if abundance or grain size can in fact explain the unexpected trend. The absorption in

589 Range 3 and the band centered at $1.48 \mu \mathrm{m}$ are weaker, and a larger quantity of $\mathrm{CH}_{4}$ would be

590 necessary to make them saturated. Therefore, the plots in Figure 13B are more reliable for

591 understanding how $\mathrm{CH}_{4}$-rich and $\mathrm{N}_{2}$-rich ices distribute across Sputnik Planitia. Furthermore, the

592 trends in Figure 13B are consistent with the results from the models shown in Figures 8, 11 and

593 12. The geologic units with the lowest value of the shift index are mostly located in the

594 southwestern mountain ranges, mainly Hillary and Zheng He Montes. Indeed, the models for

595 units abm and ibm of these areas show the highest concentration of $\mathrm{CH}_{4}$-rich ice. The central

596 part of the Cellular plains and the East and South pitted plains show high abundance of $\mathrm{N}_{2}: \mathrm{CH}_{4}$

597 ice or of pure $\beta-\mathrm{N}_{2}$, as well as high values of $\mathrm{CH}_{4}$-shift index and of $\mathrm{N}_{2}$ integrated band depth. 
The hypothesis we can formulate to understand the trends of Figure 13A, based on the data shown thus far, is that $\mathrm{N}_{2}$ and $\mathrm{CH}_{4}$ are mixed with, or in close contact with patches of, some

600 contaminants, or that there are differences in the single particle phase functions. The effect of 601 these materials or surface particles that are more backscattering would be to suppress the $\mathrm{CH}_{4}$ 602 band depths - leaving the band shift unaltered - on the western regions where they may be more 603 exposed and, therefore, more abundant. The $\mathrm{CH}_{4}$ band at $1.48 \mu \mathrm{m}$ is minimally affected by 604 contaminants since its trend represents a transition between the absorptions in Figure 13A and 605 the $\mathrm{CH}_{4}$ absorption in Range 3 of Figure 13b. This last feature doesn't seem to be sensitive to 606 contaminants, but rather to a variation in the abundance of $\mathrm{CH}_{4} \mathrm{Or} \mathrm{N}_{2}$ ices.

607 To support this idea, we plotted the $\mathrm{CH}_{4}$ integrated band depths in Ranges 1, 2, and 4 against 608 the $\mathrm{CH}_{4}$ integrated band depth in Range 3 (Figure 14). The three plots clearly show a lack of 609 correlation between the represented parameters, the Pearson coefficients being $\rho=0.34$ (Range 610 1), $\rho=0.11$ (Range 2), and $\rho=0.01$ (Range 4). The low or null correlations suggest that the 611 physical property controlling the depth of the $\mathrm{CH}_{4}$ absorptions in Ranges 1,2 , and 4 is not 612 affecting the absorption in Range 3. Because of the different sensitivity of the four ranges to the 613 variation in $\mathrm{CH}_{4}$ ice abundance and grain size, we can exclude these two physical parameters as 614 the main reason for the trend observed in Figure 13a.

615 It is worth noticing from Figure 14 that units bet and dep in Al-Idrisi Montes have the most 616 suppressed absorptions in Range 3, but quite intense values in Ranges 1, 2, and 4. Indeed, these 617 two units show the deepest bands among the units belonging to group G2 (Figure 13A). Unit drt 618 in Baret and Al-Idrisi Montes has very suppressed bands in all $\mathrm{CH}_{4}$ ranges, while in Hillary 619 Montes the same unit has quite deep absorption in Range 3, but weak intensities in the other $\mathrm{CH}_{4}$ 620 ranges. 
Fig 13: (A) Scatter plot showing the correlation between the $\mathrm{CH}_{4}$ integrated band depth in Ranges 1 (top), 2 (middle), and (bottom) with the $\mathrm{CH}_{4}$ shift index. The data are split into two groups, separated by a dotted curve. The points belonging to group G1, above the curve, show increasing band strength with increasing values of the shift parameter. The second group (G2), below the dotted line, also shows a positive trend, but the points are more dispersed. Furthermore, the values of the $\mathrm{CH}_{4}$ integrated band depths in $G 2$ are lower than the units in G1. The positive trends in both groups contradicts the definition of the $\mathrm{CH}_{4}$ shift index, i.e. higher index values correspond to lower of $\mathrm{CH}_{4}$-rich ice amount. Therefore, a trend opposite of what observed in panel $A$ is expected: the $\mathrm{CH}_{4}$ integrated band depth should be less intense - or, the abundance of methane should decrease - with increasing values of the shift index. (B) Scatter plot showing the correlation between the $\mathrm{CH}_{4}$ integrated band depth in Range 3 (top), at $1.48 \mu \mathrm{m}$ (middle), and the N2 integrated band depth at $2.15 \mu \mathrm{m}$ (bottom) with the $\mathrm{CH}_{4}$ shift index. As opposite to the three plots in panel A, these spectral parameters show a clear and single trend with the $\mathrm{CH}_{4}$ shift index, in agreement with the definition of the $\mathrm{CH}_{4}$ shift index.

Fig 14: Scatter plot showing the correlation between the $\mathrm{CH}_{4}$ integrated band depths in Ranges 1 (top), 2 (middle), and 4 (bottom) versus the $\mathrm{CH}_{4}$ integrated band depth in Range 3. The plots clearly show a lack of correlation between the represented parameters, indicating that the factor influencing the depth of the $\mathrm{CH}_{4}$ absorptions in Ranges 1, 2, and 4 doesn't have any (or it has negligible) effect on the absorption in Range 3.

\section{$4.2 \mathrm{CO}$}

Figure 15 displays the spatial distribution of the CO absorption across Sputnik Planitia, centered at $1.58 \mu \mathrm{m}$. The $\mathbf{C O}$ absorption is generally weak; the deepest ones occur in units abm in Southwest buffer and units lpp and spp on the eastern perimeter of the Cellular plains. Unit bcp in the Cellular plains, units ibm and pmp in Tenzing Montes, and units lpp and spp in the South and East pitted plains, also show high $\mathrm{CO}$ content. The band intensity decreases in the northern portion of Sputnik Planitia, in units dcp and tbp, and in the eastern and southern units of the South and East pitted plains, dpp and pmp. The regions mostly depleted in CO (where the integrated band depth is close to 0) are units abm in Zheng He and Hillary Montes, and drt in Baret Montes. CO content in Al-Idrisi Montes is low and quite constant in all its geologic units, excepting bct, where the band intensity decreases even more. The $\mathrm{CO}$ distribution in the Cellular plains, South and East pitted plains, Hillary Montes, and Baret Montes closely resembles the $\mathrm{N}_{2}$ trend observed in Figure 11. Other areas, on the other hand, show an anti-correlation with the $\mathrm{N}_{2}$ distribution. The most remarkable differences are observed for units bct and ibm in Al-Idrisi Montes, in the North and West pitted plains, and in the abm units of the Cellular plains, Southwest buffer and Tenzing Montes. 
654 More complex is the comparison with the $\mathrm{CH}_{4}$ integrated band depth maps of Figure 7. As we

655 described before, $\mathrm{CH}_{4}$ absorptions are deeper in Ranges 1, 2, 4 and in the $1.48 \mu \mathrm{m}$ band in the

656 Cellular plains and South and East pitted plains provinces, and in some units of Hillary Montes

657 and Southwest buffer. Units abm and drt of Al-Idrisi and Baret Montes instead show the weakest

$658 \mathrm{CH}_{4}$ absorptions. The $\mathrm{CO}$ integrated band depth map shows the same trend in the very same

659 provinces. $\mathrm{CH}_{4}$ Range 3, on the other hand, shows the opposite results, the band being more

660 intense where the other $\mathrm{CH}_{4}$ and the $\mathrm{CO}$ absorptions are shallower.

661 We investigated the correlation between $\mathrm{CH}_{4}$ and $\mathrm{N}_{2}$ absorptions with $\mathrm{CO}$ in Figure 16. The

662 scatter plot of $\mathrm{CH}_{4}$ in Ranges 1, 2, and 4 versus the $\mathrm{CO}$ integrated band depth is displayed in

663 Figure 16a. On average, $\mathrm{CO}$ correlates well to the three $\mathrm{CH}_{4}$ absorptions, the Pearson linear

664 correlation coefficient being 0.64 for Range 1, 2, and 4. We notice, though, that the units do not

665 distribute in the same way, but they can be split into two groups, separated in the three panels of

666 Figure 16a by a vertical dotted line. The units on the left side have a sparse distribution and no

667 apparent trend. They are all located on the western edge of Sputnik Planitia, excepting unit tbp in

668 the Cellular plains and unit sh in Columbia Colles. The points on the right portion of the plots

669 belong to units in the central and eastern parts of Sputnik Planitia, excepting the Southwest

670 buffer and Tenzing Montes. For these units, the correlation between the integrated band depths

671 of $\mathrm{CO}$ and $\mathrm{CH}_{4}$ is also not evident, having a flat distribution.

672 Figure $16 \mathrm{~b}$ shows the $\mathrm{CO}$ integrated band depth plotted against the integrated band depth of

$673 \mathrm{CH}_{4}$ in Range 3 and at $1.48 \mu \mathrm{m}$, and of $\mathrm{N}_{2}$. The only clear correlation occurs between $\mathrm{CO}$ and

674 the band at $1.48 \mu \mathrm{m}$, with $\rho=0.53$, while the correlation coefficient for $\mathrm{CH}_{4}$ band in Range 3 and

$675 \mathrm{~N}_{2}$ are negligible, being 0.09 and 0.12 respectively. 
If we compare these results with Figure 13, we can notice some similarities. $\mathrm{CH}_{4}$ in Ranges 1,

677 2, and 4 showed an unexpected trend in Figure 13a, the absorptions becoming deeper with

678 increasing values of the $\mathrm{CH}_{4}$ shift parameter. The abundance of $\mathrm{CH}_{4}$ or its grain size cannot

679 explain the discrepancy, since they weakly influence the depth of those bands. $\mathrm{CH}_{4}$ in Range 3

680 and $\mathrm{N}_{2}$, on the other hand, have a trend consistent with the definition of the $\mathrm{CH}_{4}$ shift index: low

681 values of the index indicate dominance of $\mathrm{CH}_{4}$-rich ice, while high values of the index imply

682 high abundances of $\mathrm{N}_{2}$-rich ice.

683 Comparing Figure 13a with Figure 16a, we observe that geologic units showing deeper $\mathrm{CH}_{4}$

684 absorptions and higher values of the $\mathrm{CH}_{4}$ shift index also have strong $\mathrm{CO}$ bands. Similarly, units

685 having a random distribution in Figure 13a (group G2) also have a sparse distribution and low

$686 \mathrm{CO}$ values in Figure 16a (on the left side of the dotted line). The top and bottom panels of

687 Figure 16b are not useful for any definitive interpretation, since the $\mathrm{CO}$ integrated band

688 depth doesn't have any clear correlation with either integrated band depth of $\mathrm{CH}_{4}$ in Range 6893 or $\mathbf{N}_{2}$.

690 Grundy et al. (2013, 2014), and Schmitt et al. (2017) noticed that CO is almost absent in areas

691 dominated by $\mathrm{CH}_{4}$-rich ice, but it is abundant in $\mathrm{N}_{2}$-rich areas. The comparison of the $\mathrm{CH}_{4}$ shift

692 index map in Figure 6 with the CO map in Figure 15 leads to the confirm of the authors'

693 conclusion for almost all geologic units in Sputnik Planitia, with a few exceptions. Units bet in

694 Al-Idrisi Montes, drt in Baret and Hillary Montes, and dpp and spp in the West pitted plains are

695 the most evident: $\mathrm{CO}$ absorption is suppressed where the shift indicates dominance of $\mathrm{N}_{2}$-rich

696 ice.

697 The correlation between $\mathrm{CO}$ and $\mathrm{N}_{2}$ is also evident when comparing Figure 15 with the

$698 \mathrm{~N}_{2}: \mathrm{CH}_{4}$ (Figure 11a) and pure $\beta-\mathrm{N}_{2}$ (Figure 12a) maps. Units with high amounts of $\mathrm{N}_{2}$-rich ices, 
699 like bcp and dcp in the Cellular plains, and spp in South pitted plains, or with considerable

700 presence of pure $\beta-\mathrm{N}_{2}$, such as lpp in East pitted plains, have strong CO intensities. We also

701 notice that the $\mathrm{CO}$ band is weaker where no pure $\beta-\mathrm{N}_{2}$ is observed, like Zheng He Montes, and

702 drt in Baret Montes.

$703 \quad$ Fig. 15: Map of variation of CO integrated band depth at $1.58 \mu \mathrm{m}$.

704

705

706

707

708

709

710

711

712

Fig 16: (A) Scatter plot showing the correlation between the $\mathrm{CH}_{4}$ integrated band depth in Ranges 1 (top), 2 (middle), and 4

(bottom) with the CO integrated band depth. Because rhe data shows to different trends, we split them into two groups, separated

by a vertical dotted line. The units on the left side have a positive trend but a sparse distribution, the points on the right portion

of the plots show a flat distribution. (B) Scatter plot showing the correlation between the $\mathrm{CH}_{4}$ integrated band depth in Range 3

(top), at $1.48 \mu \mathrm{m}$ (middle), and the $\mathrm{N}_{2}$ integrated band depth at $2.15 \mu \mathrm{m}$ (bottom) with the CO integrated band depth. The only

clear correlation occurs between $\mathrm{CO}$ and the band at $1.48 \mu \mathrm{m}$, while the correlation coefficient for $\mathrm{CH}_{4}$ band in Range 3 and $\mathrm{N}_{2}$

are negligible.

713 The presence of crystalline $\mathrm{H}_{2} \mathrm{O}$ ice on Pluto's surface was investigated and proved by

714 numerous works, e.g. Grundy et al. (2016), Protopapa et al. (2017); Cook et al. (2019), and

715 Cruikshank et al. (2020).

716 In our models we consider $\mathrm{H}_{2} \mathrm{O}$ ice in two forms, crystalline and amorphous.

717 The spatial variation of the crystalline $\mathrm{H}_{2} \mathrm{O}$ ice as predicted in the models is presented in

718 Figure 17. The left panel (Figure 17a) depicts the areal mass fraction, the right panel (Figure

719 17b) the grain size. The mountain ranges extending along the western boundary of Sputnik

720 Planitia are the only provinces hosting crystalline $\mathrm{H}_{2} \mathrm{O}$ ice, excepting Columbia Colles in the

721 east. The $\mathrm{H}_{2} \mathrm{O}$ ice abundance ranges between $2 \%$ and $42 \%$.

722 Unit abm in the mountain ranges of Baret and Hillary Montes show the highest percentage of

723 crystalline $\mathrm{H}_{2} \mathrm{O}$ ice (37\% to $42 \%$ ). Al-Idrisi Montes displays a lower percentage, ranging

724 between $\sim 25 \%$ and $\sim 35 \%$ across its constituent units. The units in Southwest buffer and Tenzing

725 Montes, and the drt unit in Baret Montes, show a decrease in crystalline $\mathrm{H}_{2} \mathrm{O}$ ice, being between

$726 \sim 9 \%$ and $\sim 18 \%$. The grain size in all the units is smaller than $6 \mathrm{~mm}$. The largest grains are found 
727 in the Southwest buffer, followed by Hillary and Tenzing Montes. In all the other units, the

728 crystalline $\mathrm{H}_{2} \mathrm{O}$ ice grain size is estimated to be smaller than $0.2 \mathrm{~mm}$.

729 The estimate of the amount of $\mathrm{H}_{2} \mathrm{O}$ ice from LEISA data is not a trivial task. Indeed, the main

$730 \mathrm{H}_{2} \mathrm{O}$ ice absorptions occur at $1.5 \mu \mathrm{m}$, the location of the gap in the $\mathrm{CH}_{4}: \mathrm{N}_{2}$ optical constants, and

731 at $2.02 \mu \mathrm{m}$, where $\mathrm{CH}_{4}$ shows stronger absorptions with increasing wavelengths. Furthermore,

732 the absorption around $2.0 \mu \mathrm{m}$ is partly cut out by the optical constants gap. Keeping this in mind,

733 we calculated the $\mathrm{H}_{2} \mathrm{O}$ integrated band depth by applying equation 1 with the parameters listed in

734 Table 2 for the spectra of the geologic units having crystalline $\mathrm{H}_{2} \mathrm{O}$ predicted by the models.

735 Fig. 17: Maps of variation of Crystalline $\mathrm{H}_{2} \mathrm{O}$ areal mass fraction (A) and grain size (B) estimated by the spectral modeling.

737 Figure 18 shows the mutual variation of the crystalline $\mathrm{H}_{2} \mathrm{O}$ areal mass fraction, the $\mathrm{H}_{2} \mathrm{O}$

738 integrated band depth, and the $\mathrm{CH}_{4}$ shift parameter. Crystalline $\mathrm{H}_{2} \mathrm{O}$ ice correlates well to the

$739 \mathrm{H}_{2} \mathrm{O}$ integrated band depth (top, left panel; $\rho=0.55$ ). No correlation, on the other hand, exists

740 between the $\mathrm{H}_{2} \mathrm{O}$ areal mass fraction (top, right panel), the $\mathrm{H}_{2} \mathrm{O}$ integrated band depth (bottom,

741 left panel), and the shift parameter $(\rho=-0.04$ and $\rho=-0.05)$, suggesting that $\mathrm{H}_{2} \mathrm{O}$ does not

742 influence the $\mathrm{CH}_{4}$ band shift.

749 The correlation between $\mathrm{H}_{2} \mathrm{O}$ spectral indicators and $\mathrm{CH}_{4}$ and $\mathrm{N}_{2}$ integrated band depths is 750 explored in Figure 19.

$751 \mathrm{CH}_{4}$ integrated band depths in Ranges 1, 2, and 4 (Figure 19a-f) show a moderate anti752 correlation with the crystalline $\mathrm{H}_{2} \mathrm{O}$ areal mass fraction (panels a, b, c), and a strong anti- 
753 correlation with the $\mathrm{H}_{2} \mathrm{O}$ integrated band depth (panels d, e, f). Pearson correlation coefficient

754 ranges between -0.90 and -0.96 for the plots on the right panels.

755 The correlation is less strong for the integrated band depths of $\mathrm{CH}_{4}$ in Range 3 and at 1.48

$756 \mu \mathrm{m}$, and $\mathrm{N}_{2}$ at $2.15 \mu \mathrm{m}$. In the three left panels of Figure 19 (panels g, h, i), the Pearson

757 coefficient's values are in the interval -0.26 to -0.33 , while in the panels on the right $(j, k, 1)$ the

758 parameter ranges between -0.06 and -0.78 .

759 The geologic units having $\mathrm{H}_{2} \mathrm{O}$ ice are the same forming group $\mathrm{G} 2$ in Figure 13a, or placed on

760 the left side of group G1, characterized by low integrated band depths and low shift index values.

761 The negative correlation observed in Figure 19a-f suggests that $\mathrm{H}_{2} \mathrm{O}$ ice can contribute to the

762 suppression of the strong $\mathrm{CH}_{4}$ ice absorptions and can explain the anomalous trend observed in

763 Figure 13a. For some units, though, $\mathrm{H}_{2} \mathrm{O}$ is neither the only, nor the main, contributor.

764 Unit drt in Al-Idrisi and Baret Montes has the strongest absorptions in the $2.0 \mu \mathrm{m}$ region, but

765 with moderate fractions of crystalline $\mathrm{H}_{2} \mathrm{O}$ ice (28.5\% and $18.1 \%$ respectively). The same is

766 observed for unit abm in Zheng He and Hillary Montes. The discrepancy between the

767 crystalline $\mathrm{H}_{2} \mathrm{O}$ areal mass function and the $\mathrm{H}_{2} \mathrm{O}$ integrated band depths suggests that

768 crystalline $\mathrm{H}_{2} \mathrm{O}$ is contributing to the absorption around $2.0 \mu \mathrm{m}$, but the influence of some other

769 material must be invoked to justify such deep absorptions in this spectral area. Because $\mathrm{CH}_{4}$

770 integrated band depths are also very suppressed, we can deduce that $\mathrm{H}_{2} \mathrm{O}$ and other contaminants

771 are influencing the $\mathrm{CH}_{4}$ absorptions in Ranges 1, 2, and 4 as well. Previous work (e.g.

772 Cruikshank et al., 2015; Schmitt et al., 2017; Protopapa et al., 2017) has noticed a correlation

773 between $\mathrm{H}_{2} \mathrm{O}$ ice and tholins on Pluto, which in Sputnik Planitia is seen at the low-latitude

774 mountain ranges of western Sputnik Planitia. It is therefore likely that tholins, or other material

775 with a positive spectral slope, is significantly contributing to the suppression of $\mathrm{CH}_{4}$ 
776 absorptions in Ranges 1, 2, and 4 in units like drt of Baret and Al-Idrisi Montes, and abm in

777 Zheng He and Hillary Montes.

778 Unit drt in Hillary Montes shows weaker $\mathrm{H}_{2} \mathrm{O}$ integrated band depth than the drt units in the

779 other two provinces, but crystalline $\mathrm{H}_{2} \mathrm{O}$ accounts for $22.6 \%$ of the total composition. The area of

780 unit drt in Hillary Montes is less extensive than the units in Baret and Al-Idrisi Montes, and it is

781 surrounded by units abm and ibm. Unit abm in Baret, Hillary and Zheng He Montes has the

782 highest percentage of crystalline $\mathrm{H}_{2} \mathrm{O}$ and significant $\mathrm{H}_{2} \mathrm{O}$ absorption.

783 The amount of $\mathrm{H}_{2} \mathrm{O}$ ice decreases moving south from the northwestern mountain ranges. Unit

784 ibm shows a lower, but still considerable, amount of $\mathrm{H}_{2} \mathrm{O}$ ice than abm. The lowest abundance of

$785 \mathrm{H}_{2} \mathrm{O}$ ice is seen in units lpp in Hillary Montes, abm and ibm in Tenzing Montes, lpp in Al-Idrisi

786 Montes, and pmp and abm in Southwest buffer. These regions also show medium to low CO

787 content, with the exception of unit abm in Southwest buffer, for which the CO integrated band

788 depth has the deepest value (Figures 15 and 16).

804 positive spectral slope and low albedo, named Cthulhu Macula (Buie et al., 2010; Grundy et al.,

805 2016), the existence of which was also inferred from previous ground-based observations

806 (Cruikshank et al., 2015). This material also covers the low-latitude mountain ranges of western 
807 Sputnik Planitia, seen here in units drt, abm and, in smaller amounts, ibm (Schmitt et al., 2017).

808 This material is interpreted to be tholins, organic compounds that assume colorations in the

809 visible spectrum - from yellow, to red, to brown - when bombarded with high-energy electrons

810 or UV radiation. Tholins don't have a clear spectral feature in the NIR range, but they can

811 weaken the $\mathrm{CH}_{4}$ absorption band at $2.32 \mu \mathrm{m}$, within Range 4 (Schmitt et al., 2017), when the

812 two are mixed together. Comparing the spectrum of Cthulhu, rich in tholins, with that of Kiladze,

813 an impact crater within East Tombaugh Regio where $\mathrm{H}_{2} \mathrm{O}$ is pure and abundant (Cook et al.,

814 2019), shows that around $2 \mu \mathrm{m}$ they have similar shapes. Therefore, disentangling the

815 contribution to the resulting spectrum of $\mathrm{H}_{2} \mathrm{O}$ from $\mathrm{CH}_{4}$ and negative spectral slope material is

816 not easy.

817 Figures 20A and 20B show the distribution of areal mass fraction and grain size of the NSS

818 endmember across Sputnik Planitia.

819 The NSS endmember occurs mainly in units ibm, bct, drt of Al-Idrisi Montes, abm, ibm, dmp

820 and drt of Baret Montes, abm, ibm, dmp, drt and spp of Hillary Montes, pmp and spp of

821 Southwest buffer, and ibm and pmp of Tenzing Montes. The abundance ranges between $14 \%$ and

$82238.5 \%$. In the northern regions of Sputnik Planitia, the NSS endmember accounts for $38.4 \%$ in

823 unit tbp and $26.9 \%$ in dcp, while in the east, this endmember composes between $20.7 \%$ and

$82433.2 \%$ of the sh units in the Colles provinces and the dpp and pmp units.

825 In the units where the NSS endmember is present, we observe a moderate negative trend of

826 the abundance of this component with the integrated band depths of $\mathrm{CH}_{4}$ in Range $1(\rho=-0.34)$,

827 Range $2(\rho=-0.48)$, and Range $4(\rho=-0.55$; Figure 21a). The NSS endmember is not correlated

828 with the $\mathrm{CH}_{4}$ in Range $3(\rho=-0.0007)$, while it has a moderate and slight anti-correlation with 
829 the $\mathrm{CH}_{4}$ band at $1.48 \mu \mathrm{m}(\rho=-0.35)$ and the $\mathrm{N}_{2}$ absorption at $2.15 \mu \mathrm{m}(\rho=-0.28$; Figure $21 \mathrm{~b})$,

830 respectively.

831 Unit drt in Baret and Al-Idrisi Montes hosts the highest amount of NSS endmember, followed

832 by unit abm in Baret and Zheng He Montes, unit tbp in the Cellular plains, units dmp, drt and lpp

833 in Hillary Montes, and unit dmp in Baret Montes. Unit drt and unit abm of Baret Montes also

834 have very suppressed absorptions in the $\mathrm{CH}_{4}$ Ranges 1, 2 and 4, the integrated band depth being

835 close to 0.0. The same suppressed absorptions are also observed in Figure $21 \mathrm{~b}$ for the $\mathrm{CH}_{4}$

836 absorption at $1.48 \mu \mathrm{m}$, and for the $\mathrm{N}_{2}$ feature.

837 Both Figures 21a and 21b do not show a north-south or east-west trend. Units belonging to

838 provinces in the east and south of Sputnik Planitia share similar NSS endmember percentages,

839 and analogous $\mathrm{CH}_{4}$ integrated band depth values.

840 In Figure 22, the abundance of the NSS endmember is compared to the $\mathrm{CH}_{4}$ shift index (top

841 panel), the integrated band depth of $\mathrm{H}_{2} \mathrm{O}$ ice (central panel), the areal mass fraction of crystalline

$842 \quad \mathrm{H}_{2} \mathrm{O}$ ice (bottom panel).

843 The correlation coefficient of the top panel is equal to -0.36 , denoting that a slight dependence

844 between the shift value and the spectra's negative slope exists. In the other two panels, the

845 represented quantities do not show any clear correlation. Indeed, if we do not consider units drt

846 in the middle and bottom panels, the correlation coefficient drops close to 0 . The trend in the

847 middle panel of Figure 22 confirms the difficulty to disentangle $\mathrm{H}_{2} \mathrm{O}$ from tholins in the

848 spectrum, and the necessity to use a radiative transfer model.

849 Assuming that variations in scattering properties do not yield significant changes in the

850 modeled parameters, we can conclude that tholins are primarily responsible for the suppression

851 of $\mathrm{CH}_{4}$ absorptions in Ranges 1,2, and 4 in the units located on the west provinces of Sputnik

* Some feature names mentioned in this paper are now formalized while others are informal 
852 Planitia. For some of these units, like drt in Baret and Al-Idrisi Montes and abm in Baret Montes,

853 tholins are the major contributors of the observed trend of Figure 13a. For the other units in the

854 same area, tholins and $\mathrm{H}_{2} \mathrm{O}$ ice both play a role in suppressing $\mathrm{CH}_{4}$ signatures. Tholins and $\mathrm{H}_{2} \mathrm{O}$

855 ice do not affect the shape and depth of $\mathrm{CH}_{4}$ absorption in Range 3 and of $\mathrm{N}_{2}$ feature at $2.15 \mu \mathrm{m}$.

856 Fig. 20: Maps of variation of NSS endmember areal mass fraction (A) and grain size (B) estimated by the spectral modeling.

857

In our analysis, we examined several refractory materials that are featureless (i.e. they show no

871 range we have examined (Figure 4). The use of kaolinite, a phyllosilicate, in our models consistently

872 returned better fits to the data than any other material, including Plutonian tholins.

In general, hydrated materials display bands at around 1.4 and $1.9 \mu \mathrm{m}$ due to $\mathrm{OH}$ stretch, with the

874 latter band being the stronger of the two. Because the optical constants for diluted $\mathrm{CH}_{4}$ in $\mathrm{N}_{2}$ are

875 highly uncertain in the 1.82-2.11 $\mu \mathrm{m}$ range, we have excluded this band from our work and therefore

876 we cannot fully assess the presence (or absence) of a hydration band around $1.9 \mu \mathrm{m}$. The weaker

877 band around $1.4 \mu \mathrm{m}$, which is in a spectral window that is analyzed in this work, is blended with

878 several weak $\mathrm{CH}_{4}$ bands.

879 Kaolinite also has an identifiable band around $2.3 \mu \mathrm{m}$, but like the $1.9 \mu \mathrm{m}$ region, it is excluded

880 from our analysis due to uncertain optical constants of diluted $\mathrm{CH}_{4}$ in $\mathrm{N}_{2}$. Thus, we cannot confirm if

881 kaolinite specifically is present or not, but rather it likely shares spectral properties with what is

882 observed - a negative spectral slope. 
Tholins produced in Pluto's atmosphere and on Pluto's surface are a likely candidate for the

884 negative spectral slope material on its surface. Tholins, which are typically red at visible

885 wavelengths, can have a slope in the near-infrared spectral region that ranges from positive to negative. The fact that studies of the chemistry of Pluto's atmosphere (e.g. Bertrand \& Forget, 2017;

887 Cheng et al., 2017; Gladstone et al., 2016) produce tholins with a negative spectral slope at near-

888 infrared wavelengths supports the idea that the negative spectral slope material present on Pluto is

889 caused by tholins. To date, however, the optical constants for Plutonian tholins are limited, and their

890 use in our analysis returns non-physical solutions.

891 As explained in Section 3.3, we selected four geologic units rich in red material to test the

892 Plutonian tholins introduced by Cruikshank et al. (2016): unit drt in Al-Idrisi, Hillary, and Baret

893 Montes, and sh in Columbia Colles. Figure 23 shows the LEISA average spectra superimposed upon

894 the best fit model obtained by considering Plutonian tholins as a NSS endmember. Table 5 shows

895 compositional details resulting from the modeling.

896 When Plutonian tholin is used as the NSS endmember, we typically find very small grain sizes

897 (Table 5) and the Hapke code reaches the small grain limit for pure Hapke theory (0.8 $\mu$ m diameter).

898 The code, though, does not jump to the Rayleigh scattering regime (which accounts for particles

899 smaller than 1 micron), likely because that would increase $\chi^{2}$. We also commonly find that the best fit

900 temperature and dilution are increased, sometimes to the limits of our search space (maximum $60 \mathrm{~K}$

901 and 20\% dilution $\mathrm{CH}_{4}$ in $\mathrm{N}_{2}$ ).

902 Specifically comparing models where the NSS endmember is kaolinite or the Plutonian tholin,

903 those models with kaolinite had $\chi 2$ values about a half that of models with Plutonian tholin.

904 These findings lead us to conclude that a surface component with a negative spectral slope must

905 be present in the Sputnik Planitia regions we have examined, and which is perhaps uniformly present

906 on Pluto, but our current understanding of Plutonian tholins is not sufficient to match the

907 observations, and so more laboratory-based spectroscopic measurements are required. 
908

909

910

911

912

913

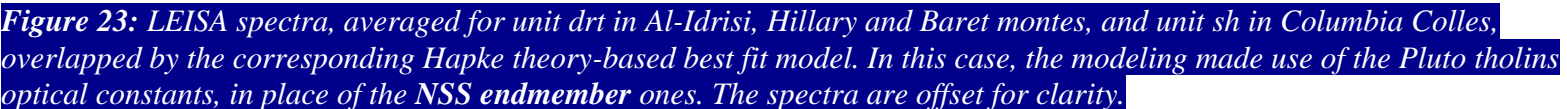

optical constants, in place of the NSS endmember ones. The spectra are offset for clarity.

Table 5: Parameters used to model four LEISA spectra with a modified version of the Hapke theory (i.e. Hapke 1993, 2012), by using Pluto tholins.

\begin{tabular}{|c|c|c|c|c|c|c|c|c|}
\hline Province* & $\mathrm{T}(\mathrm{K})$ & $\begin{array}{c}\mathrm{CH}_{4} \\
\text { fraction } \\
(\%)\end{array}$ & Component & Unit & $\begin{array}{c}\text { Mass } \\
\text { fraction }\end{array}$ & $\begin{array}{c}\text { Grain } \\
\text { diameter } \\
(\mathrm{mm})\end{array}$ & $\begin{array}{c}\text { Fractional } \\
\text { Area }\end{array}$ & $\begin{array}{c}\text { Areal } \\
\text { mass } \\
\text { fraction } \\
(\%)\end{array}$ \\
\hline
\end{tabular}

DRT

\begin{tabular}{|c|c|c|c|c|c|c|c|c|}
\hline \multirow{4}{*}{ Baret Montes } & \multirow{4}{*}{44} & \multirow{4}{*}{11.1} & $\mathrm{~N}_{2}: \mathrm{CH}_{4}$ & A & 1.000 & 32.1 & 0.159 & 15.9 \\
\hline & & & Pluto tholin & & 0.039 & 0.0008 & \multirow{3}{*}{0.841} & 3.3 \\
\hline & & & Crystalline $\mathrm{H}_{2} \mathrm{O}$ & B & 0.802 & 0.093 & & 67.4 \\
\hline & & & Amorphous $\mathrm{H}_{2} \mathrm{O}$ & & 0.159 & 0.003 & & 13.4 \\
\hline \multirow{2}{*}{$\begin{array}{l}\text { Hillary } \\
\text { Montes }\end{array}$} & \multirow{2}{*}{44} & \multirow{2}{*}{0.70} & $\begin{array}{c}\text { pure } \mathrm{CH}_{4} \\
\mathrm{~N}_{2}: \mathrm{CH}_{4}\end{array}$ & A & $\begin{array}{l}0.001 \\
0.999\end{array}$ & $\begin{array}{c}0.58 \\
1,464.2\end{array}$ & 0.625 & $\begin{array}{c}0.1 \\
62.4\end{array}$ \\
\hline & & & $\begin{array}{c}\text { Pluto tholin } \\
\text { Crystalline } \mathrm{H}_{2} \mathrm{O}\end{array}$ & B & $\begin{array}{c}2.0^{*} * 10^{-05} \\
1.000\end{array}$ & $\begin{array}{c}0.59 \\
0.008\end{array}$ & 0.375 & $\begin{array}{c}0.0 \\
37.5\end{array}$ \\
\hline \multirow[b]{2}{*}{$\begin{array}{l}\text { Al-Idrisi } \\
\text { Montes }\end{array}$} & \multirow[b]{2}{*}{49} & \multirow[b]{2}{*}{2.9} & $\mathrm{~N}_{2}: \mathrm{CH}_{4}$ & A & 1.000 & 18.8 & 0.192 & 19.2 \\
\hline & & & $\begin{array}{c}\text { Pluto tholin } \\
\text { Crystalline } \mathrm{H}_{2} \mathrm{O} \\
\text { Amorphous } \mathrm{H}_{2} \mathrm{O}\end{array}$ & B & $\begin{array}{l}0.025 \\
0.384 \\
0.590\end{array}$ & $\begin{array}{c}8.0 * 10^{-4} \\
0.072 \\
0.010\end{array}$ & 0.808 & $\begin{array}{c}2.1 \\
31.0 \\
47.7\end{array}$ \\
\hline \multicolumn{9}{|c|}{ SH } \\
\hline \multirow{2}{*}{$\begin{array}{l}\text { Columbia } \\
\text { Colles }\end{array}$} & \multirow[t]{2}{*}{60} & \multirow[t]{2}{*}{19.1} & $\begin{array}{c}\text { pure } \mathrm{CH}_{4} \\
\mathrm{~N}_{2}: \mathrm{CH}_{4} \\
\beta-\mathrm{N}_{2}\end{array}$ & A & $\begin{array}{l}0.002 \\
0.028 \\
0.970\end{array}$ & $\begin{array}{c}0.13 \\
5.96 \\
634.9 \\
\end{array}$ & 0.573 & $\begin{array}{l}0.1 \\
1.6 \\
55.6\end{array}$ \\
\hline & & & $\begin{array}{c}\text { Pluto tholin } \\
\text { Crystalline } \mathrm{H}_{2} \mathrm{O}\end{array}$ & B & $\begin{array}{l}0.008 \\
0.992\end{array}$ & $\begin{array}{c}8.0 * 10^{-4} \\
0.038\end{array}$ & 0.427 & $\begin{array}{c}0.4 \\
42.3\end{array}$ \\
\hline
\end{tabular}

914

$915 \quad$ 5. Correlation of spectral results and observed geology

$916 \quad 5.1$ Blocky mountains and inter-block material.

917 The chaotic, angular, blocky mountains (unit abm), form a discontinuous buffer between

918 Sputnik Planitia and the uplands to the west. The mountain blocks typically reach $>10 \mathrm{~km}$ in

919 diameter and are mostly contained within the ranges of Al-Idrisi, Baret, Hillary, Tenzing, and

920 Zheng He Montes. Isolated mountain blocks are also seen within the dcp unit of the Cellular 
921 plains, the largest being Coleda de Dados Colles. The inter-block material (unit ibm) consists of

922 chaotically oriented, close-packed blocks, typically reaching a few to several km across, that

923 occur interstitially to and can also embay the abm unit. The blocks are similar to, but smaller

924 than, the blocks forming the abm unit. The finer texture of unit ibm compared to abm may

925 indicate breaking up of mountain blocks into smaller fragments and/or size filtering thereafter

926 (White et al., 2017; O’Hara \& Dombard, 2020). The mountain blocks that form these units are

927 randomly oriented and have been hypothesized to consist of fragments of the $\mathrm{H}_{2} \mathrm{O}$ ice crust that

928 have been detached by tectonism and intruded, transported, and rotated by glacial $\mathrm{N}_{2}$ ice (White

929 et al., 2017).

930 Crystalline $\mathrm{H}_{2} \mathrm{O}$ ice is indeed present to various degrees in both units, with areal mass

931 fractions ranging between $9.3 \%$ and $47.1 \%$ (Figure $17 \mathrm{a}$ ). The abundance of crystalline $\mathrm{H}_{2} \mathrm{O}$ ice

932 within unit abm is broadly constant between Al-Idrisi, Baret, and Hillary Montes (varying

933 between $35.4 \%$ and $41.9 \%$ ), although there is a dip to $25.1 \%$ at Zheng-He Montes. To the south

934 of Hillary Montes, there is a substantial decrease in the abundance from $18.3 \%$ at the Southwest

935 buffer to $9.3 \%$ at Tenzing Montes. The abundance of NSS endmember within abm reaches a

936 maximum in the low northern latitudes: Al-Idrisi Montes shows none, Zheng He, Baret and

937 Hillary Montes show 41.6\%, 36.7\%, and 27.8\% respectively, and the Southwest buffer and

938 Tenzing Montes show none. The NSS endmember maximum in the central Montes correlates to

939 the occurrence of dark tholins coating Zheng He, Baret and Hillary Montes, which unlike Al-

940 Idrisi and Tenzing Montes are co-latitudinal with the equatorial maculae that extend in a

941 discontinuous belt around Pluto. The maculae are interpreted to represent tholins that have

942 formed via ultraviolet irradiation of $\mathrm{CH}_{4}$ in Pluto's atmosphere, settled out onto the surface, and

943 accumulated to form a superficial, but contiguous, layer (Moore et al., 2016; Grundy et al., 
944 2018). This process occurs across Pluto's entire surface, but the low latitude uplands within the

945 permanent diurnal zone are the only location where climatic conditions are mild enough on

946 obliquity timescales such that the layer is not dissipated by regular volatile mobilization (Binzel

947 et al., 2017; Earle et al., 2017, 2018). Grundy et al. (2018) estimated that unperturbed tholin

948 accumulation would coat the surface to a thickness of $\sim 3.5 \mathrm{~m}$ over $1 \mathrm{Gyr}$, a result supported by

949 Johnson et al. (2020). The elevated abundance of NSS endmember within the abm unit of the

950 central Sputnik mountain ranges, in combination with the observation that they are coated by a

951 dark material whereas peaks in the northern and southern ranges are not, therefore indicates that

952 these dislodged crustal blocks are sufficiently stable in their grounded state, surrounded by $\mathrm{N}_{2}$

953 ice of Sputnik Planitia, to accumulate tholins to the extent that they form a contiguous layer over

954 millions of years. As noted in Section 4, it is difficult to disentangle the effects of $\mathrm{H}_{2} \mathrm{O}$ ice and

955 negative spectral slope material at around $2 \mu \mathrm{m}$, and previous works (e.g. Cruikshank et al.,

956 2015, Schmitt et al., 2017, Protopapa et al., 2017) have accordingly identified a correlation

957 between the abundance of $\mathrm{H}_{2} \mathrm{O}$ ice and tholins, which may form part of the explanation for the

958 generally high abundance of $\mathrm{H}_{2} \mathrm{O}$ ice within the central Sputnik mountain ranges (especially

959 Baret Montes).

960 For both the abm and ibm units, there is a pronounced minimum in the abundance of volatile

961 ices for the central Sputnik mountain ranges. The areal mass fraction of $\mathrm{N}_{2}: \mathrm{CH}_{4}$ and $\beta-\mathrm{N}_{2}$ in abm

962 is $64.6 \%$ at Al-Idrisi, which dips to $33.3 \%$ and $21.4 \%$ in Zheng He and Baret Montes

963 respectively, before increasing to $34.7 \%$ in Hillary Montes, $81.3 \%$ in the Southwest buffer, and

$96489.3 \%$ at Tenzing Montes. We interpret this pattern to be a consequence of the extensive tholin

965 coverage in the central ranges, and it is notable that it is anti-correlated to the NSS endmember,

966 the abundance of which reaches a maximum in these ranges. For those ranges not coated with 
967 tholins, the elevated abundance of volatiles in the southern mountain ranges (81.3-89.3\%)

968 relative to those in the north $(64.6 \%)$ is interpreted to be a consequence of the highly variable

969 insolation and climate zones across the latitudinal extent of Sputnik Planitia that derive from

970 Pluto's high obliquity (presently $123^{\circ}$ ) (Hamilton et al., 2016; Binzel et al., 2017; Earle et al.,

971 2017). Modeling of the $\mathrm{N}_{2}$ cycles on Pluto over seasonal and astronomical timescales by

972 Bertrand et al. (2018) shows that over one obliquity cycle in Pluto's current orbital configuration,

973 the latitudes of Sputnik Planitia $\left(25^{\circ} \mathrm{S}-30^{\circ} \mathrm{N}\right)$ are dominated by $\mathrm{N}_{2}$ condensation, entirely

974 incorporating Tenzing Montes between $9^{\circ} \mathrm{S}$ and $22^{\circ} \mathrm{S}$, whereas the northern regions between

$97530^{\circ} \mathrm{N}$ and $50^{\circ} \mathrm{N}$ are dominated by $\mathrm{N}_{2}$ sublimation, mostly incorporating Al-Idrisi Montes

976 between $\left(25^{\circ} \mathrm{N}-42^{\circ} \mathrm{N}\right)$. This effect may explain the paucity of interstitial $\mathrm{N}_{2}$ ice separating the

977 mountain blocks of northern ranges such as Al-Idrisi (Fig. 25a), relative to the blocks of Tenzing

978 Montes, the flanks of which appear to be inundated by $\mathrm{N}_{2}$ ice plains that are elevated by as much

979 as a kilometer above those of Sputnik Planitia (Fig. 25b), implying voluminous $\mathrm{N}_{2}$ ice

980 accumulation amongst the blocks here. The extensive coating of the southern mountains by such

981 condensed volatiles likely explains the lower detections of $\mathrm{H}_{2} \mathrm{O}$ ice here relative to the northern

982 mountains, where the $\mathrm{H}_{2} \mathrm{O}$ ice is more exposed. Volatile condensation is especially enhanced in

983 southern Sputnik at present as these regions are entering winter, whereas the northern regions are

984 entering summer.

985 A similar effect may be seen in the areal mass fraction of pure $\mathrm{CH}_{4}$ ice in unit abm, which

986 while forming less than a few percent of this unit, shows a general increase from north to south:

987 it is absent at Al-Idrisi, Zheng He, Baret, and Hillary Montes, increases to $0.4 \%$ at the Southwest

988 buffer, and to $1.4 \%$ at Tenzing Montes (the highest areal mass fraction for any unit in any

989 province in the mapping area). For the latter range, $\mathrm{N}_{2}$ absorption is especially suppressed (Fig. 
990 10). $\mathrm{N}_{2}$ and $\mathrm{CH}_{4}$ will both deposit at low elevations, but the higher abundance of $\mathrm{N}_{2}$ relative to

$991 \mathrm{CH}_{4}$ in Pluto's atmosphere, in combination with the warmer atmosphere at high elevations

992 inhibiting N2 precipitation (Moore et al., 2018; Bertrand et al., 2020a), means that deposition of

$993 \mathrm{~N}_{2}$ dominates at lower elevations, whereas $\mathrm{CH}_{4}$ is mostly deposited at high elevation. Therefore,

994 both the very high elevation of Tenzing Montes (rising to $>5 \mathrm{~km}$ above mean radius) and their

995 equatorial latitude make them a natural setting for the precipitation of $\mathrm{CH}_{4}$ ice under Pluto's

996 present obliquity and seasonal conditions. The volatile transport modeling of Bertrand et al.

997 (2019) found that at higher latitudes, $\mathrm{CH}_{4}$ deposits are only stable on a seasonal timescale for

998 Pluto's current orbital configuration, but at lower latitudes, such deposits at high elevation would

999 grow thicker and potentially subsist across multiple Pluto years, as in the case of the bright $\mathrm{CH}_{4}$

1000 deposits that are seen to coat the summits of Pigafetta and Elcano Montes within Cthulhu

1001 Macula, the crests of which rise to almost $4 \mathrm{~km}$ above mean radius (Bertrand et al., 2020a). The

$1002 \mathrm{CH}_{4}$ at Tenzing Montes is not characterized by a bright coating unlike the summits of the co-

1003 latitudinal Pigafetta and Elcano Montes, which may be due to (i) the overall low abundance of

1004 pure $\mathrm{CH}_{4}$ ice here (1.4\%), (ii) the lower albedo contrast between $\mathrm{CH}_{4}$ frost and $\mathrm{H}_{2} \mathrm{O}$ ice as

1005 compared to $\mathrm{CH}_{4}$ frost and the dark blanket of tholins, and (iii) perhaps also the fact that Tenzing

1006 Montes were seen at a very high solar incidence angle $\left(\sim 77^{\circ}\right)$ during the New Horizons flyby,

1007 which minimizes the visibility of the albedo contrast between the $\mathrm{CH}_{4}$ frost and $\mathrm{H}_{2} \mathrm{O}$ ice.

1008

$1009 \quad 5.2$ Bright chaotic terrain

1010 The bright, chaotic terrain unit (bct) is confined to a single region to the north of Al-Idrisi

1011 Montes. It consists of high albedo plains interspersed with darker blocks reaching several km

1012 across. The unit is crossed by east-west oriented troughs and scarps, and is at a slightly lower

* Some feature names mentioned in this paper are now formalized while others are informal 
1013 elevation (by $\sim 0.5 \mathrm{~km}$ ) than unit ibm to the south, making it amongst the lowest elevation

1014 regions of anywhere in Sputnik Planitia and its borders (reaching $3 \mathrm{~km}$ below mean radius). The

1015 fact that the unit displays a geomorphology that is more characteristic of the fluted, valleyed

1016 uplands to the north led White et al. (2017) to hypothesize that it may represent an early stage in

1017 the formation of the chaotic, angular, blocky mountains, whereby tectonically weakened $\mathrm{H}_{2} \mathrm{O}$ ice

1018 crust is in the process of fragmenting as it is being infiltrated by glacial $\mathrm{N}_{2}$ ice that has

1019 overflowed from Sputnik Planitia, or condensed directly onto it from the atmosphere in large

1020 quantities on account of the terrain's very low elevation. This hypothesis is supported by the

1021 composition of bct, very similar to the ibm unit, which is interpreted to represent small $\mathrm{H}_{2} \mathrm{O}$ ice

1022 crustal blocks that have been dislodged and coated by the glacial $\mathrm{N}_{2}$ ice. $\beta-\mathrm{N}_{2}$ is the most

1023 abundant component in bct and ibm (54.8\% and $41.2 \%$ respectively), followed by crystalline

$1024 \mathrm{H}_{2} \mathrm{O}$ ice (25.5\% and 34.9\%), the NSS endmember (14\% and 21.3\%), and $\mathrm{N}_{2}: \mathrm{CH}_{4}(5.7 \%$ and

$10252.5 \%$ ). The abundance of $\mathrm{N}_{2}$ in this area is also confirmed by LEISA spectra: Figures 6 and 10

1026 show that unit bct has the highest $\mathrm{CH}_{4}$ band shift parameter and deepest $\mathrm{N}_{2}$ absorption band of

1027 any unit.

1028

$1029 \quad 5.3$ Dark, Ridged Terrain

1030 The dark, ridged terrain (unit drt) consists of low albedo, subparallel ridges with crest-to-crest

1031 spacing of several hundred meters to a few km, which mainly separate Baret and Al-Idrisi

1032 Montes from the uplands of Viking Terra to the west, although a smaller occurrence is also seen

1033 separating Hillary Montes from the dark-pitted, marginal plains. The ridges tend to align parallel

1034 to the edge of Sputnik Planitia. Unit drt displays the highest areal mass fraction of the NSS

1035 endmember of any unit (38.5\%-53.4\%), followed by $\mathrm{N}_{2}: \mathrm{CH}_{4}(21.5 \%-38.7 \%)$, and $\mathrm{H}_{2} \mathrm{O}$ ice 
$1036(18.1 \%-28.5 \%)$. The high abundance of NSS endmember reflects the fact that this unit displays

1037 the most contiguous and complete coverage by tholins of any unit. The ridges have been

1038 variously interpreted to be compressional in origin, whereby they form due to buoyant, mobile

$1039 \mathrm{H}_{2} \mathrm{O}$ ice blocks being pushed towards the uplands by convective motions in Sputnik Planitia,

1040 with a weaker, darker, overlying layer folding in response to the compressive forces (White et

1041 al., 2017; Ahrens \& Chevrier, 2019), or depositional, whereby they are recessional moraines of

1042 dark tholins deposited as the $\mathrm{N}_{2}$ ice glaciers responsible for entraining and translating the $\mathrm{H}_{2} \mathrm{O}$

1043 ice mountains retreated (Howard et al., 2017). White et al. (2017) hypothesized that this dark

1044 material represents tholins like those coating Cthulhu Macula and Baret, Zheng He, and Hillary

1045 Montes, while Ahrens and Chevrier (2019) interpreted the ridges to be formed from a thin layer

1046 of $\mathrm{CH}_{4}-\mathrm{H}_{2} \mathrm{O}$ ice overlain on a $\mathrm{H}_{2} \mathrm{O}$ ice-based glacier. Our spectral analysis and modeling does

1047 not suggest that the dark material is especially enriched in $\mathrm{CH}_{4}$, with between $0.5 \%$ and $0.8 \%$

$1048 \mathrm{CH}_{4}$ being diluted in the $\mathrm{N}_{2}: \mathrm{CH}_{4}$ (Fig. 8A) that forms between $21.5 \%$ and $38.7 \%$ of the unit by

1049 areal mass fraction. The prevalence of the NSS endmember that characterizes this unit, and its

1050 very low albedo, leads us to conclude that the deformed layer is more likely composed of tholins

1051 rather than a $\mathrm{CH}_{4}-\mathrm{H}_{2} \mathrm{O}$ ice mixture.

1052 There is always a sharp and well-defined boundary between unit drt and adjoining units - the

1053 dark tholins are always tightly confined to within the unit's boundaries and do not spread beyond

1054 its borders, implying that whatever process has created this unit, it has acted to densely

1055 concentrate the tholins within the unit. In addition, whereas the drt units of Baret and Hillary

1056 Montes are mostly located within the equatorial, permanent diurnal zone $\left(13^{\circ} \mathrm{N}\right.$ to $\left.13^{\circ} \mathrm{S}\right)$ that

1057 harbors the maculae and the tholin-covered mountain ranges, that of Al-Idrisi Montes extends to

1058 higher latitudes $\left(20^{\circ} \mathrm{N}\right.$ to $\left.30^{\circ} \mathrm{N}\right)$, suggesting that accumulation directly via settling from the 
1059 atmosphere alone cannot explain the density of tholins here, as it can for the tholin coverage on

1060 the mountain ranges. Since the low albedo $\mathrm{N}_{2}$ ice plains of northern Sputnik Planitia

1061 (encompassing units dcp and tbp, see section 5.5) are interpreted to have high levels of tholins

1062 entrained within them, the same compressive forces that White et al. (2017) and Ahrens and

1063 Chevrier (2019) hypothesized formed the ridges may have also concentrated tholins within $\mathrm{N}_{2}$

1064 ice separating the mountain blocks, dredged them to the surface, and formed them into the sub-

1065 parallel ridges. We further discuss unit drt in section 5.4, in which we argue that Columbia

1066 Colles, mapped by White et al. (2017) as scattered hills, displays geomorphological and spectral

1067 characteristics associated with unit drt.

1068

\subsection{Scattered hills}

1070 The scattered hills form a unit (sh) in eastern Sputnik Planitia consisting of km-sized hills

1071 with up to $500 \mathrm{~m}$ topographic relief that have been carried by $\mathrm{N}_{2}$ ice valley glaciers flowing from

1072 East Tombaugh Regio into the Cellular plains of Sputnik Planitia (White et al., 2017). In

1073 addition, there are Challenger and Columbia Colles, larger conglomerations of such hills that

1074 form roughly rectangular masses measuring several tens of $\mathrm{km}$ across, and which have been

1075 interpreted to be grounded in shallow $\mathrm{N}_{2}$ ice here at the edge of Sputnik basin (White et al.,

1076 2017). Given that these hills appear to be subject to the motions of $\mathrm{N}_{2}$ ice (both glacial and

1077 convective) in this region of Sputnik Planitia, White et al. (2017) and Howard et al. (2017)

1078 hypothesized that they represent chunks of the pitted uplands that have been broken off through

1079 glacial erosion and transported in the $\mathrm{N}_{2}$ ice. Geologic mapping of the pitted uplands and the

$1080 \mathrm{CH}_{4}$ ice bladed terrain of Tartarus Dorsa to the east indicates that the pitted uplands are likely a

1081 lower-elevation extension of the bladed terrain that is experiencing deposition of volatile ices 
1082 that have sublimated from Sputnik Planitia (Moore et al., 2018). This would imply that the pitted 1083 uplands, and the scattered hills derived from them, are predominantly composed of $\mathrm{CH}_{4}$ ice.

1084 Our spectral modeling of the discrete ranges of hills that form unit sh shows a relatively 1085 consistent pattern: hills in the South-East pitted plains and Astrid, Challenger, and Soyuz Colles 1086 show a composition that is predominantly $\beta-\mathrm{N}_{2}(64.4 \%-79 \%)$, followed by the NSS endmember $1087(19.7 \%-32.4 \%)$, with a small areal mass fraction of $\mathrm{N}_{2}: \mathrm{CH}_{4}(1.3 \%-3.6 \%)$. These hills are located 1088 within the portion of Sputnik Planitia that is interpreted to be experiencing net $\mathrm{N}_{2}$ ice deposition 1089 under Pluto’s current climatic and obliquity conditions (White et al., 2017, Bertrand et al., 2018), 1090 and the dominance of $\beta-\mathrm{N}_{2}$ in the spectra reflects this. In $315 \mathrm{~m} /$ pixel imaging of the hills, they 1091 show a similarly high albedo with respect to the surrounding $\mathrm{N}_{2}$ ice plains, and their surface 1092 relief appears smoothened, indicating that they have accumulated a contiguous coating of $\mathrm{N}_{2}$ ice 1093 (Fig. 26a). This coating is thick enough to obscure the spectral signal from the material 1094 composing the hills themselves, so we cannot confirm spectroscopically whether the hills are 1095 composed of $\mathrm{H}_{2} \mathrm{O}$ or $\mathrm{CH}_{4}$ ice (both of which are less dense than $\mathrm{N}_{2}$ ice, allowing the hills to 1096 float, and both of which are rigid enough at Pluto conditions to support the topographic relief 1097 exhibited by the hills).

1098 A notable exception to the trend is Columbia Colles, which displays a composition that is 1099 more similar to the dark, ridged terrain (unit drt) than the other scattered hills (cf. Fig. 24N to the 1100 drt units in Figs. 24A, B, and F). Like the other scattered hills, Columbia Colles displays 1101 substantial $\beta-\mathrm{N}_{2}(45.5 \%)$ and NSS endmember (21.7\%), with a small areal mass fraction of $1102 \mathrm{~N}_{2}: \mathrm{CH}_{4}(0.05 \%)$, but also displays a large areal mass fraction of crystalline $\mathrm{H}_{2} \mathrm{O}(32.8 \%)$. The $1103 \mathrm{H}_{2} \mathrm{O}$ ice may be attributed to the fact that Columbia Colles are the only portion of the unit sh to 1104 display a consistent covering by dark red tholins, the spectral signature of which, as noted earlier, 
1105 is difficult to distinguish from that of $\mathrm{H}_{2} \mathrm{O}$ ice. Unit drt also shows significant crystalline $\mathrm{H}_{2} \mathrm{O}$

$1106(18.1 \%-28.5 \%)$ and NSS endmember (38.5\%-53.4\%), but with $\mathrm{N}_{2}: \mathrm{CH}_{4}(21.5 \%-38.7 \%)$ in place

1107 of $\beta-\mathrm{N}_{2}$, which is absent. Portions of Columbia Colles display a quite well-developed ridged

1108 fabric, with ridge crests separated by $1-2 \mathrm{~km}$ (very similar to the separation of the ridge crests of

1109 unit drt, Figs. 26b and 26c). White et al. (2017) mapped Columbia Colles as part of unit sh on

1110 account of its proximity to the other scattered hills and close spatial relationship to Challenger

1111 Colles (a discontinuous "bridge" of hills $60 \mathrm{~km}$ long joins the two conglomerations). The

1112 distinct dark red color of Columbia Colles, its more explicitly ridged texture compared to

1113 anything seen elsewhere within unit sh, and the fact that Columbia Colles is the only portion of

1114 unit sh that contacts the surrounding uplands (as do the occurrences of unit drt in Baret and Al-

1115 Idrisi Montes, and possibly also that in Hillary Montes if the dark-pitted, marginal plains that

1116 bound it represent uplands terrain of Cthulhu Macula covered by thin $\mathrm{N}_{2}$ ice), however, are

1117 characteristics that are more diagnostic of unit drt. Unit drt is interpreted to represent an

1118 accumulation of dark tholins superposed on lighter-toned $\mathrm{H}_{2} \mathrm{O}$ ice blocks that have eroded from

1119 Pluto's ancient $\mathrm{H}_{2} \mathrm{O}$ ice crust along the western margin of Sputnik Planitia (section 5.3), whereas

1120 Columbia Colles, and the rest of the scattered hills, are interpreted to consist of blocks of $\mathrm{CH}_{4}$ ice

1121 that were eroded more recently from the pitted uplands of East Tombaugh Regio. Therefore,

1122 while we hypothesize that the same processes have probably caused the concentration of dark

1123 surface material at the western dark ridged terrain as well as Columbia Colles, and its

1124 arrangement into subparallel ridges, the material forming Columbia Colles is still distinct

1125 stratigraphically and compositionally from that forming the western dark ridged terrain, and so

1126 mapping Columbia Colles as unit sh, rather than drt, remains justified. The processes forming the

1127 ridges are not known for certain, but compression of the underlying blocky material and glacial 
1128 recession have both been invoked (Howard et al., 2017; White et al., 2017; Ahrens and Chevrier, 1129 2019).

\subsection{Cellular plains}

1132 The Cellular plains of Sputnik Planitia represent the portion of the $\mathrm{N}_{2}$ ice sheet that is

1133 experiencing solid-state convection from thermal input within or below the glacier (Stern et al.,

1134 2015; Moore et al., 2016; McKinnon et al., 2016; Trowbridge et al., 2016). The plains dominate

1135 the central and northern portions of Sputnik Planitia, i.e. the part of Sputnik Planitia

1136 corresponding to the Sputnik basin itself, where the $\mathrm{N}_{2}$ ice is sufficiently thick $(>1 \mathrm{~km})$ to

1137 support convection (McKinnon et al., 2016). The cells tend to be ovoid in planform, are defined

1138 by a reticulate network of troughs with central ridges, and exhibit pitting on a scale of hundreds

1139 of meters to a few km (Moore et al., 2016; White et al., 2017). The timescale of surface renewal

1140 via convection has been estimated to be $\sim 420,000$ to $\sim 890,000$ years (McKinnon et al., 2016;

1141 Trowbridge et al., 2016; Buhler \& Ingersoll, 2018). White et al. (2017) divided the Cellular

1142 plains into three units based primarily on albedo. The bright, Cellular plains (unit bcp) occupy

1143 the central portion of Sputnik Planitia, extending to $\sim 30^{\circ} \mathrm{N}$. They display a normal reflectance of

11440.78 to 0.86 (Buratti et al., 2017) and are interpreted to be the region of Cellular plains that are

1145 presently experiencing net deposition of fresh, bright $\mathrm{N}_{2}$ frost from the northern regions of

1146 Sputnik Planitia (Protopapa et al. 2017; Bertrand et al., 2018, 2020b). The dark, Cellular plains

1147 (unit dcp, with a normal reflectance of $~ 0.69$ ), and dark, trough-bounding plains (unit tbp, with a 1148 normal reflectance of $\sim 0.52-0.60$ ) occur in the northern and western portions of Sputnik Planitia, 1149 and are interpreted to be presently experiencing net sublimation (Protopapa et al. 2017; Bertrand 
1150 et al., 2018, 2020b). With the exception of Alcyonia Lacus in Al-Idrisi Montes, all three units of

1151 the Cellular plains are confined to the Cellular plains in this study.

1152 Unsurprisingly, the most abundant component of all three units is $\mathrm{N}_{2}$ ice, the $\mathrm{CH}_{4}$ fraction

1153 diluted in $\mathrm{N}_{2}$ always being below $1 \%$. This observation is also confirmed by the high values of

1154 the $\mathrm{CH}_{4}$ band shift index, and by the depth of $\mathrm{N}_{2}$ absorption band. Unit bcp is dominated by

$1155 \mathrm{~N}_{2}: \mathrm{CH}_{4}$, which accounts for $99.9 \%$ of its areal mass fraction, corroborating the interpretation that

1156 the high albedo of unit bcp is due to current deposition of fresh $\mathrm{N}_{2}$ frost on its surface White et

1157 al. (2017). Units dep and tbp are instead dominated by $\beta-\mathrm{N}_{2}$ ice: dep shows $68.2 \% \beta-\mathrm{N}_{2}$ and

$11584.9 \% \mathrm{~N}_{2}: \mathrm{CH}_{4}$, whereas tbp shows $53.9 \% \beta-\mathrm{N}_{2}$ and $7.6 \% \mathrm{~N}_{2}: \mathrm{CH}_{4}$. The remainders of these units

1159 are mainly occupied by NSS endmember: $26.9 \%$ for dcp and $38.4 \%$ for tbp. Based on the results

1160 of Protopapa et al. (2017) and Schmitt et al. (2017), White et al. (2017) interpreted unit dep to be

1161 representative of the material filling the Sputnik basin, specifically $\mathrm{N}_{2}$ ice that is associated with

1162 a relatively high concentration of tholins that first settled onto the surface by katabatic and

1163 condensation flows (Bertrand et al., 2020b) and then became entrained within the ice by

1164 convective motion across the history of the existence of Sputnik Planitia (White et al., 2017).

1165 This $\mathrm{N}_{2} /$ tholin mixture is only exposed in the north and west where the net sublimation of the $\mathrm{N}_{2}$

1166 ice means that it is not blanketed by a fresh layer of bright $\mathrm{N}_{2}$ frost as seen in the rest of Sputnik

1167 Planitia, including unit bcp (Bertrand et al., 2018, 2020b). In the case of the lower albedo unit

1168 tbp, confined to the northern edge of Sputnik Planitia, White et al. (2017) interpreted this to be

1169 where tholins in the $\mathrm{N}_{2}$ ice had become especially concentrated at cell boundaries through a

1170 combination of convective motion of the ice and the very high sublimation rates encountered

1171 here (higher than anywhere else in Sputnik Planitia across an obliquity cycle). In accordance

1172 with this hypothesis, we find that the proportions of NSS endmember within these three Cellular 
1173 plains units correlate to their albedos, and indicate a progressively increasing tholin content 1174 within darker plains.

1175 Alcyonia Lacus is an irregular, $30 \mathrm{~km}$ long by $10 \mathrm{~km}$ wide expanse of $\mathrm{N}_{2}$ ice plains that is 1176 surrounded by units abm and ibm of Al-Idrisi Montes, and which was mapped as dcp by White et 1177 al. (2017) (Fig. 27a). While the plains do show the same albedo as unit dep within Sputnik

1178 Planitia, they are separated from the Cellular plains by the mountain range, so cannot strictly be 1179 described as "cellular". The absence of pits indicates that it is being resurfaced by some means 1180 other than sublimation, and/or that its rheology is unable to support pit topography. Its relatively 1181 smooth boundaries, whereby the surrounding mountains and hills do not intrude into its interior, 1182 indicate that this feature may have actively modified its boundaries. It has variously been 1183 interpreted as once being a "pond" of liquid $\mathrm{N}_{2}$ maintained during an era of high atmospheric 1184 pressure, and which has since frozen (Stern et al., 2017), or an active glacial feature in which 1185 flow of the $\mathrm{N}_{2}$ ice closes any sublimation pits and shepherds blocky $\mathrm{H}_{2} \mathrm{O}$ ice material to its edges 1186 (White et al., 2017). Spectral modeling shows that its composition is broadly similar to that of 1187 the dcp unit proper within Sputnik Planitia, exhibiting 53.9\% $\beta-\mathrm{N}_{2}, 20.7 \%$ NSS endmember, and $11881.9 \% \mathrm{~N}_{2}: \mathrm{CH}_{4}$, but with an additional $\mathrm{H}_{2} \mathrm{O}$ ice component, comprising 23.6\%. Sub-resolution $1189 \mathrm{H}_{2} \mathrm{O}$ ice fragments floating within the $\mathrm{N}_{2}$ ice of the lacus may cause this. The presence of the 1190 NSS endmember suggests that, like unit dcp within the Cellular plains, Alcyonia Lacus also 1191 consists of "mature" $\mathrm{N}_{2}$ ice that has entrained a high concentration of tholins within its volume 1192 over the course of its existence, but given its geographic isolation from Sputnik Planitia (the two 1193 are separated by $65 \mathrm{~km}$ ), it most likely formed independently from Sputnik Planitia and 1194 separately acquired its tholins. White et al. (2017) hypothesized that the lacus may be situated at 1195 a localized zone of high geothermal flux that is mobilizing the $\mathrm{N}_{2}$ ice and causing any blocky 
$1196 \mathrm{H}_{2} \mathrm{O}$ fragments within the zone of mobilization to drift to its edges, and if the lacus is long-lived,

1197 then it is possible that such continual mobilization has also allowed gradual entrainment of

1198 tholins within the $\mathrm{N}_{2}$ ice to levels comparable to that seen within unit dcp of the Cellular plains.

\subsection{Non-Cellular plains}

The non-Cellular plains of Sputnik Planitia are those that are interpreted to not be currently experiencing solid-state convection. These plains dominate the southern and eastern regions of

1203 Sputnik Planitia, but there are also small occurrences seen interstitially to mountain and Cellular

1204 plains units at the western and northern boundaries. As with the Cellular plains, these plains

1205 exhibit pitted textures, but the pits can reach much larger diameters (typically $>1 \mathrm{~km}$ ). The

1206 transition from cellular to non-Cellular plains is interpreted to reflect thinning of the volatile ice

1207 layer, and perhaps also declining heat flow (McKinnon et al., 2016). The non-Cellular plains are 1208 not convecting, but they have nevertheless very recently resurfaced via glacial flow of the $\mathrm{N}_{2}$ ice 1209 from which they are formed, possibly on a timescale as short as tens of thousands of years (Wei

1210 et al., 2018). The non-Cellular plains universally show a high normal reflectance (0.78 to 0.86),

1211 like that of the bright Cellular plains. This is true even for the small patches of non-Cellular

1212 plains located at the northern and western edges of Sputnik Planitia (the North pitted plains and

1213 West pitted plains provinces), which are situated within the portion of Sputnik Planitia that is

1214 currently experiencing net sublimation and which abut the darker plains units (dcp and tbp)

1215 (White et al., 2017; Bertrand et al., 2018, 2020b).

$1216 \quad \beta-\mathrm{N}_{2}$ and $\mathrm{N}_{2}: \mathrm{CH}_{4}$ are the most abundant components of the non-Cellular plains: in the

1217 southern region of Sputnik Planitia, the deeply pitted plains (unit dpp), lightly pitted plains (unit 1218 lpp), sparsely pitted plains (unit spp), patchy, pitted, marginal plains (unit pmp), and dark-pitted 
1219 marginal plains (unit dmp) display between $59.2 \%$ and $100 \% \mathrm{~N}_{2}: \mathrm{CH}_{4}$ and $\beta-\mathrm{N}_{2}$ by areal mass

1220 fraction. The proportion of $\mathrm{N}_{2}: \mathrm{CH}_{4}$ and $\beta-\mathrm{N}_{2}$ versus NSS endmember within these plains appears

1221 to correlate to the extent to which the plains display deep pits. The plains units exhibiting the

1222 largest pits (typically reaching $\sim 1 \mathrm{~km}$ in diameter, but which can reach several $\mathrm{km}$ ) are the

1223 deeply pitted plains, which are primarily concentrated in the southernmost region of Sputnik

1224 Planitia where they dominate the South pitted plains province, and the dark-pitted, marginal

1225 plains, which form a buffer between the mountain ranges of Hillary and Baret Montes and the

1226 uplands of Cthulhu Macula. The pits in both of these units tend to cluster together in tightly

1227 packed swarms that form curved, wavelike patterns (White et al., 2017), and where they are large

1228 enough for their floors to be resolved and not obscured by shadows cast by the pit walls, the

1229 floors invariably display a low albedo ( $<0.17$ normal reflectance, Buratti et al., 2017). This low

1230 albedo material has been interpreted to be tholins covering the pit floors (White et al., 2017),

1231 which in the case of dpp may be tholins entrained within the $\mathrm{N}_{2}$ ice that have been released via

1232 sublimation to build up on the floors of the pits as a lag, and in the case of dmp may be tholins of

1233 Cthulhu Macula, whereby the $\mathrm{N}_{2}$ ice covering the macula is thin enough for the pits to extend all

1234 the way through the ice, exposing the tholins of Cthulhu Macula underneath. Our models show

1235 that both units exhibit NSS endmember: $20.7 \%$ for dpp in the South pitted plains, and $38.9 \%$ to

1236 61.4\% for dmp in Baret and Hillary Montes respectively. The high abundance of NSS

1237 endmember in the latter may be a consequence of the lower solar incidence angle of the imaging

1238 covering unit dmp relative to dpp, meaning that shorter shadows are cast by the pit walls across

1239 pit floors in dmp, and so higher proportions of the pit floors are visible. Pits within dpp in

1240 southern Sputnik Planitia are mostly small enough for shadows to cast across their entire floors. 
1241 In contrast to these plains units that display large and deep pits, the lightly pitted plains and 1242 sparsely pitted plains were interpreted by White et al. (2017) to be experiencing only superficial 1243 sublimation of $\mathrm{N}_{2}$ ice, and are instead undergoing mantling by ongoing deposition of $\mathrm{N}_{2}$ frost, as 1244 well as glacial creep of the $\mathrm{N}_{2}$ ice that is infilling any large pits that do exist. Any sublimation 1245 occurring within these plains is therefore insufficient to counter these processes and to create 1246 large pits that expose tholins entrained within the $\mathrm{N}_{2}$ ice, meaning that neither of these units is 1247 interpreted to display non-volatile components (i.e. NSS endmember) in their composition.

1248 Accordingly, both of these units are dominated by $\mathrm{N}_{2}: \mathrm{CH}_{4}$ and $\beta-\mathrm{N}_{2}$ in the South and East pitted 1249 plains (>99\% by areal mass fraction), with no NSS endmember observed.

1250 The patchy, pitted marginal plains (unit pmp) are interpreted to represent relatively thin $\mathrm{N}_{2}$ ice 1251 (probably no more than a few hundred meters) that has condensed on rugged, low-lying uplands 1252 terrain bordering Sputnik Planitia, explaining why this unit shows a hummocky texture on a 1253 scale of several km, whereas the other plains units of Sputnik Planitia are flat. Unit pmp borders 1254 the plains of southern Sputnik Planitia, which we interpret to be a geologic consequence of the 1255 preferential deposition of volatile ices that is presently occurring within this equatorial, 1256 permanently diurnal zone (Bertrand et al., 2018), a phenomenon that is also manifested 1257 compositionally by the deeper $\mathrm{N}_{2}$ band depth in $\mathrm{N}_{2}$ ice plains at southerly latitudes (Fig. 10). As 1258 described in section 5.1, it is for this same reason that we interpret unit abm of Tenzing Montes, 1259 which is embayed by elevated $\mathrm{N}_{2}$ ice plains of unit pmp, to display a high areal mass fraction of $1260 \mathrm{~N}_{2}: \mathrm{CH}_{4}$ compared to the northern mountain ranges. The elevated $\mathrm{N}_{2}$ ice plains of unit pmp that 1261 cover the southeast margin of Sputnik Planitia (Fig. 27b) show some geomorphological 1262 similarities to the elevated plains that separate the blocks of Tenzing Montes, including scarps 1263 leading from unit pmp down to Sputnik Planitia. Unit pmp shows a similarly dense concentration 
1264 of pits to units dpp and dmp, although the pits of pmp show a smaller size range, never reaching

$1265>1 \mathrm{~km}$ in diameter. The presence of NSS endmember within unit pmp (areal mass fractions range

1266 between $20.8 \%$ and $33.2 \%$ ), however, indicates that its pits do display tholins on their floors that 1267 are detectable by LEISA.

1268 The non-Cellular plains at the eastern and southern boundaries of Sputnik Planitia are

1269 dominated by $\beta-\mathrm{N}_{2}$ ice, with typically only a few percent of $\mathrm{N}_{2}: \mathrm{CH}_{4}$. But of this $\mathrm{N}_{2}: \mathrm{CH}_{4}$, Figure

1270 8a shows that the percentage of $\mathrm{CH}_{4}$ diluted in $\mathrm{N}_{2}$ is generally high (>4\%), whereas it is low for

1271 the rest of the plains of Sputnik Planitia (generally <2\%). For the southern plains (unit dpp in

1272 South pitted plains), the elevated $\mathrm{CH}_{4}$ content may be interpreted as a manifestation of the

1273 general increase in volatile abundance that is seen towards the southern end of Sputnik Planitia,

1274 whereby the low insolation that is presently encountered here is conducive to deposition of both

$1275 \mathrm{CH}_{4}$ and $\mathrm{N}_{2}$ in low-lying areas (deposition of $\mathrm{N}_{2}$ at low elevation tends to dominate at more

1276 northerly, and more insolated, latitudes). Yet the elevated $\mathrm{CH}_{4}$ content that is seen along Sputnik

1277 Planitia's eastern boundary may instead be an indication of $\mathrm{CH}_{4}$-rich material being transported

1278 from the pitted uplands of East Tombaugh Regio by the $\mathrm{N}_{2}$ ice glaciers that lead from there into

1279 Sputnik Planitia. As noted in section 5.4, the pitted uplands likely represent a lower-elevation

1280 extension of the bladed terrain, and so their composition is likely $\mathrm{CH}_{4}$-based (Moore et al.,

1281 2018), and the scattered hills (unit sh) have accordingly been interpreted as km-sized blocks of

1282 such material that have been transported into Sputnik Planitia (albeit which are coated by

1283 freshly-deposited $\mathrm{N}_{2}$ ice). Yet given that these eastern non-Cellular plains incorporate the active

1284 glaciers leading from the uplands, their elevated $\mathrm{CH}_{4}$ content may be an indication of recently

1285 eroded, sub-resolution $\mathrm{CH}_{4}$-rich material that is being carried on the surface of the glaciers - the

1286 lateral moraine trails that have been identified at these glaciers (White et al., 2017, Howard et al., 
1287 2017) likely represent such sub-resolution material (Fig. 27c). As this material is transported

1288 farther to the west, into the interior of Sputnik Planitia, it becomes dissipated, lowering its

1289 concentration within the $\mathrm{N}_{2}$ ice, and it becomes coated by freshly deposited $\mathrm{N}_{2}$ ice (as has

1290 happened to the large scattered hills), resulting in low $\mathrm{CH}_{4}$ percentages here.

1291 While the non-Cellular plains are concentrated within southern and eastern Sputnik Planitia,

1292 units dpp and spp also form small expanses at its western and northern edges (the North and

1293 West pitted plains provinces), surrounded by the mountains and Cellular plains. Units dpp and

1294 spp within these provinces display a high albedo that is akin to the bright plains of central and

1295 southern Sputnik Planitia, and which contrasts with the darker plains of the surrounding Cellular

1296 plains (Fig. 27d). That the north and west pitted plains provinces are located within the portion of

1297 Sputnik Planitia that is presently experiencing net sublimation of $\mathrm{N}_{2}$ ice from the surface, and yet

1298 still present a high albedo, implies two possible scenarios: (i) the plains may be experiencing net

1299 sublimation like the surrounding dark Cellular plains, with the exception that the body of the $\mathrm{N}_{2}$

1300 ice forming them does not contain a high concentration of tholins that would be exposed at the

1301 surface by the sublimation, or (ii) the plains are in fact localized zones of net deposition of $\mathrm{N}_{2}$ ice

1302 rather than sublimation, causing them to be coated by a fresh, bright layer of $\mathrm{N}_{2}$ frost in the same

1303 manner as the plains in the south and east of Sputnik Planitia. These bright northern and western

1304 plains differ in composition from their southeastern counterparts: they display units dpp and spp

1305 and are composed almost entirely of $\mathrm{N}_{2}: \mathrm{CH}_{4}(>99 \%)$, in contrast to the $\beta-\mathrm{N}_{2}$ and NSS

1306 endmember that form unit dpp in the south, and the $\beta-\mathrm{N}_{2}$ and $\mathrm{N}_{2}: \mathrm{CH}_{4}$ that form unit spp in the

1307 south (Fig. 24). The most notable compositional difference is the absence of NSS endmember

1308 from unit dpp in the northern and western plains. This suggests that tholins are not covering pit

1309 floors here, in contrast to pits in unit dpp in the southern and eastern plains, and indicates that 
1310 tholins are not entrained within the $\mathrm{N}_{2}$ ice of unit dpp in the north and west. Sublimation of the

$1311 \mathrm{~N}_{2}$ ice to form the pits is not exhuming any entrained tholins, meaning that none can build up as

1312 a lag on the pit floors. If these northern and western plains are experiencing net sublimation, like

1313 the darker, cellular plains surrounding them, then the absence of entrained tholins means that the

1314 albedo of the plains will remain high regardless of how much $\mathrm{N}_{2}$ ice sublimates from the surface.

1315 In fact, the presence of localized plains that consistently display a high albedo, and which are

1316 therefore consistently cooler than the surrounding darker plains, may actually act to partially

1317 offset the predominant sublimation that occurs within this part of Sputnik Planitia, and allow

1318 them to retain more of the bright $\mathrm{N}_{2}$ ice that does deposit on them, in what would essentially be a

1319 runaway albedo effect (see point 3 in section 5.7).

1320 This does require, however, that the plains are bright to begin with, which is related to the

1321 question of how bright, non-Cellular plains were established in these marginal areas of Sputnik

1322 Planitia. They evidently represent portions of the plains at the margins of Sputnik Planitia where

1323 the $\mathrm{N}_{2}$ ice is insufficiently thick to support convection, and within which entrained tholins are

1324 absent, in contrast to the dark, adjacent Cellular plains. This observation seems to imply that

1325 convection within the Cellular plains is an efficient process for entraining tholins and ensuring

1326 that they remain trapped within the convection cells and cannot migrate to adjacent $\mathrm{N}_{2}$ ice that is

1327 not convecting. The fact that dark pit floors are seen in pitted plains of the southern non-Cellular

1328 plains suggests that glacial flow of $\mathrm{N}_{2}$ ice from the center of Sputnik Planitia to its southern

1329 regions may play a role in transferring tholin-entrained $\mathrm{N}_{2}$ ice of the convective cells outside of

1330 the convective regime. There is evidence for this in the form of elongated, "extinct" cells

1331 extending from the southern boundary of the bright Cellular plains that are defined by chains of

1332 joined, dark-floored pits that describe polygonal outlines (Fig. 27e), and which White et al. 
1333 (2017) interpreted to be formerly active convection cells that were moved southwards and their

1334 planforms distorted by lateral flow of the ice in this region of Sputnik Planitia. In the case of the

1335 bright, non-Cellular plains of the North and West pitted plains, however, it may be that

1336 convective motion in the adjacent Cellular plains acts to effectively segregate any tholins

1337 entrained within the non-Cellular plains and trap them in the convection cells, thereby

1338 brightening the non-Cellular plains and initiating the runaway albedo effect whereby sublimation

1339 of $\mathrm{N}_{2}$ ice remains dominant in the dark Cellular plains, but becomes less effective relative to

1340 deposition of $\mathrm{N}_{2}$ ice in the bright, non-Cellular plains.

13425.7 Possible processes responsible for the observed diversity of ice mixtures in Sputnik

\section{Planitia}

1344 The diversity of ice mixtures and $\mathrm{N}_{2}$ or $\mathrm{CH}_{4}$ enrichment at the surface of the plains of Sputnik

1345 Planitia may in part be a consequence of various phenomena that would not manifest in

1346 geomorphology that is resolvable by New Horizons imaging. These include: (1) Differences in

1347 ice thickness (related to different bedrock topography and glacial flow), which would impact

1348 deep ice convection and entrainment of tholins, as well as the subsurface heat flow. Within the

1349 convective cells, the subsurface heat flow would be larger at the centers than at the edges of the

1350 cells. (2) Eolian processes disrupting the surface ice (changes in albedo, composition, grain size,

1351 etc.) such as (a) warming by the atmospheric downward sensible flux, (b) mechanical erosion,

1352 and (c) atmospheric transport, surface accumulation of haze particles or dark materials and

1353 subsequent darkening of the ice (or brightening if ice grains are transported too). Bertrand et al.

1354 (2020b) investigated these processes and showed that the former and the latter could

1355 significantly impact the surface ice and the volatile sublimation-condensation rates and therefore 
1356 the surface composition. (3) Albedo and composition positive feedbacks have been shown to be 1357 very efficient in Sputnik Planitia (Earle et al., 2018, Bertrand et al., 2020b). In particular,

1358 Bertrand et al. (2020b) showed that a local decrease in $\mathrm{N}_{2}$ ice surface albedo from 0.7 to 0.65

1359 during northern spring is sufficient to turn a $\mathrm{N}_{2}$ condensation-dominated region into a

1360 sublimation-dominated region (see Fig. 16 in Bertrand et al., 2020b). The processes mentioned in

1361 (1) and (2) can locally trigger a change in surface albedo and composition that is likely to

1362 amplify with time (e.g. local deposition of $\mathrm{N}_{2}$ ice on bright surfaces and removal on dark

1363 surfaces) (4) The composition of the surface ice can also be impacted by ice segregation (e.g.

1364 formation of a $\mathrm{CH}_{4}$-rich layer on top of a $\mathrm{N}_{2}$-rich ice) and by the relaxation of the surface ice

1365 toward a ternary phase equilibrium, whose timescale and properties can vary locally depending

1366 on the local surface and atmospheric conditions (Protopapa et al., 2017, Schmitt et al., 2017, Tan

1367 and Kargel, 2019). (5) Transport of gaseous $\mathrm{CH}_{4}$ by the near-surface winds in Sputnik Planitia

1368 can also favor $\mathrm{CH}_{4}$ saturation above the surface at specific locations, and subsequent

1369 condensation and enrichment of the ice in $\mathrm{CH}_{4}$. This is illustrated by Figure 28, which shows the

1370 diurnal mean near surface $\mathrm{CH}_{4}$ atmospheric mixing ratio and the diurnal net $\mathrm{CH}_{4}$ deposition in

1371 Sputnik Planitia, as predicted for July 2015 by global climate modeling of Pluto (Bertrand et al.,

1372 2020b). The 3D simulations show that the near-surface air in Sputnik Planitia is enriched in $\mathrm{N}_{2}$

1373 (depleted in $\mathrm{CH}_{4}$ ) compared to the air outside the basin. This is due to $\mathrm{N}_{2}$ sublimation in Sputnik

1374 Planitia (and subsequent injection of cold $\mathrm{N}_{2}$-rich air in the atmosphere) and to the confinement

1375 of this N2-rich air by the high relief boundaries of the basin (Forget et al., 2017, Bertrand et al.,

1376 2020b). The near-surface northern regions of Sputnik Planitia were particularly depleted in $\mathrm{CH}_{4}$

1377 in 2015 due to the north-to-south sublimation flow that transports gaseous $\mathrm{CH}_{4}$ southward. Note

1378 that the wind patterns predicted by the model inside Sputnik Planitia become different at $\sim 1 \mathrm{~km}$ 
1379 above the surface, with a strong western boundary current and an anticlockwise wind spiral (see

1380 Bertrand et al., 2020b). Figure 28 (left) shows that higher amounts of near-surface gaseous $\mathrm{CH}_{4}$

1381 are obtained locally near the boundaries of the basin, or near the blocky mountains of the western

1382 and southern sides. This is due to downslope transport of the air which is outside the basin or

1383 above the blocky mountains and which is enriched in gaseous $\mathrm{CH}_{4}$ (due to the absence of $\mathrm{N}_{2}$

1384 sublimation or the occurrence of local sublimation of $\mathrm{CH}_{4}$-rich frosts that formed on these

1385 terrains). As a result, $\mathrm{CH}_{4}$ deposition onto the plains of Sputnik Planitia is favored in the western,

1386 eastern and southern edge of Sputnik Planitia, and in particular near the blocky mountains and

1387 near the steepest slopes of the eastern side of Sputnik Planitia (Figure 28, right), where the air is

1388 highly enriched in gaseous $\mathrm{CH}_{4}$ coming from the sublimation of the $\mathrm{CH}_{4}$-rich Bladed Terrains.

Figure 24 A-O: Pie plots summarizing the composition of the geologic units in each province, as returned by the application of

1391

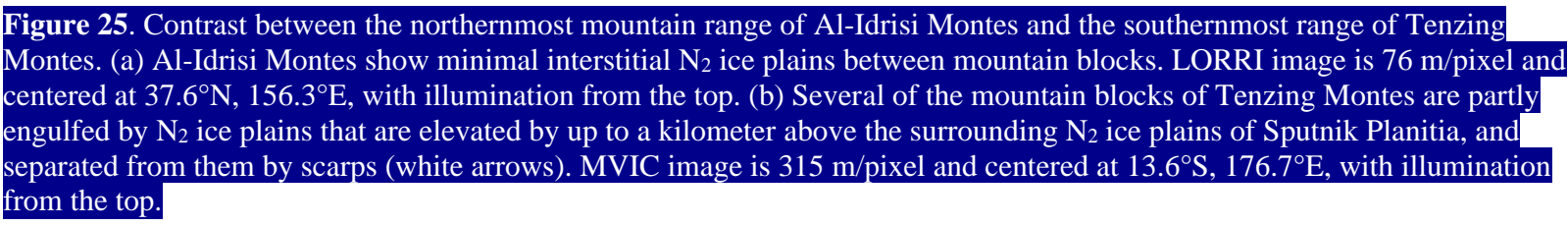




\section{Conclusions}

1433 In this study of Sputnik Planitia, we have compared spectroscopic data of this region acquired

1434 by New Horizons' LEISA instrument to the geomorphology as mapped by White et al. (2017)

1435 using visible and panchromatic imaging acquired by the LORRI and LEISA instruments.

1436 Sputnik Planitia is a unique geologic formation in our Solar System: it is a young and deep

1437 impact crater basin, hosting a large variety of diverse terrain types, who's surface veneer is made

1438 up of volatile ices captured by the cold trap created by its peculiar morphological shape. Terrain

1439 units typical of the basin's western rims, were also found inside the cellular plains, like the abm

1440 mountains, while the pitted plains covering the whole eastern edge of the planitia, are also

1441 frequent, in small patches, in most of the western territory.

1442 The idea behind this work was then to comprehend if, and in which amount, the processes

1443 responsible for the ice deposition inside the basin influence the surface composition of units

1444 belonging to the very same geological type, but that are spatially separated.

1445 We fit the units' average spectra with and Hapke theory-based modeling, and we created

1446 scatter plots comparing the integrated band depths of the main endmember's absorptions, to have

1447 a better vision of how the different surface components influence each other, and how this is

1448 reflected in the resulting surface spectra.

1449 The analysis of the $\mathrm{CH}_{4}$ integrated band depths in five wavelength ranges revealed their

1450 different sensitivities to variations in composition, grain size, and scattering properties. 
We expected to observe an anticorrelation between the $\mathrm{CH}_{4}$ absorptions and the $\mathrm{CH}_{4}$ shift

1452 index, and a positive trend between the $\mathrm{N}_{2}$ integrated band depth and the shift parameter: spectral

1453 units hosting a large amount of $\mathrm{N}_{2}$-rich ice would show high values of the shift parameter,

1454 whereas in areas where the $\mathrm{CH}_{4}$-rich ice dominates, the $\mathrm{CH}_{4}$ absorptions should manifest as

1455 small or null shifts. For three out of these six spectral indicators, the results were actually the

1456 opposite. The scatter plot of the $\mathrm{CH}_{4}$ integrated band depths versus the shift index in Ranges 1,2 ,

1457 and 4 showed, in fact, a positive correlation. We further noticed that the geologic units having

1458 weak $\mathrm{CH}_{4}$ absorptions but small values of the $\mathrm{CH}_{4}$ shift index are all located within the western

1459 mountain ranges, in addition to Columbia Colles. These units are also those that display the

1460 deepest absorptions relating to $\mathrm{H}_{2} \mathrm{O}$ and tholins, leading us to the conclusion that the weakness of

1461 the $\mathrm{CH}_{4}$ absorptions in Ranges 1, 2, and 4 for these units is not a consequence of low abundance

1462 of this material, but rather of a high concentration of other materials that mask its spectral

1463 signature or that surface particles in these units are more backscattering than those in the cellular

1464 plains. The broad $\mathrm{CH}_{4}$ absorptions in Ranges 1, 2, and 4 saturate quickly, even though not

1465 linearly, with increasing $\mathrm{CH}_{4}$ abundance, grain size, or forward scattering, while the absorption

1466 in Range 3 and the band centered at $1.48 \mu \mathrm{m}$, being weaker, require a larger quantity of $\mathrm{CH}_{4} \mathrm{for}$

1467 reaching the saturation level. This conclusion is also supported by the absence of trends between

1468 the $\mathrm{CH}_{4}$ absorptions in Ranges 1, 2, and 4 and the band in Range 3 (Figure 14).

1469 We explored the correlation between the $\mathrm{CH}_{4}$ and $\mathrm{N}_{2}$ absorptions with the other endmembers

1470 considered - $\mathrm{CO}, \mathrm{H}_{2} \mathrm{O}$, NSS endmember - to understand which one is the most responsible for

1471 the unexpected suppression of the absorptions in Ranges 1, 2, and 4 observed in Figure 13A.

1472 From the comparison between the trends in Figure 13A and Figure $16 \mathrm{~A}\left(\mathrm{CH}_{4}\right.$ integrated band

1473 depths in Ranges 1, 2, and 4 versus the CO integrated band depth), we notice that geologic units 
1474 showing deeper $\mathrm{CH}_{4}$ absorptions and higher values of the $\mathrm{CH}_{4}$ shift index also have strong $\mathrm{CO}$

1475 bands. $\mathrm{CH}_{4}$ in Range 3 and $\mathrm{N}_{2}$, on the other hand, do not show any correlation to CO (Figure

1476 16B). Grundy et al. (2013, 2014) and Schmitt et al. (2017) noticed, however, a strong correlation

1477 between $\mathrm{CO}$ and $\mathrm{N}_{2}$, but not between $\mathrm{CO}$ and $\mathrm{CH}_{4}$. Comparing the $\mathrm{CO}$ abundance map (Figure

1478 15) to the areal mass fraction maps of $\mathrm{N}_{2}: \mathrm{CH}_{4}$ (Figure 11a) and pure $\beta-\mathrm{N}_{2}$ (Figure 12a) confirms

1479 the observed connection between $\mathrm{CO}$ and $\mathrm{N}_{2}$. The fact that this link doesn't manifest in the

1480 scatter plots of Figure 16 can signify that the contaminants affect the depth of the CO absorption

1481 as well, and that $\mathrm{CO}$ is not responsible for the observed anomaly in Figure 13A.

1482 We then explored the mutual variation of the $\mathrm{CH}_{4}$ and $\mathrm{N}_{2}$ integrated band depths with the

1483 abundance of crystalline $\mathrm{H}_{2} \mathrm{O}$ and the NSS endmember returned by the modeling. The results

1484 (from Figure 18 to Figure 22) indicate that the NSS endmember (likely tholins) or more

1485 backscattering particles are primarily responsible for the suppression of $\mathrm{CH}_{4}$ absorptions in

1486 Ranges 1, 2, and 4 in the western mountainous units of Sputnik Planitia.

1487 The Hapke theory-based modeling is a powerful tool to investigate the nature of the

1488 endmembers covering a planetary surface, but it is limited by the availability and the quality of

1489 the optical constants. For instance, in our modeling we could not include the contribution of the

1490 CO because of lack of reliable optical constants, making its important contribution to the

1491 observed spectra only partially explored. The same can be concluded for the refractory non-ice

1492 material responsible for the observed negative spectral slope in the NIR spectra. Because there is

1493 very little published information on the optical constants for tholins with a composition relevant

1494 to Pluto's surface, the nature of the non-ice material is still unknown.

1495 The spectroscopic analyses of this study have provided additional insight into the geological 1496 processes that have shaped Sputnik Planitia, and in some instances they reveal aspects of the 
1497 geology that is not apparent within the visible and panchromatic imaging that was used to define 1498 the geological units in the mapping of White et al. (2017). For non-plains units, a general

1499 increase in volatile abundance from the north to the south of Sputnik Planitia is seen, manifested 1500 especially by the higher areal mass fraction of $\mathrm{N}_{2}: \mathrm{CH}_{4}$ at the expense of $\mathrm{H}_{2} \mathrm{O}$ ice for the 1501 southernmost mountains (unit abm) as compared to the northernmost ones. Higher amounts of 1502 pure $\mathrm{CH}_{4}$ are also seen at the southern mountains, which are a natural site for $\mathrm{CH}_{4}$ deposition 1503 owing to their high elevation and the lower insolation they are presently receiving; the existence 1504 of a thin veneer of pure $\mathrm{CH}_{4}$ ice is only apparent in the spectra, and not in the visible imaging. 1505 Topography within southern Sputnik Planitia (specifically the scattered hills, Tenzing Montes 1506 and Krun Macula) that appears inundated by $\mathrm{N}_{2}$ ice is interpreted as a geomorphological 1507 consequence of the increased deposition of volatiles occurring here, which is predicted by 1508 climate modeling (Bertrand et al., 2018; 2020b). The NSS endmember that has been identified 1509 in the spectra correlates to the existence of tholins within certain units, and are coating the low1510 latitude mountain ranges of Zheng He, Baret, and Hillary Montes (i.e. those that are co1511 latitudinal with the tholin-covered Cthulhu Macula in the uplands to the west) and the dark 1512 ridged terrain (unit drt); covering the floors of large pits within non-cellular plains; and are 1513 entrained within the exposed $\mathrm{N}_{2}$ ice of Sputnik Planitia within the northern dark cellular plains 1514 and trough-bounding plains (units dep and tbp). Our spectral and geomorphological 1515 observations have also revealed that Columbia Colles, which belong to the $\mathrm{CH}_{4}$ ice-based 1516 scattered hills that have been eroded from the pitted uplands of East Tombaugh Regio (unit sh), 1517 appear to have been affected by processes somewhat similar to those that have formed the dark 1518 ridged terrain along the western boundary of Sputnik Planitia, which is based on much older, $1519 \mathrm{H}_{2} \mathrm{O}$ ice-based crustal material that also forms the western mountain ranges. Elevated $\mathrm{CH}_{4}$ 
1520 concentrations within $\mathrm{N}_{2}: \mathrm{CH}_{4}$ ice of the eastern non-cellular plains may represent sub-resolution,

$1521 \mathrm{CH}_{4}$-rich material that has been recently eroded from the pitted uplands of East Tombaugh

1522 Regio, and which is being transported into Sputnik Planitia by $\mathrm{N}_{2}$ ice glaciers. The spectral

1523 analysis has also revealed compositional and/or directional scattering differences between the

1524 handful of occurrences of northern non-cellular plains and the surrounding cellular plains, all of

1525 which are located within the portion of Sputnik Planitia that is presently experiencing net

1526 sublimation of volatiles, and which do not therefore exhibit a surface layer of bright, freshly-

1527 deposited $\mathrm{N}_{2}$ ice. The compositional differences between the cellular and non-cellular plains

1528 here hint at the effectiveness of convection in entraining and trapping tholins within the body of

1529 the cellular plains, while preventing the spread of such tholins to abutting non-cellular plains.

1530 The results of this paper form a testament to the dynamism of volatile mobilization within the

1531 plains of Sputnik Planitia and its mountain ranges, with many factors including surface elevation,

1532 Pluto's highly variable climate with latitude, and endogenous factors (i.e. convection) all playing

1533 important roles in explaining our spectroscopic and geomorphological observations. As

1534 described by Bertrand et al. (2020b) Sputnik Planitia does represent the "beating heart" of Pluto

1535 that regulates its atmospheric circulation through such volatile mobilization, and the effects of

1536 this have consequences across the rest of the dwarf planet. Outside of Sputnik Planitia, both

1537 latitudinal and longitudinal variations in the distribution of surface volatiles have been described.

1538 The eastwards sequence of equatorial terrains from the bright pitted uplands of East Tombaugh

1539 Regio through the bladed terrain deposits of Tartarus Dorsa to the dark, tholin covered, cratered

1540 plains of Cthulhu Macula appear to manifest a surficial composition sequence from dominance

1541 by nitrogen directly to the east of Sputnik Planitia, to increasing dominance of methane ice

1542 farther to the east culminating in the bladed terrain deposits (Moore et al., 2018), eventually 
1543 terminating at the volatile-poor expanse of Cthulhu Macula to the west of Sputnik Planitia.

1544 Development of albedo contrasts is heightened in the equatorial region, owing to its relatively

1545 stable climate (Earle et al., 2018), and is a powerful driving force behind the very pronounced

1546 segregation of volatile-rich and -poor terrains at the equator. The drastic climate cycles

1547 experienced by the northern latitudes promotes mixing of volatiles and causes them to neither be

1548 substantially eroded nor accumulated relative to the equatorial region, but longitudinal variation

1549 is still apparent, from the smooth, mantled, undulating uplands of Hayabusa and Pioneer Terrae

1550 to the northeast of Sputnik Planitia, to the very flat, heavily cratered, partly eroded plains of

1551 Vega and Venera Terrae to the northwest. At both at low and high latitudes, the observed

1552 longitudinal variation of uplands geomorphology may indicate an origin related to the

1553 retrorotation of Pluto's atmosphere that is controlled by Sputnik Planitia, and both further high-

1554 resolution, long-term global climate model simulations and comprehensive analysis of the

1555 spectral properties of terrains beyond Sputnik Planitia will complement geological observations

1556 to expand our understanding of volatile mobilization across Pluto in general. 
1559

1560

1561

1562

1563

1564

1565

1566

1567

1568

1569

1570

1571

1572

1573

1574

1575

1576

1577

1578

1579

1580

1581

1582

1583

1584

1585

1586

1587

1588

1589

1590

1591

1592

1593

1594

1595

1596

1597

1598

1599

1600

1601

1602

Ahrens, C. J., \& Chevrier, V. F. (2019). Compressional Ridges on Baret Montes, Pluto as Observed by New Horizons. Geophysical Research Letters, 46(24), 14328-14335. https://doi.org/10.1029/2019GL085648

Bertrand, T., Forget, F., Umurhan, O. M., Grundy, W. M., Schmitt, B., Protopapa, S., Zangari, A. M., White, O. L., Schenk, P. M., Singer, K. N., Stern, A., Weaver, H. A., Young, L. A., Ennico, K., \& Olkin, C. B. (2018). The nitrogen cycles on Pluto over seasonal and astronomical timescales. Icarus, 309, 277-296. https://doi.org/10.1016/j.icarus.2018.03.012

Bertrand, T., Forget, F., Umurhan, O. M., Moore, J. M., Young, L. A., Protopapa, S., Grundy, W. M., Schmitt, B., Dhingra, R. D., Binzel, R. P., Earle, A. M., Cruikshank, D. P., Stern, S. A., Weaver, H. A., Ennico, K., \& Olkin, C. B. (2019). The CH 4 cycles on Pluto over seasonal and astronomical timescales. Icarus, 329(December 2018), 148-165. https://doi.org/10.1016/j.icarus.2019.02.007

Bertrand, Tanguy, Forget, F., Schmitt, B., White, O. L., \& Grundy, W. M. (2020a). Equatorial mountains on Pluto are covered by methane frosts resulting from a unique atmospheric process. Nature Communications, 11(1), 1-7. https://doi.org/10.1038/s41467-020-18845-3

Bertrand, T., Forget, F., White, O., Schmitt, B., Stern, S. A., Weaver, H. A., Young, L. A., Ennico, K., \& Olkin, C. B. (2020b). Pluto's Beating Heart Regulates the Atmospheric Circulation: Results From High-Resolution and Multiyear Numerical Climate Simulations. Journal of Geophysical Research: Planets, 125(2), 1-64. https://doi.org/10.1029/2019JE006120

Bertrand, Tanguy, \& Forget, F. (2016). Observed glacier and volatile distribution on Pluto from atmosphere-topography processes. Nature, 540(7631), 86-89. https://doi.org/10.1038/nature19337

Bertrand, Tanguy, \& Forget, F. (2017). 3D modeling of organic haze in Pluto's atmosphere. Icarus, 287, 72-86. https://doi.org/10.1016/j.icarus.2017.01.016

Binzel, R. P., Earle, A. M., Buie, M. W., Young, L. A., Stern, S. A., Olkin, C. B., Ennico, K., Moore, J. M., Grundy, W., Weaver, H. A., Lisse, C. M., \& Lauer, T. R. (2017). Climate zones on Pluto and Charon. Icarus, 287, 30-36. https://doi.org/10.1016/j.icarus.2016.07.023

Brunetto, R., Caniglia, G., Baratta, G. A., \& Palumbo, M. E. (2008). Integrated Near-Infrared Band Strengths of Solid CH 4 and Its Mixtures with N 2 . The Astrophysical Journal, 686(2), 1480-1485. https://doi.org/10.1086/591509

Buhler, P. B., \& Ingersoll, A. P. (2018). Sublimation pit distribution indicates convection cell surface velocities of $\sim 10 \mathrm{~cm}$ per year in Sputnik Planitia, Pluto. Icarus, 300, 327-340. https://doi.org/10.1016/j.icarus.2017.09.018

Buie, M. W., Grundy, W. M., Young, E. F., Young, L. A., \& Stern, S. A. (2010). Pluto and charon with the hubble space telescope. II. Resolving changes on pluto's surface and a map for charon. Astronomical Journal, 139(3), 1128-1143. https://doi.org/10.1088/0004$6256 / 139 / 3 / 1128$

Buratti, B. J., Hofgartner, J. D., Hicks, M. D., Weaver, H. A., Stern, S. A., Momary, T., Mosher, J. A., Beyer, R. A., Verbiscer, A. J., Zangari, A. M., Young, L. A., Lisse, C. M., Singer, K., Cheng, A., Grundy, W., Ennico, K., \& Olkin, C. B. (2017). Global albedos of Pluto and Charon from LORRI New Horizons observations. Icarus, 287, 207-217. https://doi.org/10.1016/j.icarus.2016.11.012

Cheng, A. F., Summers, M. E., Gladstone, G. R., Strobel, D. F., Young, L. A., Lavvas, P., 
1603

1604

1605

1606

1607

1608

1609

1610

1611

1612

1613

1614

1615

1616

1617

1618

1619

1620

1621

1622

1623

1624

1625

1626

1627

1628

1629

1630

1631

1632

1633

1634

1635

1636

1637

1638

1639

1640

1641

1642

1643

1644

1645

1646

1647

1648

Kammer, J. A., Lisse, C. M., Parker, A. H., Young, E. F., Stern, S. A., Weaver, H. A., Olkin, C. B., \& Ennico, K. (2017). Haze in Pluto’s atmosphere. Icarus, 290, 112-133. https://doi.org/10.1016/j.icarus.2017.02.024

Cheng, A. F., Weaver, H. A., Conard, S. J., Morgan, M. F., Barnouin-Jha, O., Boldt, J. D., Cooper, K. A., Darlington, E. H., Grey, M. P., Hayes, J. R., Kosakowski, K. E., Magee, T., Rossano, E., Sampath, D., Schlemm, C., \& Taylor, H. W. (2008). Long-range reconnaissance imager on New Horizons. Space Science Reviews, 140(1-4), 189-215. https://doi.org/10.1007/s11214-007-9271-6

Cook, J. C., Dalle Ore, C. M., Protopapa, S., Binzel, R. P., Cruikshank, D. P., Earle, A., Grundy, W. M., Ennico, K., Howett, C., Jennings, D. E., Lunsford, A. W., Olkin, C. B., Parker, A. H., Philippe, S., Reuter, D., Schmitt, B., Singer, K., Stansberry, J. A., Stern, S. A., ... Tegler, S. C. (2019). The distribution of $\mathrm{H} 2 \mathrm{O}, \mathrm{CH} 3 \mathrm{OH}$, and hydrocarbon-ices on Pluto: Analysis of New Horizons spectral images. Icarus, 331, 148-169.

https://doi.org/10.1016/j.icarus.2018.09.012

Cruikshank, D. P, Clemett, S. J., Grundy, W. M., Stern, S. A., Olkin, C. B., Binzel, R. P., Cook, J. C., Ore, C. M. D., Earle, A. M., Smith-Ennico, K., Jennings, D. E., Howett, C. J. A., Linscott, I. R., Lunsford, A. W., Parker, A. H., Parker, J. W., Protopapa, S., Reuter, D. C., Singer, K. N., ... Hiroi, T. (2016). PLUTO AND CHARON: THE NON-ICE SURFACE COMPONENT. Lunar and Planetary Science Conference XLVII, 46-47. https://doi.org/10.1038/ngeo2474.

Cruikshank, D.P., Grundy, W. M., DeMeo, F. E., Buie, M. W., Binzel, R. P., Jennings, D. E., Olkin, C. B., Parker, J. W., Reuter, D. C., Spencer, J. R., Stern, S. A., Young, L. A., \& Weaver, H. A. (2015). The surface compositions of Pluto and Charon. Icarus, 246, 82-92. https://doi.org/10.1016/j.icarus.2014.05.023

Cruikshank, D.P., Pilcher, C. B., \& Morrison, D. (1976). Pluto: Evidence for Methane Frost Abstract. Science, 194(4268), 836. https://doi.org/10.1126/science.194.4268.935

Cruikshank, Dale P., Dalle Ore, C. M., Scipioni, F., Beyer, R. A., White, O. L., Moore, J. M., Grundy, W. M., Schmitt, B., Runyon, K. D., Keane, J. T., Robbins, S. J., Stern, S. A., Bertrand, T., Beddingfield, C. B., Olkin, C. B., Young, L. A., Weaver, H. A., \& Ennico, K. (2020). Cryovolcanic flooding in Viking Terra on Pluto. Icarus, November 2019, 113786. https://doi.org/10.1016/j.icarus.2020.113786

Cruikshank, Dale P., Hamilton Brown, R., \& Clark, R. N. (1984). Nitrogen on Triton. Icarus, 58(2), 293-305. https://doi.org/10.1016/0019-1035(84)90046-0

Douté, S., Schmitt, B., Quirico, E., Owen, T. C., Cruikshank, D. P., De Bergh, C., Geballe, T. R., \& Roush, T. L. (1999). Evidence for Methane Segregation at the Surface of Pluto. Icarus, 142(2), 421-444. https://doi.org/10.1006/icar.1999.6226

Earle, A. M., Binzel, R. P., Young, L. A., Stern, S. A., Ennico, K., Grundy, W., Olkin, C. B., \& Weaver, H. A. (2017). Long-term surface temperature modeling of Pluto. Icarus, 287, 3746. https://doi.org/10.1016/j.icarus.2016.09.036

Earle, A. M., Binzel, R. P., Young, L. A., Stern, S. A., Ennico, K., Grundy, W., Olkin, C. B., \& Weaver, H. A. (2018). Albedo matters: Understanding runaway albedo variations on Pluto. Icarus, 303, 1-9. https://doi.org/10.1016/j.icarus.2017.12.015

Gladstone, G. R., Stern, S. A., Ennico, K., Olkin, C. B., Weaver, H. A., Young, L. A., Summers, M. E., Strobel, D. F., Hinson, D. P., Kammer, J. A., Parker, A. H., Steffl, A. J., Linscott, I. R., Parker, J. W., Cheng, A. F., Slater, D. C., Versteeg, M. H., Greathouse, T. K., Retherford, K. D., ... Tyler, G. L. (2016). The atmosphere of Pluto as observed by New 
1662

1663

1664

1665

1666

1667

1668

1669

1670

1671

1672

1673

1674

1675

1676

1677

1678

1679

1680

1681

1682

1683

1684

1685

1686

1687

1688

1689

1690

1691

1692

1693

1694

Horizons. Science, 351(6279). https://doi.org/10.1126/science.aad8866

Grundy, W. M., Bertrand, T., Binzel, R. P., Buie, M. W., Buratti, B. J., Cheng, A. F., Cook, J. C., Cruikshank, D. P., Devins, S. L., Dalle Ore, C. M., Earle, A. M., Ennico, K., Forget, F., Gao, P., Gladstone, G. R., Howett, C. J. A., Jennings, D. E., Kammer, J. A., Lauer, T. R., ... Zhang, X. (2018). Pluto's haze as a surface material. Icarus, 314(June), 232-245. https://doi.org/10.1016/j.icarus.2018.05.019

Grundy, W. M., Binzel, R. P., Buratti, B. J., Cook, J. C., Cruikshank, D. P., Dalle Ore, C. M., Earle, A. M., Ennico, K., Howett, C. J. A., Lunsford, A. W., Olkin, C. B., Parker, A. H., Philippe, S., Protopapa, S., Quirico, E., Reuter, D. C., Schmitt, B., Singer, K. N., Verbiscer, A. J., ... Young, L. A. (2016). Surface compositions across Pluto and Charon. Science, 351(6279). https://doi.org/10.1126/science.aad9189

Grundy, W. M. I., \& Schmitt, B. (1998). The temperature-dependent near-infrared absorption spectrum of hexagonal ice. Journal of Geophysical Research, 103, 809-822.

Grundy, W. M., Olkin, C. B., Young, L. A., Buie, M. W., \& Young, E. F. (2013). Near-infrared spectral monitoring of Pluto's ices: Spatial distribution and secular evolution. Icarus, 223(2), 710-721. https://doi.org/10.1016/j.icarus.2013.01.019

Grundy, W. M., Olkin, C. B., Young, L. A., \& Holler, B. J. (2014). Near-infrared spectral monitoring of Pluto's ices II: Recent decline of CO and N2 ice absorptions. Icarus, 235, 220-224. https://doi.org/10.1016/j.icarus.2014.02.025

Grundy, W. M., Schmitt, B., \& Quirico, E. (1993). The Temperature-Dependent Spectra of $\alpha$ and $\beta$ Nitrogen Ice with Application to Triton. In Icarus (Vol. 105, Issue 1, pp. 254-258). https://doi.org/10.1006/icar.1993.1122

Grundy, W. M., Schmitt, B., \& Quirico, E. (2002). The temperature-dependent spectrum of methane ice I between 0.7 and $5 \mu \mathrm{m}$ and opportunities for near-infrared remote thermometry. Icarus, 155(2), 486-496. https://doi.org/10.1006/icar.2001.6726

Hamilton, D. P., Stern, S. A., Moore, J. M., \& Young, L. A. (2016). The rapid formation of Sputnik Planitia early in Pluto's history. Nature, 540(7631), 97-99. https://doi.org/10.1038/nature20586

Hapke, B. W. (2012). THEORY OF REFLECTANCE AND EMITTANCE SPECTROSCOPY (B. Hapke (ed.); 2nd ed.). Cambridge University Press.

Hapke, B. W., Nelson, R. M., \& Smythe, W. D. (1993). The Opposition Effect of the Moon : The Contribution of Coherent Backscatter. Science, 23(5107), 509-511. https://doi.org/10.1126/science.260.5107.509

Howard, A. D., Moore, J. M., Umurhan, O. M., White, O. L., Anderson, R. S., McKinnon, W. B., Spencer, J. R., Schenk, P. M., Beyer, R. A., Stern, S. A., Ennico, K., Olkin, C. B., Weaver, H. A., \& Young, L. A. (2017). Present and past glaciation on Pluto. Icarus, 287, 287-300. https://doi.org/10.1016/j.icarus.2016.07.006

Johnson, B. C., Bowling, T. J., Trowbridge, A. J., \& Freed, A. M. (2016). Formation of the Sputnik Planum basin and the thickness of Pluto's subsurface ocean. Geophysical Research Letters, 43(19), 10,068-10,077. https://doi.org/10.1002/2016GL070694

Johnson, P. E., Young, L. A., Protopapa, S., Schmitt, B., Gabasova, L. R., Lewis, B. L., Stansberry, J. A., Mandt, K. E., \& White, O. L. (2020). Modeling Pluto's minimum pressure: Implications for haze production. Icarus, August 2019. https://doi.org/10.1016/j.icarus.2020.114070

Keane, J. T., Matsuyama, I., Kamata, S., \& Steckloff, J. K. (2016). Reorientation and faulting of Pluto due to volatile loading within Sputnik Planitia. Nature, 540(7631), 90-93. 
https://doi.org/10.1038/nature20120

Khare, B. N., Sagan, C., Arakawa, E. T., Suits, F., Callcott, T. A., \& Williams, M. W. (1984). Optical constants of organic tholins produced in a simulated Titanian atmosphere: From soft x-ray to microwave frequencies. Icarus, 60(1), 127-137. https://doi.org/10.1016/00191035(84)90142-8

Khare, B. N., Sagan, C., Heinrich, M., Thompson, W. R., Arakawa, E. T., Tuminello, P. S., \& Clark, M. (1994). Optical Constants of Triton Tholin: Preliminary Results. American Astronomical Society, DPS Meeting, 26, 1176.

Khare, B. N., Thompson, W. R., Cheng, L., Chyba, C., Sagan, C., Arakawa, E. T., Meisse, C., \& Tuminello, P. S. (1993). Production and Optical Constants of Ice Tholin from Charged Particle Irradiation of (1:6) C2H6/H2O at 77 K. In Icarus (Vol. 103, Issue 2, pp. 290-300). https://doi.org/10.1006/icar.1993.1071

Lorenzi, V., Pinilla-Alonso, N., Licandro, J., Cruikshank, D. P., Grundy, W. M., Binzel, R. P., \& Emery, J. P. (2016). The spectrum of Pluto, 0.40-0.93 $\mu \mathrm{m}$ : I. Secular and longitudinal distribution of ices and complex organics. Astronomy and Astrophysics, 585, 1-12. https://doi.org/10.1051/0004-6361/201527281

Lunine, J. I., \& Stevenson, D. J. (1985). Physics and Chemistry of sulfur lakes on Io. Icarus, 64(3), 345-367. https://doi.org/10.1016/0019-1035(85)90060-0

Mastrapa, R. M., Bernstein, M. P., Sandford, S. A., Roush, T. L., Cruikshank, D. P., \& Ore, C. M. D. (2008). Optical constants of amorphous and crystalline H2O-ice in the near infrared from 1.1 to $2.6 \mu \mathrm{m}$. Icarus, 197(1), 307-320. https://doi.org/10.1016/j.icarus.2008.04.008

Materese, C. K., Cruikshank, D. P., Sandford, S. A., Imanaka, H., \& Nuevo, M. (2015). ICE CHEMISTRY on OUTER SOLAR SYSTEM BODIES: ELECTRON RADIOLYSIS of N2-, CH4-, and CO-CONTAINING ICES. Astrophysical Journal, 812(2), 150. https://doi.org/10.1088/0004-637X/812/2/150

Materese, C. K., Cruikshank, D. P., Sandford, S. A., Imanaka, H., Nuevo, M., \& White, D. W. (2014). ICE chemistry on outer solar system bodies: Carboxylic acids, NITRILES, and urea detected in refractory residues produced from the UV photolysis of $\mathrm{N} \mathrm{2:CH4:Co-containing}$ ices. Astrophysical Journal, 788(2). https://doi.org/10.1088/0004-637X/788/2/111

McKinnon, W. B., Nimmo, F., Wong, T., Schenk, P. M., White, O. L., Roberts, J. H., Moore, J. M., Spencer, J. R., Howard, A. D., Umurhan, O. M., Stern, S. A., Weaver, H. A., Olkin, C. B., Young, L. A., \& Smith, K. E. (2016). Convection in a volatile nitrogen-ice-rich layer drives Pluto's geological vigour. Nature, 534(7605), 82-85. https://doi.org/10.1038/nature18289

Merlin, F. (2015). New constraints on the surface of Pluto. Astronomy and Astrophysics, 582, 19. https://doi.org/10.1051/0004-6361/201526721

Moore, J.M., McKinnon, W. B., Spencer, J. R., Howard, A. D., Schenk, P. M., Beyer, R. A., Nimmo, F., Singer, K. N., Umurhan, O. M., White, O. L., Stern, S. A., Ennico, K., Olkin, C. B., Weaver, H. A., Young, L. A., Binzel, R. P., \& Wilhelms, D. E. (2016). The geology of Pluto and Charon through the eyes of New Horizons. Science, 351(6279).

Moore, Jeffrey M., Howard, A. D., Umurhan, O. M., White, O. L., Schenk, P. M., Beyer, R. A., McKinnon, W. B., Spencer, J. R., Singer, K. N., Grundy, W. M., Earle, A. M., Schmitt, B., Protopapa, S., Nimmo, F., Cruikshank, D. P., Hinson, D. P., Young, L. A., Stern, S. A., Weaver, H. A., ... Scipioni, F. (2018). Bladed Terrain on Pluto: Possible origins and evolution. Icarus, 300, 129-144. https://doi.org/10.1016/j.icarus.2017.08.031

Nimmo, F., Hamilton, D. P., McKinnon, W. B., Schenk, P. M., Binzel, R. P., Bierson, C. J., 
Beyer, R. A., Moore, J. M., Stern, S. A., Weaver, H. A., Olkin, C. B., Young, L. A., \& Smith, K. E. (2016). Reorientation of Sputnik Planitia implies a subsurface ocean on Pluto. Nature, 540(7631), 94-96. https://doi.org/10.1038/nature20148

O’Hara, S. T., \& Dombard, A. J. (2020). Downhill sledding at 40 AU: Mobilizing Pluto's chaotic mountain blocks. Icarus, 113829. https://doi.org/10.1016/j.icarus.2020.113829

Olkin, C. B.; Young, E. F.; Young, L. A.; Grundy, W.; Schmitt, B.; Tokunaga, A.; Owen, T.; Roush, T.; Terada, H. (2007). Pluto's Spectrum from 1.0 to $4.2 \mu \mathrm{m}$ : Implications for Surface Properties. The Astronomical Journal, Volume 133, Issue 2, pp. 420-431.

Owen, T. C., Roush, T. L., Cruikshank, D. P., Elliot, J. L., Young, L. A., de Bergh, C., Schmitt, B., Geballe, T. R., Brown, R. H., \& Bartholomew, M. J. (1993). Surface Ices and the Atmospheric Composition of Pluto. Science, 261(5122), 745-748. https://doi.org/10.1126/science.261.5122.745

Prokhvatilov, A. I., \& Yantsevich, L. D. (1983). X-ray investigation of the equilibrium phase diagram of CH4-N2 solid mixtures. Sov. J. Low Temp. Phys., 9, 94-98.

Protopapa, S. et al. (2021). Surface Composition of Charon. In: Stern, S.A., Young, L. A., Moore, J.M., Grundy, W.M., Binzel, R.P., (Eds.), The Pluto System. University of Arizona Press, Tucson.

Protopapa, S., et al. (2020). Disk-resolved Photometric Properties of Pluto and the Coloring Materials across its Surface. The Astronomical Journal, 159, 74

Protopapa, S., Grundy, W. M., Reuter, D. C., Hamilton, D. P., Dalle Ore, C. M., Cook, J. C., Cruikshank, D. P., Schmitt, B., Philippe, S., Quirico, E., Binzel, R. P., Earle, A. M., Ennico, K., Howett, C. J. A., Lunsford, A. W., Olkin, C. B., Parker, A., Singer, K. N., Stern, A., ... Young, L. A. (2017). Pluto's global surface composition through pixel-by-pixel Hapke modeling of New Horizons Ralph/LEISA data. Icarus, 287, 218-228. https://doi.org/10.1016/j.icarus.2016.11.028

Protopapa, S., Grundy, W. M., Tegler, S. C., \& Bergonio, J. M. (2015). Absorption coefficients of the methane-nitrogen binary ice system: Implications for Pluto. Icarus, 253, 179-188. https://doi.org/10.1016/j.icarus.2015.02.027

Protopapa, S. et al. (2008) Surface characterization of Pluto and Charon by L and M band spectra. $A \& A, 490,365-375$.

Quirico, E., \& Schmitt, B. (1997). A spectroscopic study of CO diluted in N2 ice: Applications for Triton and Pluto. Icarus, 128(1), 181-188. https://doi.org/10.1006/icar.1997.5710

Reuter, D. C., Stern, S. A., Scherrer, J., Jennings, D. E., Baer, J. W., Hanley, J., Hardaway, L., Lunsford, A., McMuldroch, S., Moore, J. M., Olkin, C. B., Parizek, R., Reitsma, H., Sabatke, D., Spencer, J., Stone, J., Throop, H. B., Van Cleve, J., Weigle, G. E., \& Young, L. A. (2009). Ralph: A visible/infrared imager for the new horizons pluto/kuiper belt mission. New Horizons: Reconnaissance of the Pluto-Charon System and the Kuiper Belt, Mvic, 129-154. https://doi.org/10.1007/978-0-387-89518-5_7

Robbins, S. J., Singer, K. N., Bray, V. J., Schenk, P., Lauer, T. R., Weaver, H. A., Runyon, K., McKinnon, W. B., Beyer, R. A., Porter, S., White, O. L., Hofgartner, J. D., Zangari, A. M., Moore, J. M., Young, L. A., Spencer, J. R., Binzel, R. P., Buie, M. W., Buratti, B. J., ... Stern, S. A. (2017). Craters of the Pluto-Charon system. Icarus, 287(July 2015), 187-206. https://doi.org/10.1016/j.icarus.2016.09.027

Schenk, P. M., Beyer, R. A., McKinnon, W. B., Moore, J. M., Spencer, J. R., White, O. L., Singer, K., Nimmo, F., Thomason, C., Lauer, T. R., Robbins, S. J., Umurhan, O. M., Grundy, W. M., Stern, S. A., Weaver, H. A., Young, L. A., Ennico Smith, K., \& Olkin, C. 
(2018). Basins, fractures and volcanoes: Global cartography and topography of Pluto from New Horizons. Icarus, 314(June), 400-433. https://doi.org/10.1016/j.icarus.2018.06.008 Schmitt, B., Philippe, S., Grundy, W. M., Reuter, D. C., Côte, R., Quirico, E., Protopapa, S., Young, L. A., Binzel, R. P., Cook, J. C., Cruikshank, D. P., Dalle Ore, C. M., Earle, A. M., Ennico, K., Howett, C. J. A., Jennings, D. E., Linscott, I. R., Lunsford, A. W., Olkin, C. B., ... Weaver, H. A. (2017). Physical state and distribution of materials at the surface of Pluto from New Horizons LEISA imaging spectrometer. Icarus, 287, 229-260. https://doi.org/10.1016/j.icarus.2016.12.025

Schmitt, B., \& Quirico, E. (1992). Laboratory Data on Near-Infrared Spectra of Ices of Planetary Interest. American Astronomical Society, 24th DPS Meeting.

Singer, K. N., McKinnon, W. B., Gladman, B., Greenstreet, S., Bierhaus, E. B., Stern, S. A., Parker, A. H., Robbins, S. J., Schenk, P. M., Grundy, W. M., Bray, V. J., Beyer, R. A., Binzel, R. P., Weaver, H. A., Young, L. A., Spencer, J. R., Kavelaars, J. J., Moore, J. M., Zangari, A. M., ... Ennico, K. (2019). Impact craters on Pluto and Charon indicate a deficit of small Kuiper belt objects. Science, 363(6430), 955-959. https://doi.org/10.1126/science.aap8628

Skjetne, H. L., Singer, K. N., Hynek, B. M., Knight, K. I., Schenk, P. M., Olkin, C. B., White, O. L., Bertrand, T., Runyon, K. D., McKinnon, W. B., Moore, J. M., Stern, S. A., Weaver, H. A., Young, L. A., \& Ennico, K. (2020). Morphological comparison of blocks in chaos terrains on Pluto, Europa, and Mars. Icarus, 113866. https://doi.org/10.1016/j.icarus.2020.113866

Stephan, K., Jaumann, R., Krohn, K., Schmedemann, N., Zambon, F., Tosi, F., Carrozzo, F. G., McFadden, L. A., Otto, K., De Sanctis, M. C., Ammannito, E., Matz, K. D., Roatsch, T., Preusker, F., Raymond, C. A., \& Russell, C. T. (2017). An investigation of the bluish material on Ceres. Geophysical Research Letters, 44(4), 1660-1668. https://doi.org/10.1002/2016GL071652

Stern, S. A., Bagenal, F., Ennico, K., Gladstone, G. R., Grundy, W. M., McKinnon, W. B., Moore, J. M., Olkin, C. B., Spencer, J. R., Weaver, H. A., Young, L. A., Andert, T., Andrews, J., Banks, M., Bauer, B., Bauman, J., Barnouin, O. S., Bedini, P., Beisser, K., ... Zirnstein, E. (2015). The pluto system: Initial results from its exploration by New Horizons. Science, 350(6258). https://doi.org/10.1126/science.aad1815

Stern, S. A., Binzel, R. P., Earle, A. M., Singer, K. N., Young, L. A., Weaver, H. A., Olkin, C. B., Ennico, K., Moore, J. M., McKinnon, W. B., \& Spencer, J. R. (2017). Past epochs of significantly higher pressure atmospheres on Pluto. Icarus, 287, 47-53. https://doi.org/10.1016/j.icarus.2016.11.022

Trowbridge, A. J., Melosh, H. J., Steckloff, J. K., \& Freed, A. M. (2016). Vigorous convection as the explanation for Pluto's polygonal terrain. Nature, 534(7605), 79-81. https://doi.org/10.1038/nature18016

Verbiscer, A. J., Peterson, D. E., Skrutskie, M. F., Cushing, M., Helfenstein, P., Nelson, M. J., Smith, J. D., \& Wilson, J. C. (2006). Near-infrared spectra of the leading and trailing hemispheres of Enceladus. Icarus, 182(1), 211-223. https://doi.org/10.1016/j.icarus.2005.12.008

Verbiscer, A., Showalter, M., Helfenstein, P., \& Buie, M. (2019). The Pluto System at True Opposition. EPSC-DPS Joint Meeting, 13.

Wei, Q., Hu, Y., Liu, Y., Lin, D. N. C., Yang, J., \& Showman, A. P. (2018). Young Surface of Pluto's Sputnik Planitia Caused by Viscous Relaxation. The Astrophysical Journal, 856(1), 
1833

1834

1835

1836

1837

1838

1839

1840

1841

1842

1843

1844

1845

1846

1847

1848

1849

1850

1851

1852

1853

1854

1855

1856

1857

1858

1859

1860

1861

1862

1863

1864

1865

1866

1867

1868

1869

1870

1871

1872

1873

1874

1875

1876
L14. https://doi.org/10.3847/2041-8213/aab54f

White, O. L., Moore, J. M., McKinnon, W. B., Spencer, J. R., Howard, A. D., Schenk, P. M., Beyer, R. A., Nimmo, F., Singer, K. N., Umurhan, O. M., Stern, S. A., Ennico, K., Olkin, C. B., Weaver, H. A., Young, L. A., Cheng, A. F., Bertrand, T., Binzel, R. P., Earle, A. M., ... Schmitt, B. (2017). Geological mapping of Sputnik Planitia on Pluto. Icarus, 287, 261-286. https://doi.org/10.1016/j.icarus.2017.01.011

Young, L. A., Kammer, J. A., Steffl, A. J., Gladstone, G. R., Summers, M. E., Strobel, D. F., Hinson, D. P., Stern, S. A., Weaver, H. A., Olkin, C. B., Ennico, K., McComas, D. J., Cheng, A. F., Gao, P., Lavvas, P., Linscott, I. R., Wong, M. L., Yung, Y. L., Cunningham, N., ... Versteeg, M. (2018). Structure and composition of Pluto's atmosphere from the New Horizons solar ultraviolet occultation. Icarus, 300, 174-199.

https://doi.org/10.1016/j.icarus.2017.09.006 


\section{Appendix}

1888

Table: $\mathrm{CH}_{4}$ shift parameter and integrated band depths calculated for the average spectra of the 48 provinces of Sputnik Planitia

\begin{tabular}{|c|c|c|c|c|c|c|c|c|}
\hline \multirow{3}{*}{ Province* } & \multirow[b]{2}{*}{$\begin{array}{l}\mathrm{CH}_{4} \text { shift } \\
\text { parameter }\end{array}$} & \multicolumn{7}{|c|}{ Integrated band depths } \\
\hline & & $\begin{array}{c}\mathrm{CH}_{4} \\
\text { Range 1 } \\
1.30-1.43\end{array}$ & $\begin{array}{c}\mathrm{CH}_{4} \\
\text { Range 2 } \\
1.59-1.83\end{array}$ & $\begin{array}{c}\mathrm{CH}_{4} \\
\text { Range } 3 \\
1.90-2.00\end{array}$ & $\begin{array}{c}\mathrm{CH}_{4} \\
\text { Range } 4 \\
2.09-2.48\end{array}$ & $\begin{array}{c}\mathrm{CH}_{4} \\
1.48 \mu \mathrm{m}\end{array}$ & $\begin{array}{c}\mathrm{N}_{2} \\
2.15 \mu \mathrm{m}\end{array}$ & $\begin{array}{c}\mathrm{CO} \\
1.58 \mu \mathrm{m}\end{array}$ \\
\hline & & $\mu \mathrm{m}$ & $\mu \mathrm{m}$ & $\mu \mathrm{m}$ & $\mu \mathrm{m}$ & & & \\
\hline
\end{tabular}

ABM

\begin{tabular}{|c|c|c|c|c|c|c|c|c|}
\hline $\begin{array}{c}\text { Baret } \\
\text { Montes }\end{array}$ & 0.003 & 0.08 & 0.07 & 0.05 & 0.12 & 0.02 & 0.02 & 0.009 \\
\hline $\begin{array}{c}\text { Cellular } \\
\text { plains }\end{array}$ & 0.004 & 0.29 & 0.36 & 0.06 & 0.58 & 0.04 & 0.03 & 0.04 \\
\hline $\begin{array}{l}\text { Hillary } \\
\text { Montes }\end{array}$ & 0.001 & 0.17 & 0.18 & 0.08 & 0.31 & 0.03 & 0.01 & 0.003 \\
\hline $\begin{array}{c}\text { Southwest } \\
\text { buffer }\end{array}$ & 0.003 & 0.29 & 0.32 & 0.11 & 0.50 & 0.04 & 0.02 & 0.04 \\
\hline $\begin{array}{l}\text { Tenzing } \\
\text { Montes }\end{array}$ & 0.002 & 0.23 & 0.27 & 0.10 & 0.45 & 0.04 & 0.01 & 0.02 \\
\hline $\begin{array}{l}\text { Zheng He } \\
\text { Montes }\end{array}$ & 0.001 & 0.19 & 0.21 & 0.07 & 0.36 & 0.02 & 0.01 & 0.00 \\
\hline $\begin{array}{c}\text { Al-Idrisi } \\
\text { Montes } \\
\end{array}$ & 0.005 & 0.15 & 0.23 & 0.04 & 0.42 & 0.17 & 0.16 & 0.009 \\
\hline \multicolumn{9}{|c|}{ BCP } \\
\hline $\begin{array}{c}\text { Cellular } \\
\text { plains }\end{array}$ & 0.006 & 0.29 & 0.41 & 0.05 & 0.62 & 0.04 & 0.06 & 0.03 \\
\hline \multicolumn{9}{|c|}{ BCT } \\
\hline $\begin{array}{l}\text { Al-Idrisi } \\
\text { Montes }\end{array}$ & 0.007 & 0.08 & 0.18 & 0.02 & 0.46 & 0.004 & 0.08 & 0.002 \\
\hline \multicolumn{9}{|c|}{ DCP } \\
\hline $\begin{array}{l}\text { Cellular } \\
\text { plains }\end{array}$ & 0.005 & 0.27 & 0.36 & 0.05 & 0.58 & 0.04 & 0.04 & 0.02 \\
\hline $\begin{array}{l}\text { Al-Idrisi } \\
\text { Montes }\end{array}$ & 0.007 & 0.14 & 0.26 & 0.02 & 0.48 & 0.03 & 0.06 & 0.01 \\
\hline \multicolumn{9}{|c|}{ DMP } \\
\hline Baret & 0.002 & 0.29 & 0.34 & 0.07 & 0.52 & 0.05 & 0.03 & 0.01 \\
\hline
\end{tabular}




\begin{tabular}{|c|c|c|c|c|c|c|c|c|}
\hline Montes & & & & & & & & \\
\hline $\begin{array}{l}\text { Hillary } \\
\text { Montes }\end{array}$ & 0.003 & 0.29 & 0.34 & 0.08 & 0.52 & 0.04 & 0.03 & 0.02 \\
\hline \multicolumn{9}{|c|}{ DPP } \\
\hline $\begin{array}{l}\text { Hillary } \\
\text { Montes }\end{array}$ & 0.002 & 0.27 & 0.31 & 0.09 & 0.49 & 0.05 & 0.04 & 0.01 \\
\hline $\begin{array}{l}\text { North } \\
\text { pitted } \\
\text { plains }\end{array}$ & 0.005 & 0.25 & 0.36 & 0.05 & 0.58 & 0.04 & 0.06 & 0.02 \\
\hline $\begin{array}{c}\text { Cellular } \\
\text { plains }\end{array}$ & 0.004 & 0.30 & 0.38 & 0.06 & 0.58 & 0.05 & 0.04 & 0.02 \\
\hline $\begin{array}{l}\text { East pitted } \\
\text { plains }\end{array}$ & 0.005 & 0.29 & 0.40 & 0.06 & 0.59 & 0.05 & 0.04 & 0.03 \\
\hline $\begin{array}{l}\text { South } \\
\text { pitted } \\
\text { plains }\end{array}$ & 0.004 & 0.30 & 0.39 & 0.07 & 0.60 & 0.05 & 0.06 & 0.02 \\
\hline $\begin{array}{c}\text { West pitted } \\
\text { plains } \\
\end{array}$ & 0.003 & 0.30 & 0.36 & 0.06 & 0.57 & 0.04 & 0.06 & 0.02 \\
\hline \multicolumn{9}{|c|}{ DRT } \\
\hline $\begin{array}{c}\text { Baret } \\
\text { Montes }\end{array}$ & 0.002 & 0.06 & 0.05 & 0.04 & 0.09 & 0.008 & 0.01 & 0.00 \\
\hline $\begin{array}{l}\text { Hillary } \\
\text { Montes }\end{array}$ & 0.004 & 0.15 & 0.16 & 0.09 & 0.27 & 0.02 & 0.02 & 0.00 \\
\hline $\begin{array}{l}\text { Al-Idrisi } \\
\text { Montes }\end{array}$ & 0.003 & 0.06 & 0.05 & 0.05 & 0.12 & 0.002 & 0.02 & 0.009 \\
\hline \multicolumn{9}{|c|}{ IBM } \\
\hline $\begin{array}{c}\text { Baret } \\
\text { Montes }\end{array}$ & 0.003 & 0.16 & 0.18 & 0.07 & 0.33 & 0.04 & 0.02 & 0.01 \\
\hline $\begin{array}{l}\text { Hillary } \\
\text { Montes }\end{array}$ & 0.002 & 0.24 & 0.28 & 0.08 & 0.43 & 0.04 & 0.04 & 0.01 \\
\hline $\begin{array}{l}\text { Tenzing } \\
\text { Montes }\end{array}$ & 0.003 & 0.25 & 0.32 & 0.10 & 0.51 & 0.04 & 0.03 & 0.03 \\
\hline $\begin{array}{l}\text { Al-Idrisi } \\
\text { Montes }\end{array}$ & 0.005 & 0.11 & 0.20 & 0.04 & 0.40 & 0.01 & 0.04 & 0.008 \\
\hline \multicolumn{9}{|c|}{ LPP } \\
\hline $\begin{array}{l}\text { Hillary } \\
\text { Montes }\end{array}$ & 0.003 & 0.31 & 0.35 & 0.07 & 0.57 & 0.04 & 0.06 & 0.00 \\
\hline $\begin{array}{l}\text { East pitted } \\
\text { plains }\end{array}$ & 0.005 & 0.30 & 0.41 & 0.06 & 0.62 & 0.04 & 0.06 & 0.03 \\
\hline $\begin{array}{c}\text { South pitted } \\
\text { plains }\end{array}$ & 0.006 & 0.27 & 0.40 & 0.04 & 0.62 & 0.03 & 0.08 & 0.03 \\
\hline $\begin{array}{c}\text { Cellular } \\
\text { plains }\end{array}$ & 0.005 & 0.29 & 0.39 & 0.04 & 0.60 & 0.04 & 0.05 & 0.04 \\
\hline $\begin{array}{c}\text { Al-Idrisi } \\
\text { Montes } \\
\end{array}$ & 0.005 & 0.21 & 0.30 & 0.06 & 0.49 & 0.03 & 0.02 & 0.004 \\
\hline \multicolumn{9}{|c|}{ PMP } \\
\hline $\begin{array}{c}\text { East pitted } \\
\text { plains }\end{array}$ & 0.004 & 0.30 & 0.40 & 0.07 & 0.59 & 0.05 & 0.04 & 0.02 \\
\hline $\begin{array}{c}\text { Southwest } \\
\text { buffer }\end{array}$ & 0.003 & 0.31 & 0.36 & 0.10 & 0.55 & 0.05 & 0.02 & 0.02 \\
\hline
\end{tabular}




\begin{tabular}{|c|c|c|c|c|c|c|c|c|}
\hline $\begin{array}{l}\text { South } \\
\text { pitted } \\
\text { plains }\end{array}$ & 0.004 & 0.29 & 0.39 & 0.07 & 0.58 & 0.05 & 0.03 & 0.02 \\
\hline $\begin{array}{l}\text { Tenzing } \\
\text { Montes }\end{array}$ & 0.003 & 0.25 & 0.36 & 0.07 & 0.57 & 0.03 & 0.02 & 0.03 \\
\hline \multicolumn{9}{|c|}{ SH } \\
\hline $\begin{array}{l}\text { Astrid } \\
\text { Colles }\end{array}$ & 0.005 & 0.27 & 0.39 & 0.05 & 0.59 & 0.05 & 0.04 & 0.03 \\
\hline $\begin{array}{c}\text { Challenger } \\
\text { Colles }\end{array}$ & 0.003 & 0.26 & 0.34 & 0.06 & 0.52 & 0.03 & 0.04 & 0.02 \\
\hline $\begin{array}{c}\text { Columbia } \\
\text { Colles }\end{array}$ & 0.003 & 0.18 & 0.23 & 0.04 & 0.40 & 0.03 & 0.04 & 0.01 \\
\hline $\begin{array}{l}\text { Soyuz } \\
\text { Colles } \\
\end{array}$ & 0.005 & 0.33 & 0.40 & 0.06 & 0.60 & 0.04 & 0.008 & 0.03 \\
\hline $\begin{array}{c}\text { East pitted } \\
\text { plains } \\
\end{array}$ & 0.005 & 0.30 & 0.40 & 0.07 & 0.61 & 0.03 & 0.04 & 0.02 \\
\hline \multicolumn{9}{|c|}{ SPP } \\
\hline $\begin{array}{l}\text { Hillary } \\
\text { Montes }\end{array}$ & 0.003 & 0.33 & 0.39 & 0.08 & 0.59 & 0.04 & 0.05 & 0.01 \\
\hline $\begin{array}{l}\text { East pitted } \\
\text { plains }\end{array}$ & 0.005 & 0.30 & 0.38 & 0.06 & 0.60 & 0.02 & 0.05 & 0.03 \\
\hline $\begin{array}{c}\text { South pitted } \\
\text { plains }\end{array}$ & 0.005 & 0.29 & 0.40 & 0.05 & 0.61 & 0.04 & 0.08 & 0.03 \\
\hline $\begin{array}{l}\text { North } \\
\text { pitted } \\
\text { plains }\end{array}$ & 0.005 & 0.27 & 0.35 & 0.03 & 0.59 & 0.04 & 0.07 & 0.01 \\
\hline $\begin{array}{l}\text { Cellular } \\
\text { plains }\end{array}$ & 0.005 & 0.29 & 0.38 & 0.03 & 0.60 & 0.04 & 0.05 & 0.03 \\
\hline $\begin{array}{l}\text { Southwest } \\
\text { buffer }\end{array}$ & 0.003 & 0.33 & 0.41 & 0.08 & 0.62 & 0.05 & 0.06 & 0.03 \\
\hline $\begin{array}{l}\text { West pitted } \\
\text { plains } \\
\end{array}$ & 0.003 & 0.30 & 0.37 & 0.06 & 0.59 & 0.05 & 0.07 & 0.02 \\
\hline \multicolumn{9}{|c|}{ TBP } \\
\hline $\begin{array}{l}\text { Cellular } \\
\text { plains }\end{array}$ & 0.005 & 0.22 & 0.21 & 0.04 & 0.54 & 0.02 & 0.04 & 0.01 \\
\hline
\end{tabular}




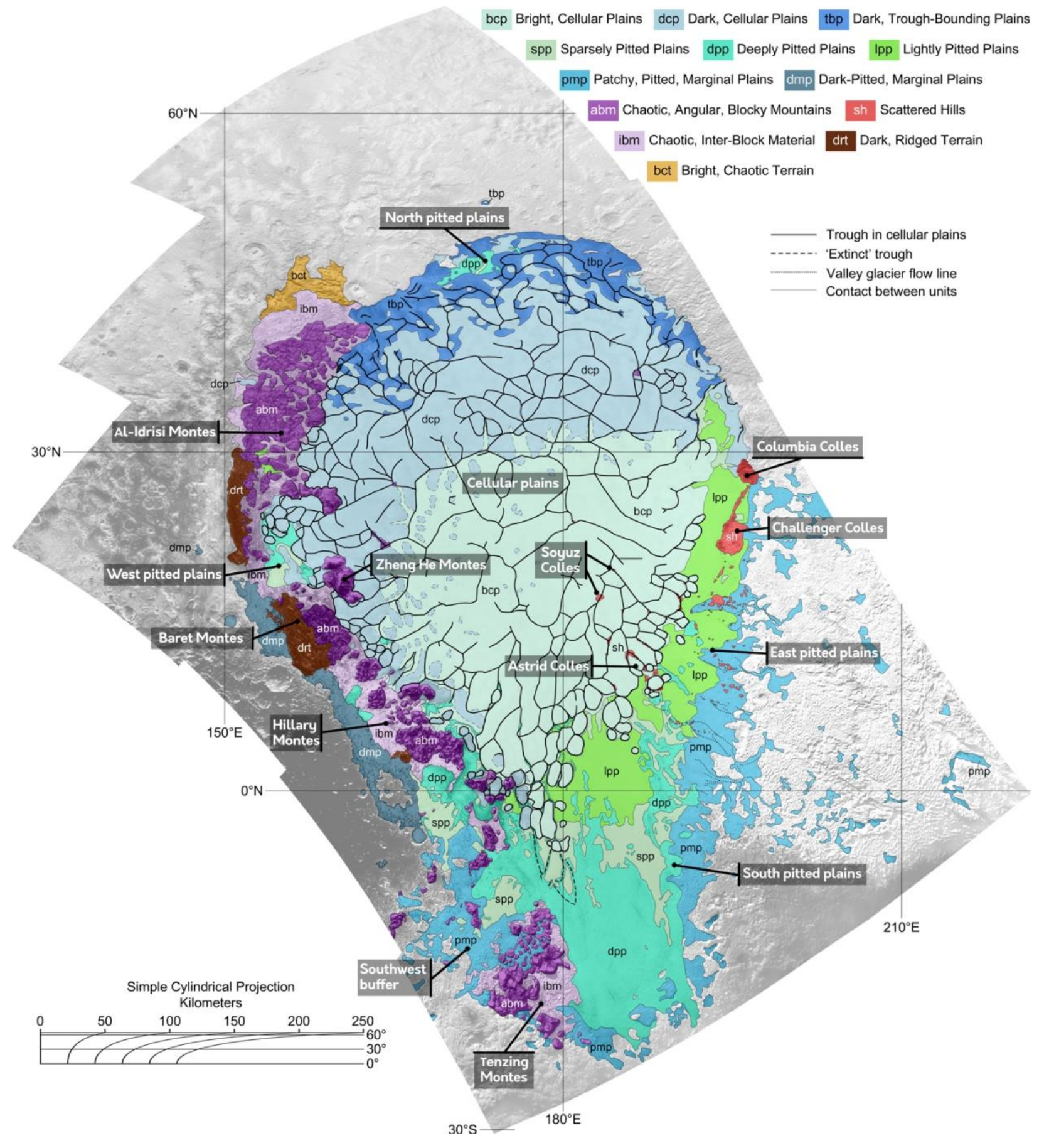

Figure 1: Geologic units identified in Sputnik Planitia by White et al. (2017) through analysis of New Horizons LORRI images. The original 15 units initially identified were reduced to 13 here, to include only the geologic units within and around Sputnik Planitia. The basemap mosaic is a combination of 12 LORRI images, with a spatial resolution of $386 \mathrm{~m} / \mathrm{px}$. 


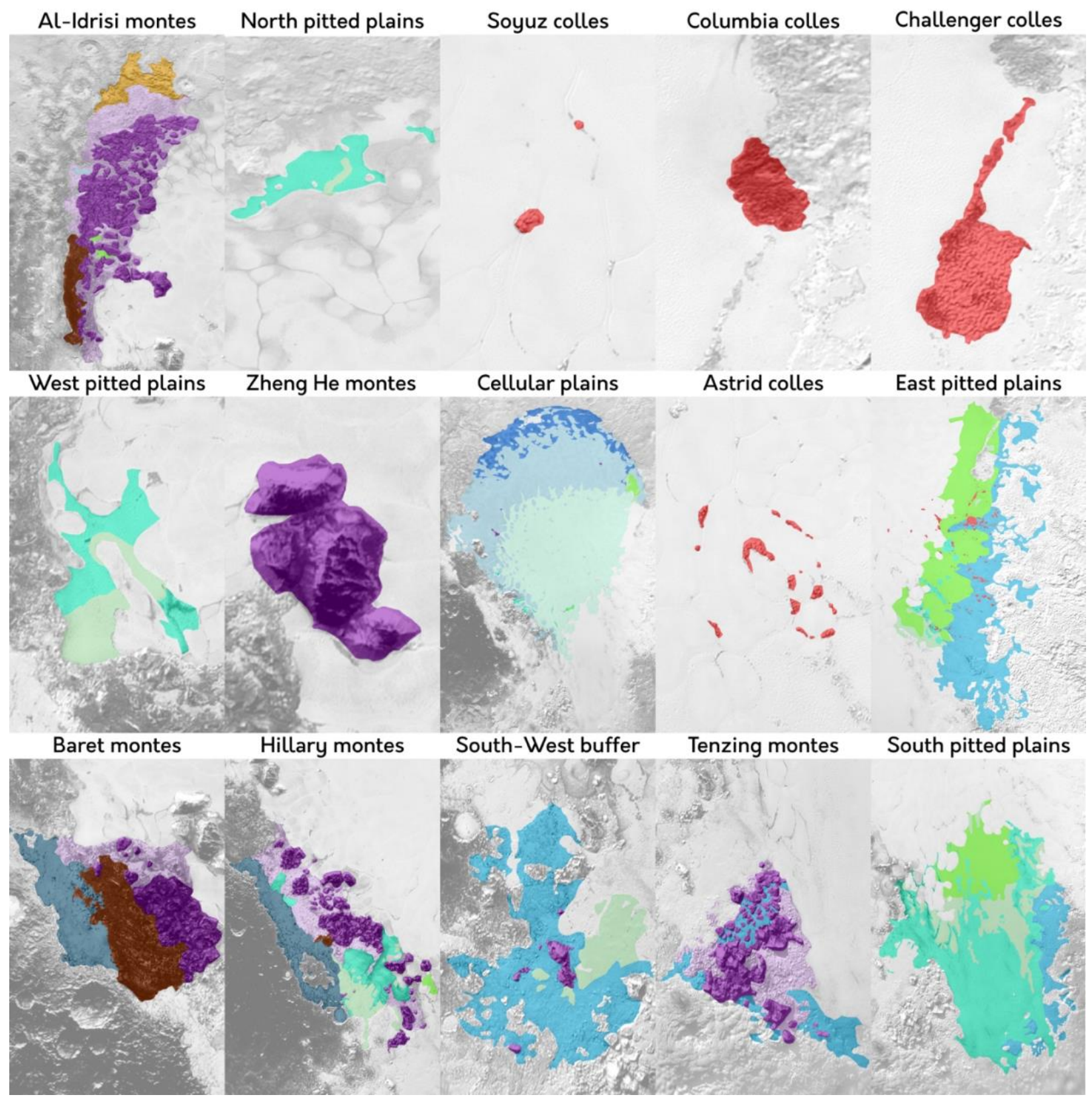

Figure 2: The 15 provinces that we have divided the Sputnik Planitia geologic map into, with each province containing one or more geologic unit. Indeed, some geologic unit covers areas that are spatially distant and surrounded by very divers terrain types. For example, spp unit can be found in 5 different provinces: in the northernmost part of Sputnik Planitia (North Pitted Plains), embedded in the cellular plains; in the West Pitted Plains, again close to the cellular plains, but also to the blocky mountains; in the Hillary Montes region, surrounded by inter-blocky material; in the South-West Buffer and the South-East Pitted Plains, where the other types of pitted plains dominate. 


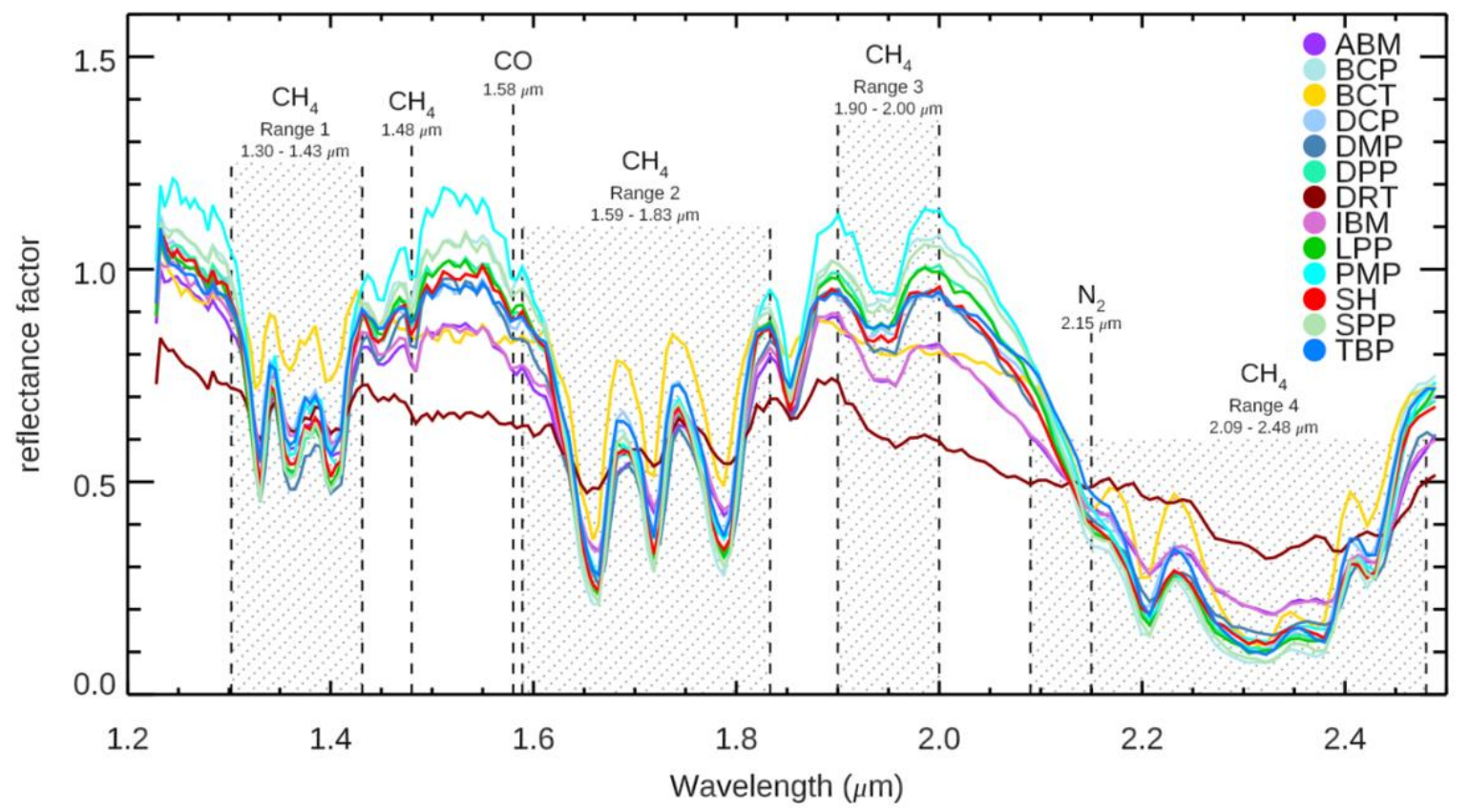

Figure 3: Average spectra of the 13 geologic units identified by White et al. (2017). The boxes indicate the location of the main absorption bands of $\mathrm{CH}_{4}, \mathrm{CO}$ and $\mathrm{N}_{2}$. 


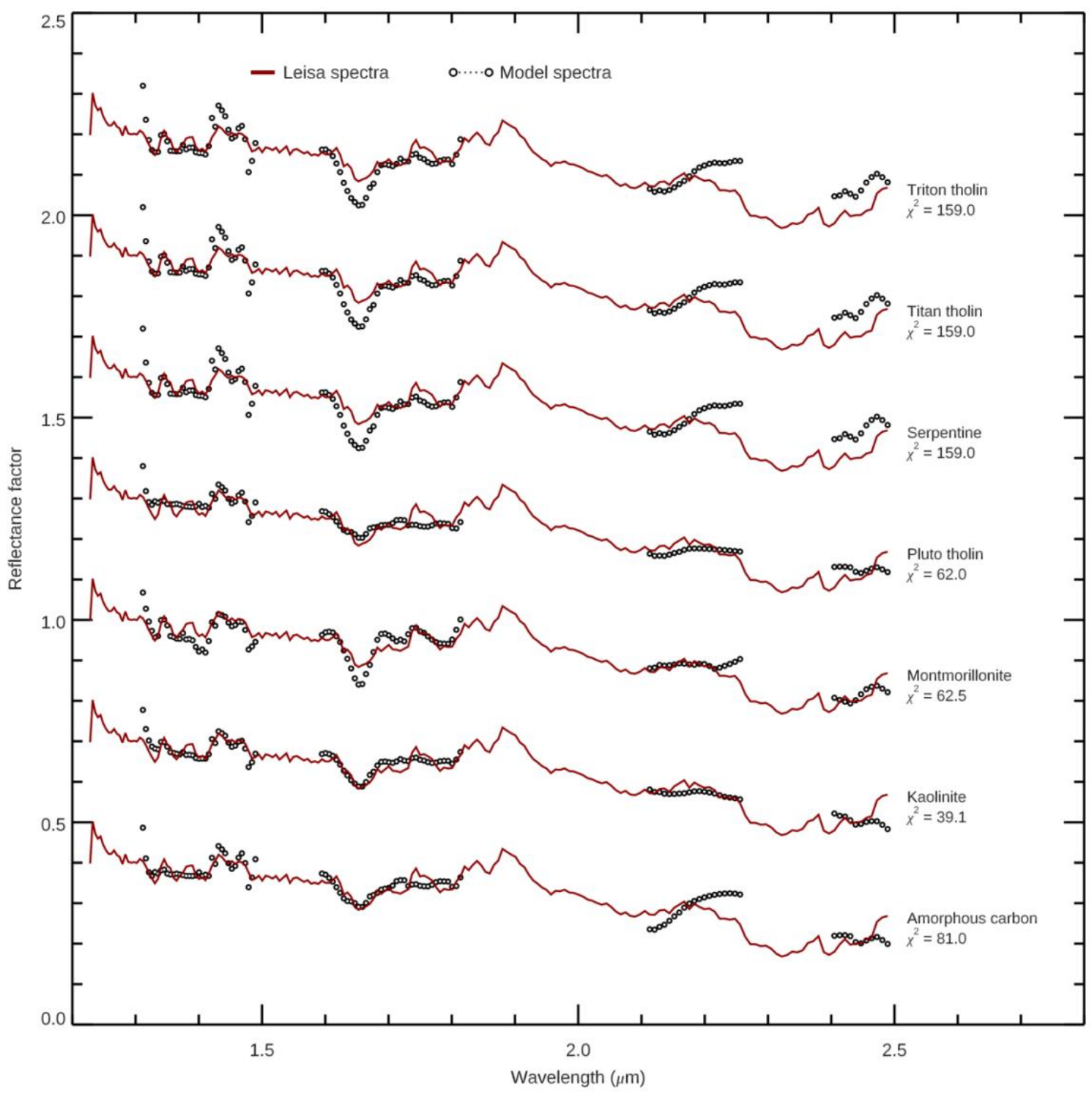

Figure 4: LEISA spectra of unit drt in Baret overlapped by seven Hapke theory-based best fit models, calculated by considering the optical constants of seven different materials to account for the observed negative spectral slope. The spectra are offset for clarity. 


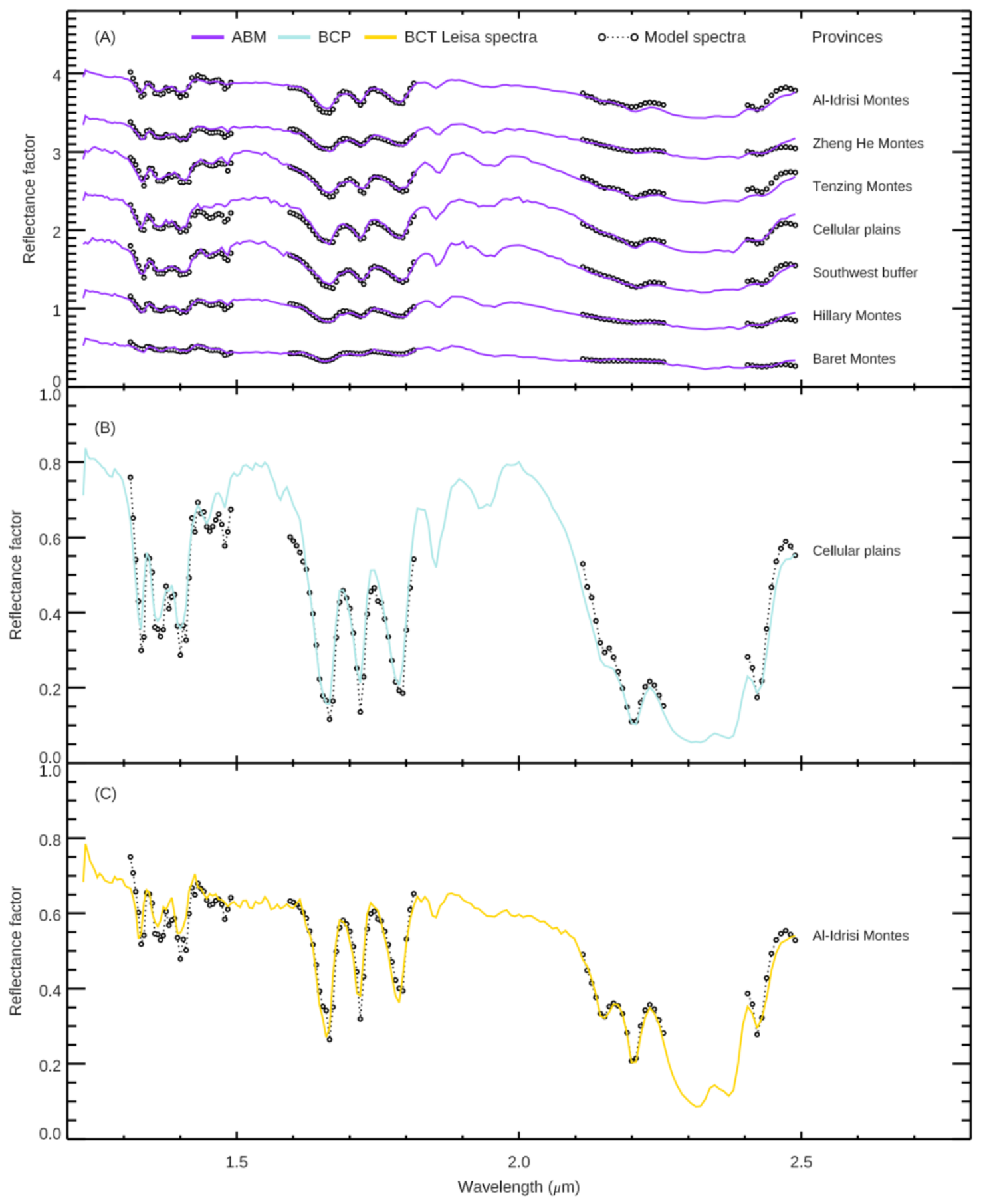




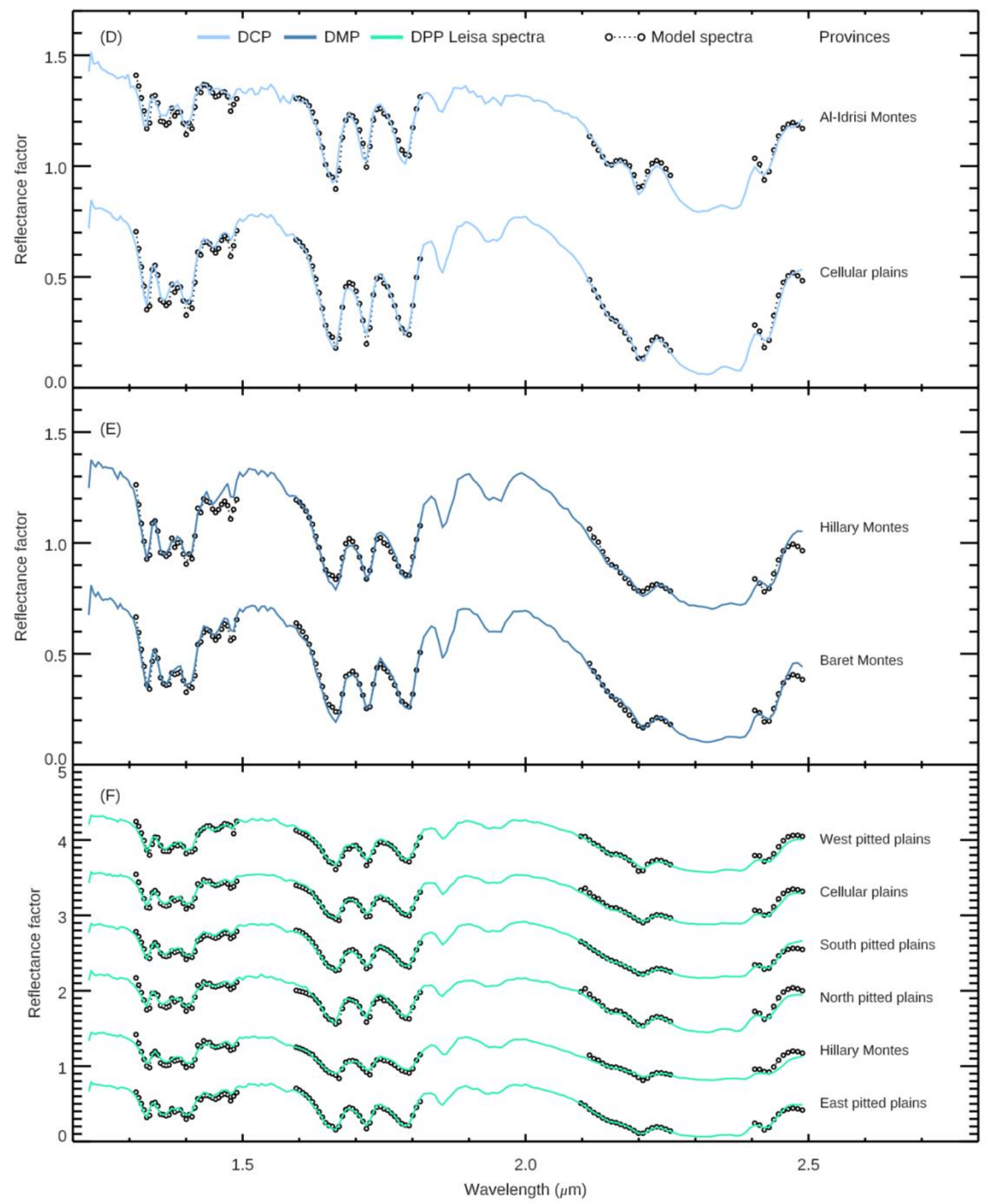




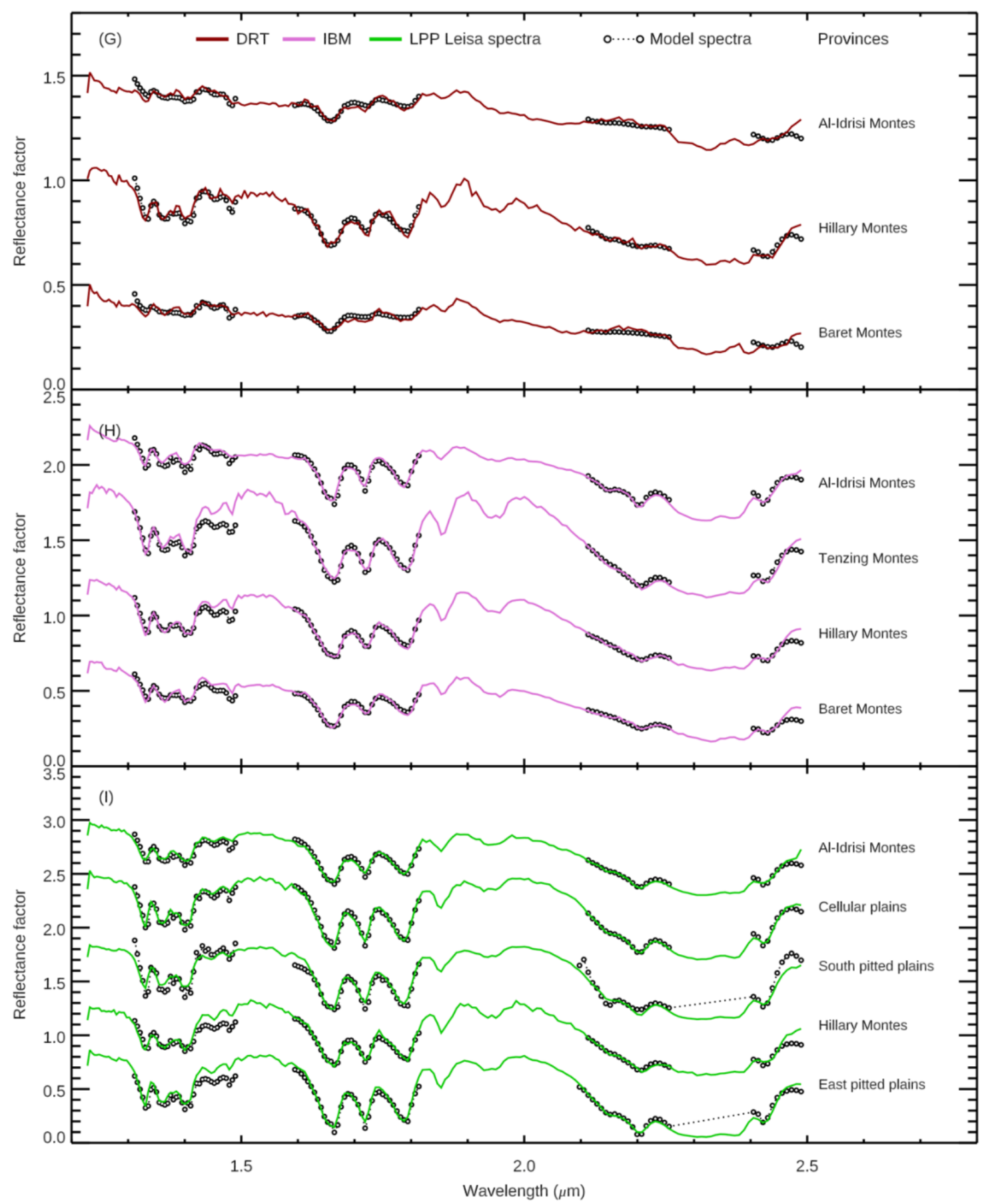




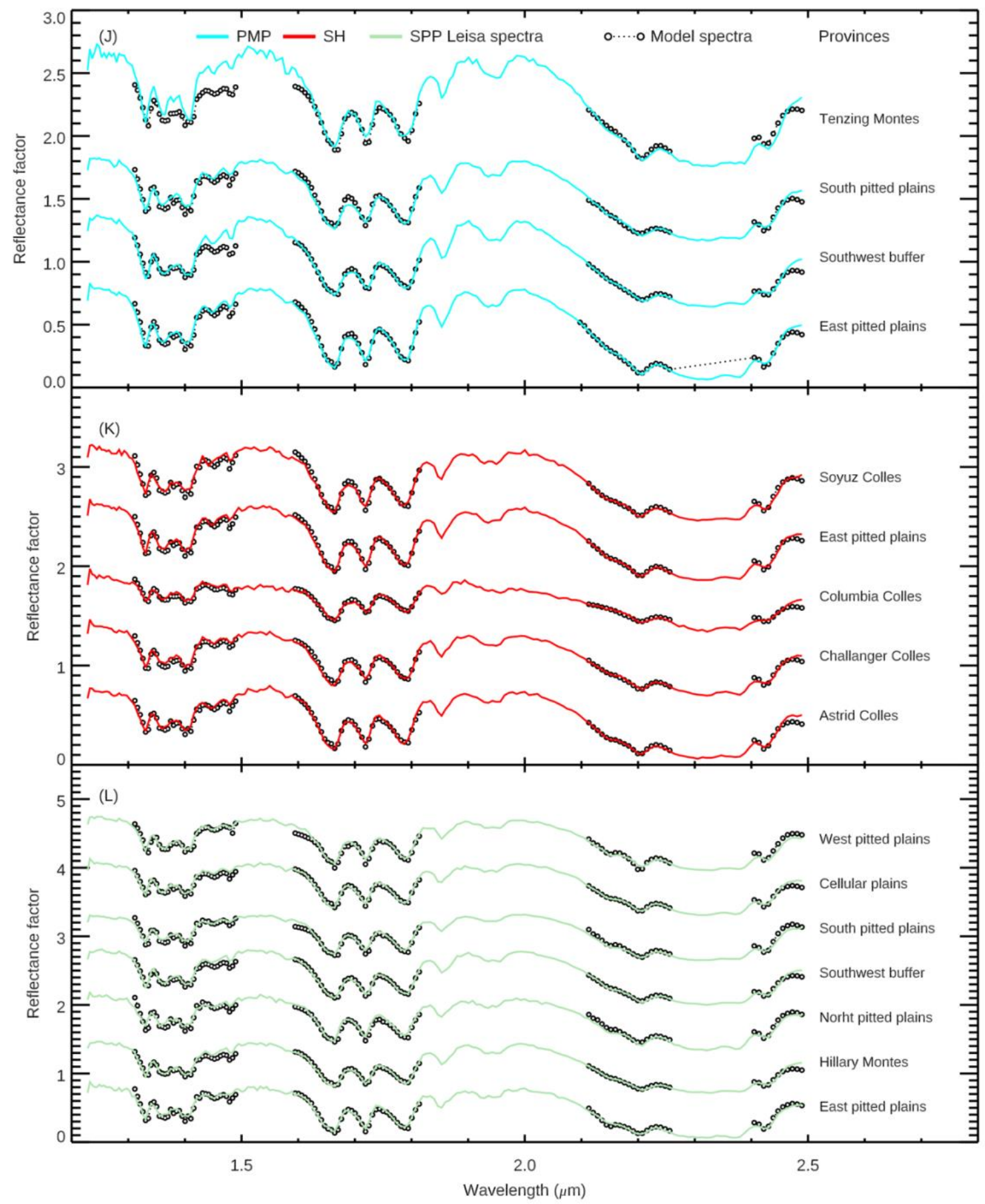




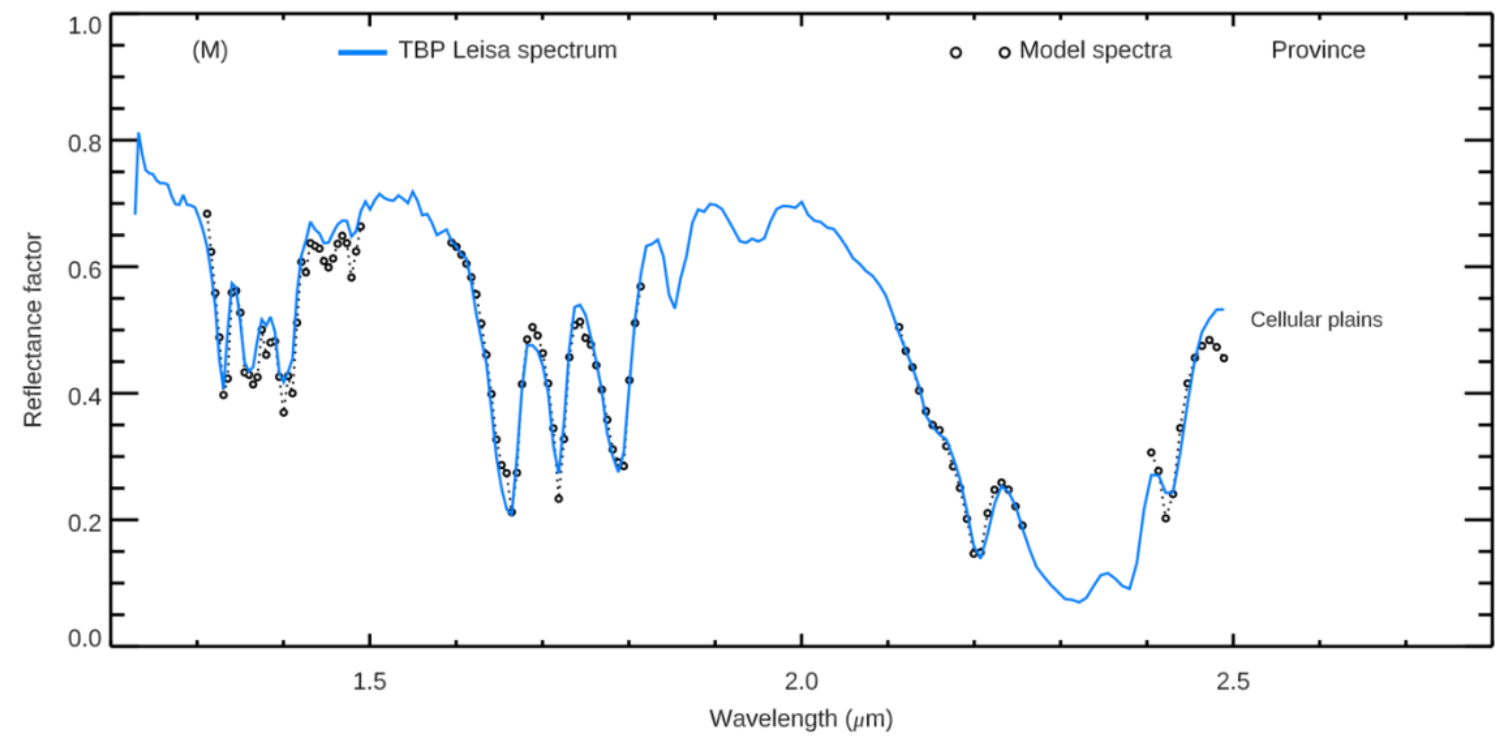

Figure 5A-M: Comparison between LEISA spectra, averaged for each geologic unit in the 15 provinces, and the models calculated by applying the Hapke theory. Each panel also reports the components used in the models, with their fractional area. 


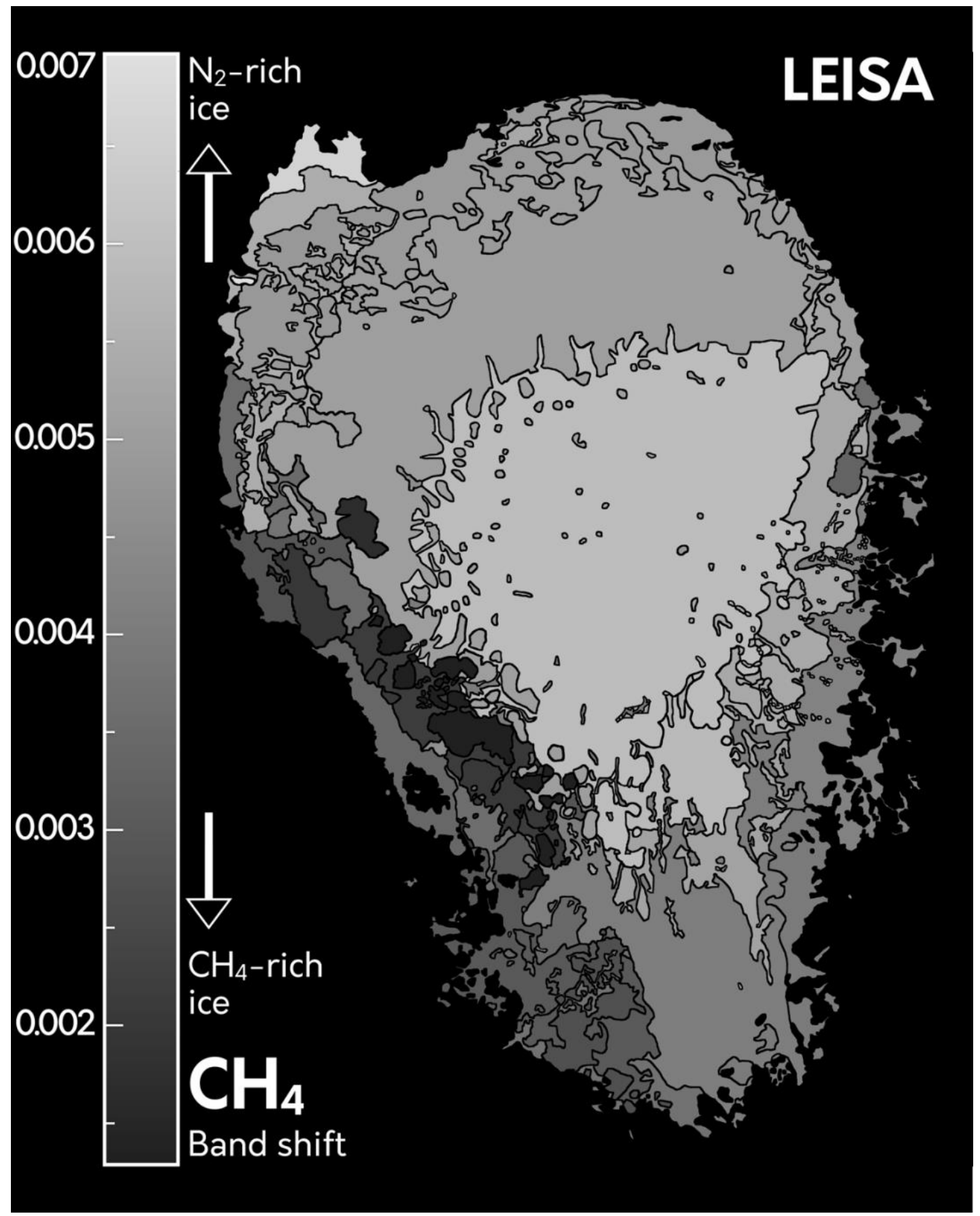

Figure 6: Variation of the $\mathrm{CH}_{4}$ band shift index across the geologic units of Sputnik Planitia. The highest values of the band shift index in the map (light grey) indicate areas characterized by $\mathrm{N}_{2}$-rich ice, while the lowest values (dark grey) indicate $\mathrm{CH}_{4}$-rich ice. 


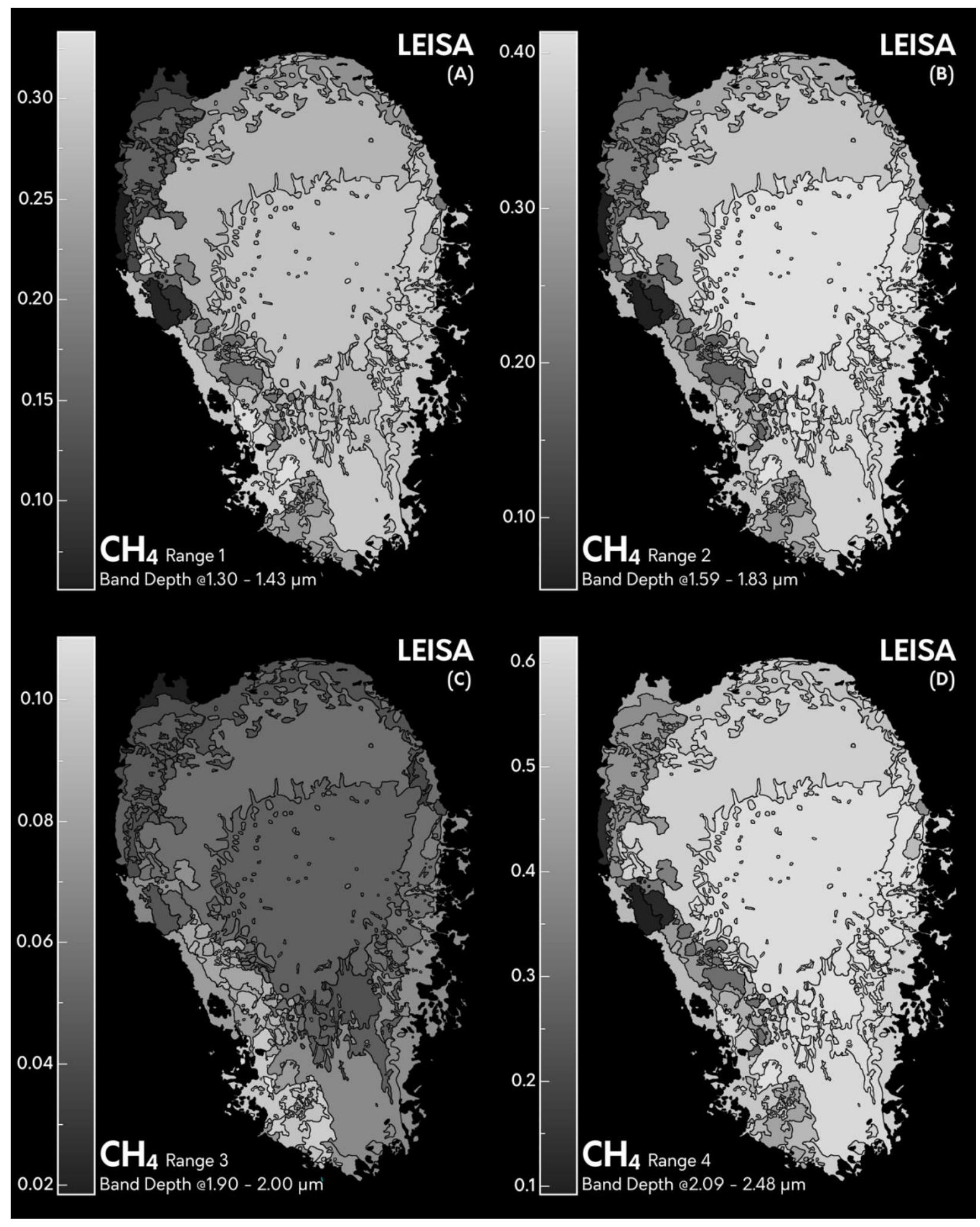




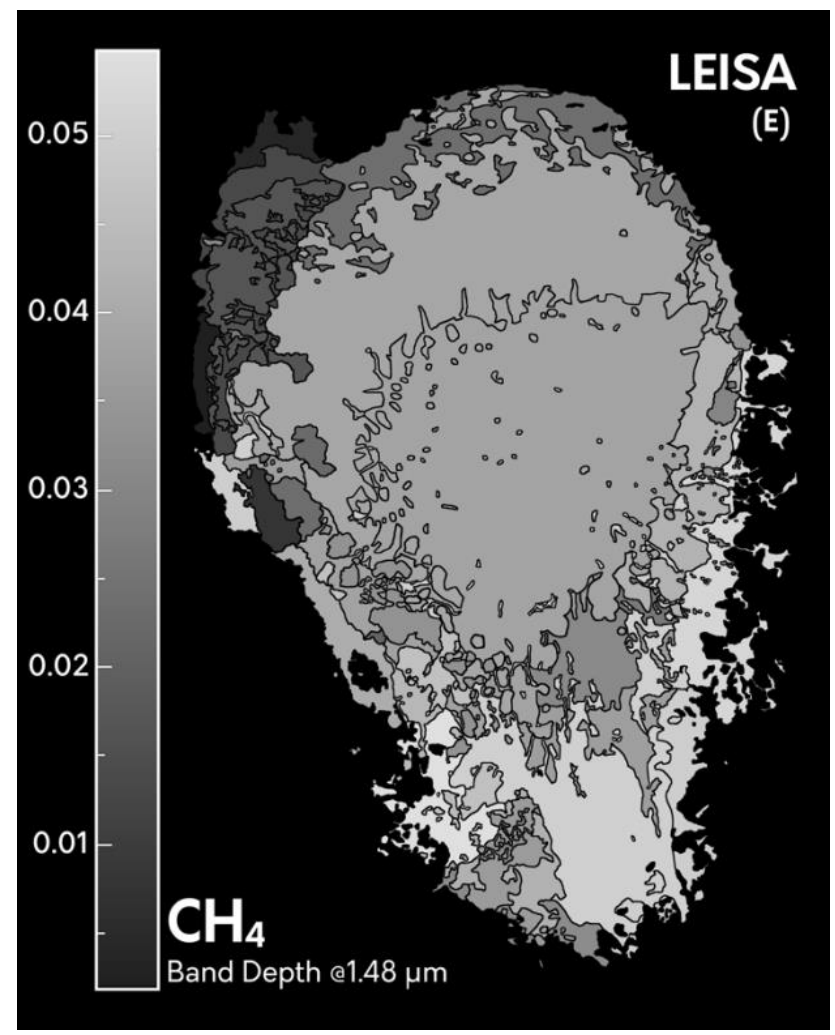

Figure 7 A-E: Maps of variation of the integrated band depths of the main $\mathrm{CH}_{4}$ absorptions: Range 1 (1.30-1.43

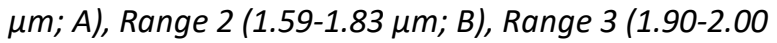
$\mu \mathrm{m} ; \mathrm{C})$, Range 4 (2.09-2.48 $\mu \mathrm{m} ; \mathrm{D})$, and the single band at $1.48 \mu \mathrm{m}$ (E). The Range 3 map (C) shows opposite trends compared to the other four spectral ranges, a behavior that was previously observed also in other studies (e.g. Schmitt et al, 2017).

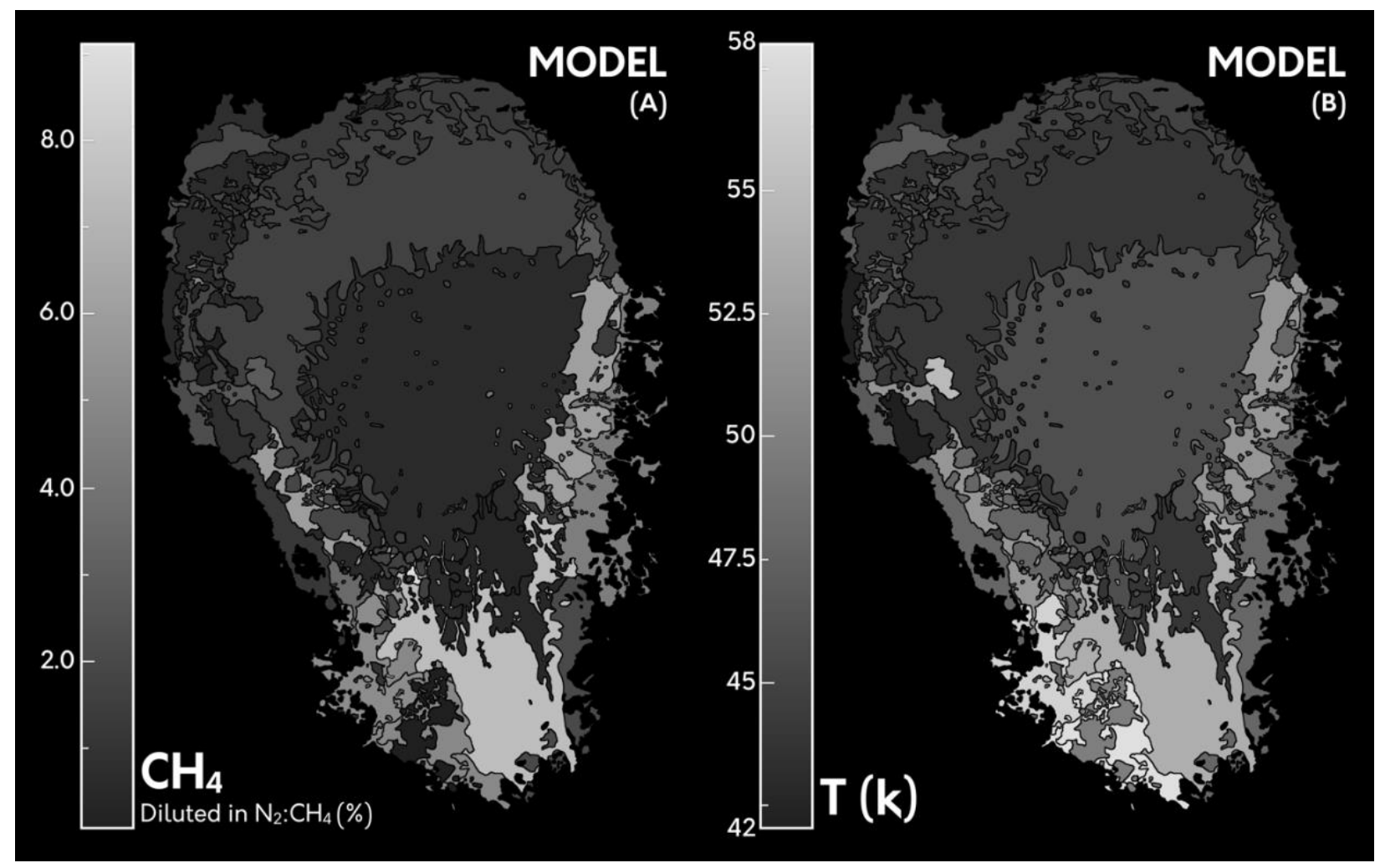

Figure 8A-B: Maps of percentage variation of $\mathrm{CH}_{4}$ diluted in $\mathrm{N}_{2}$-rich ice $(A)$ and temperatures $(B)$ estimated by the spectral modeling. 


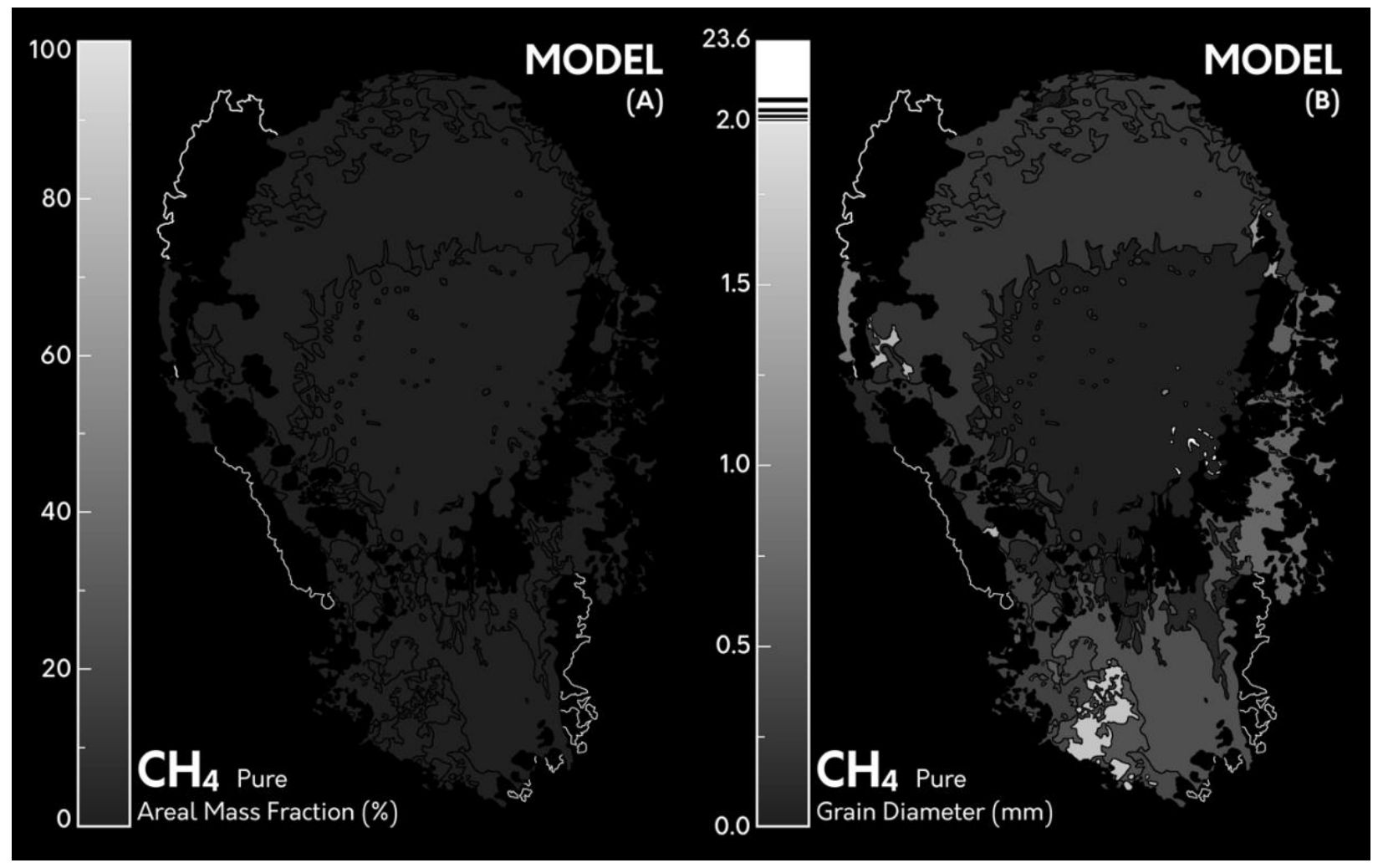

Figure 9A-B: Maps of variation of pure $\mathrm{CH} 4$ areal mass fraction (A) and grain size (A) estimated by the spectral modeling. Pure $\mathrm{CH} 4$ covers almost all geologic units in Sputnik Planitia, but its percentage is very low, below 1.5\%. 


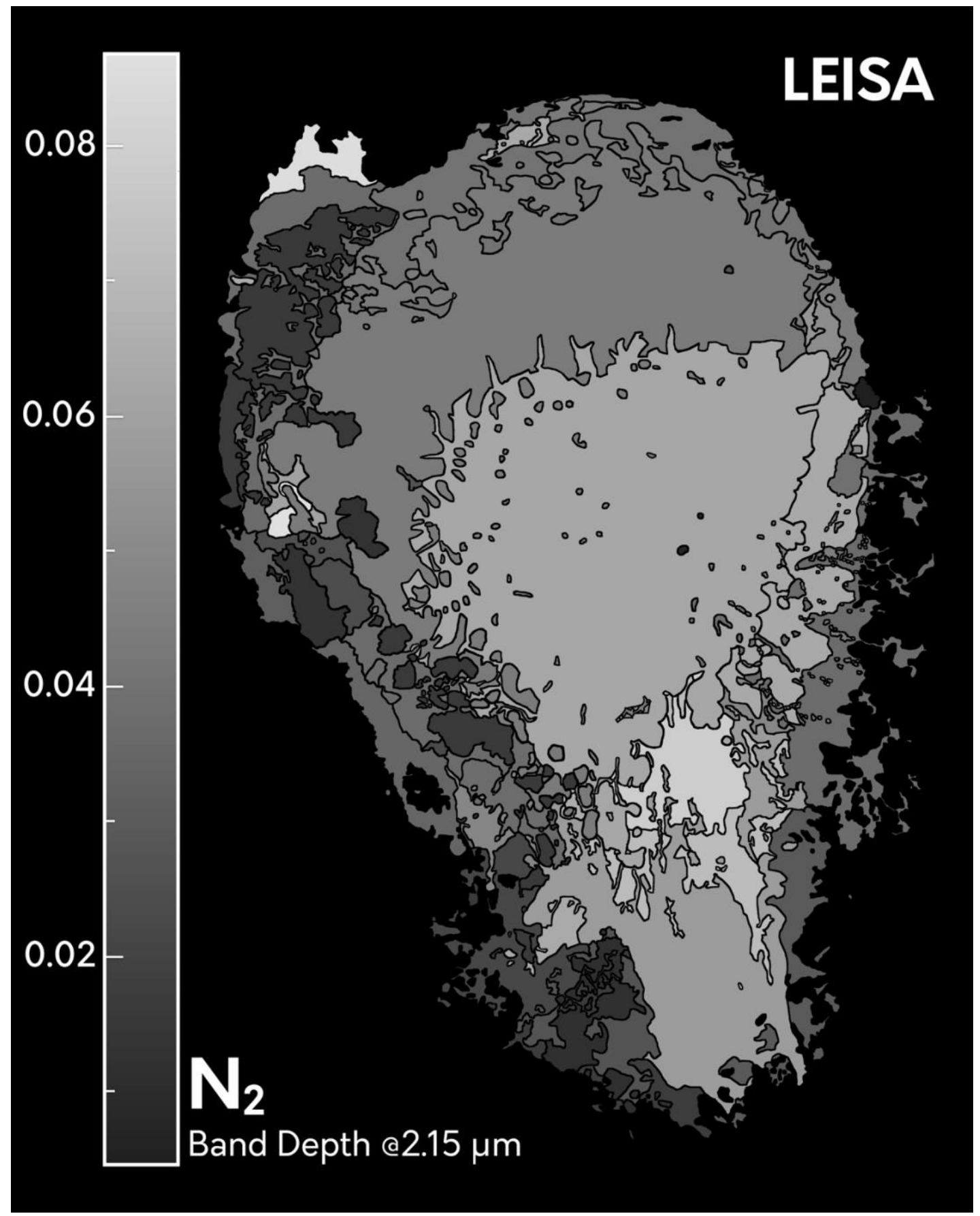

Figure 10: Map of variation of the integrated band depth of $\mathrm{N}_{2}$ at $2.15 \mu \mathrm{m}$. 


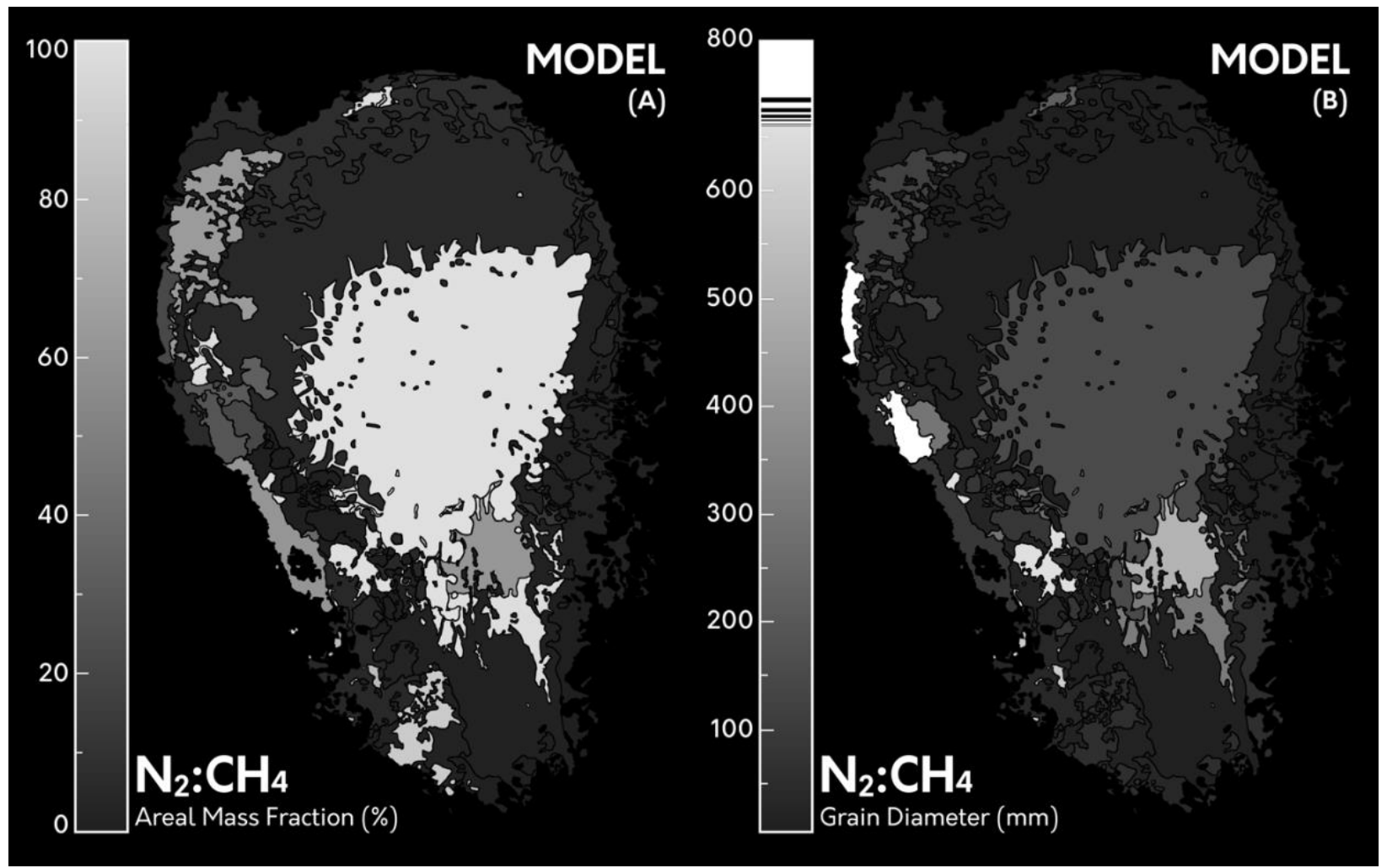

Figure 11A-B: Maps of variation of $\mathrm{N}_{2}: \mathrm{CH}_{4}$ areal mass fraction (A) and grain size (B) estimated by the spectral modeling.

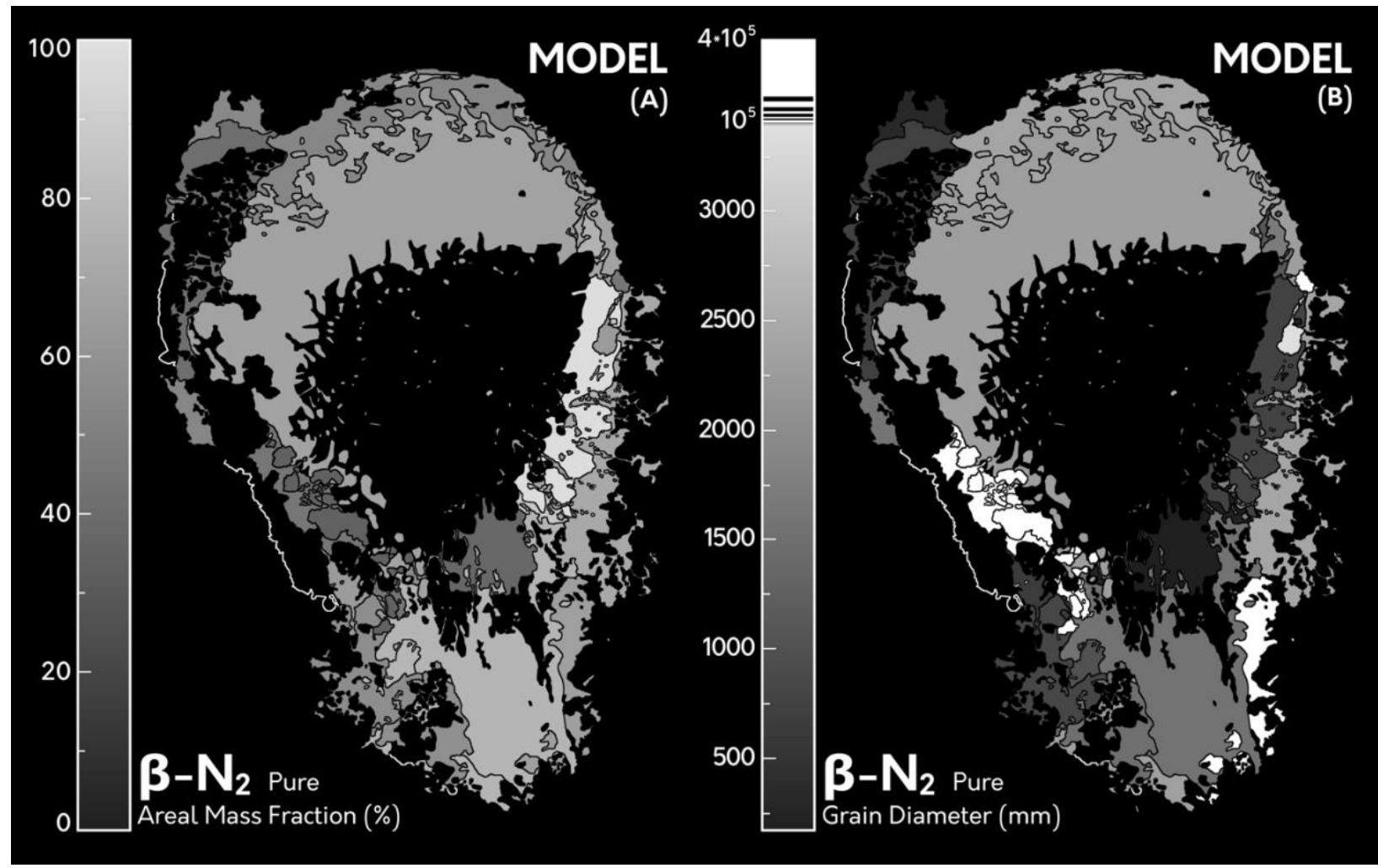

Figure 12A-B: Maps of variation of pure $B-N_{2}$ areal mass fraction $(A)$ and grain size $(B)$ estimated in the spectral modeling. 

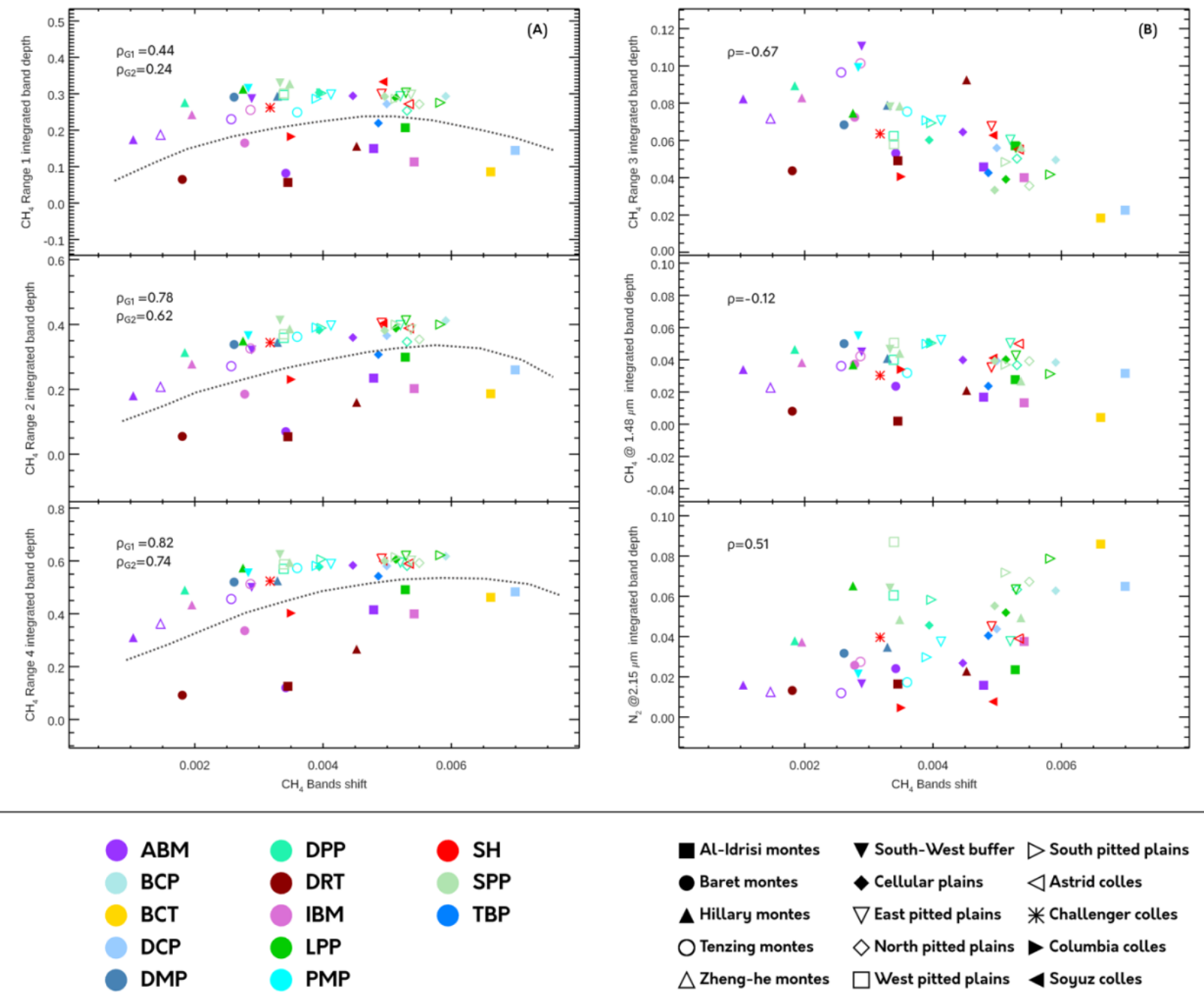

Figure 13: (A) Scatter plot showing the correlation between the CH4 integrated band depth in Ranges 1 (top), 2 (middle), and 4 (bottom) with the CH4 shift index. The data are split into two groups, separated by a dotted curve. The points belonging to group G1, above the curve, show increasing band strength with increasing values of the shift parameter. The second group (G2), below the dotted line, also shows a positive trend, but the points are more dispersed. Furthermore, the values of the $\mathrm{CH} 4$ integrated band depths in $\mathrm{G} 2$ are lower than the units in $\mathrm{G} 1$. The positive trends in both groups contradicts the definition of the $\mathrm{CH} 4$ shift index, i.e. higher index values correspond to lower of CH4-rich ice amount. Therefore, a trend opposite of what observed in panel $\mathrm{A}$ is expected: the $\mathrm{CH} 4$ integrated band depth should be less intense - or, the abundance of methane should decrease - with increasing values of the shift index. (B) Scatter plot showing the correlation between the CH4 integrated band depth in Range 3 (top), at $1.48 \mu \mathrm{m}$ (middle), and the $\mathrm{N} 2$ integrated band depth at $2.15 \mu \mathrm{m}$ (bottom) with the $\mathrm{CH} 4$ shift index. As opposite to the three plots in panel $A$, these spectral parameters show a clear and single trend with the $\mathrm{CH} 4$ shift index, in agreement with the definition of the $\mathrm{CH} 4$ shift index. 


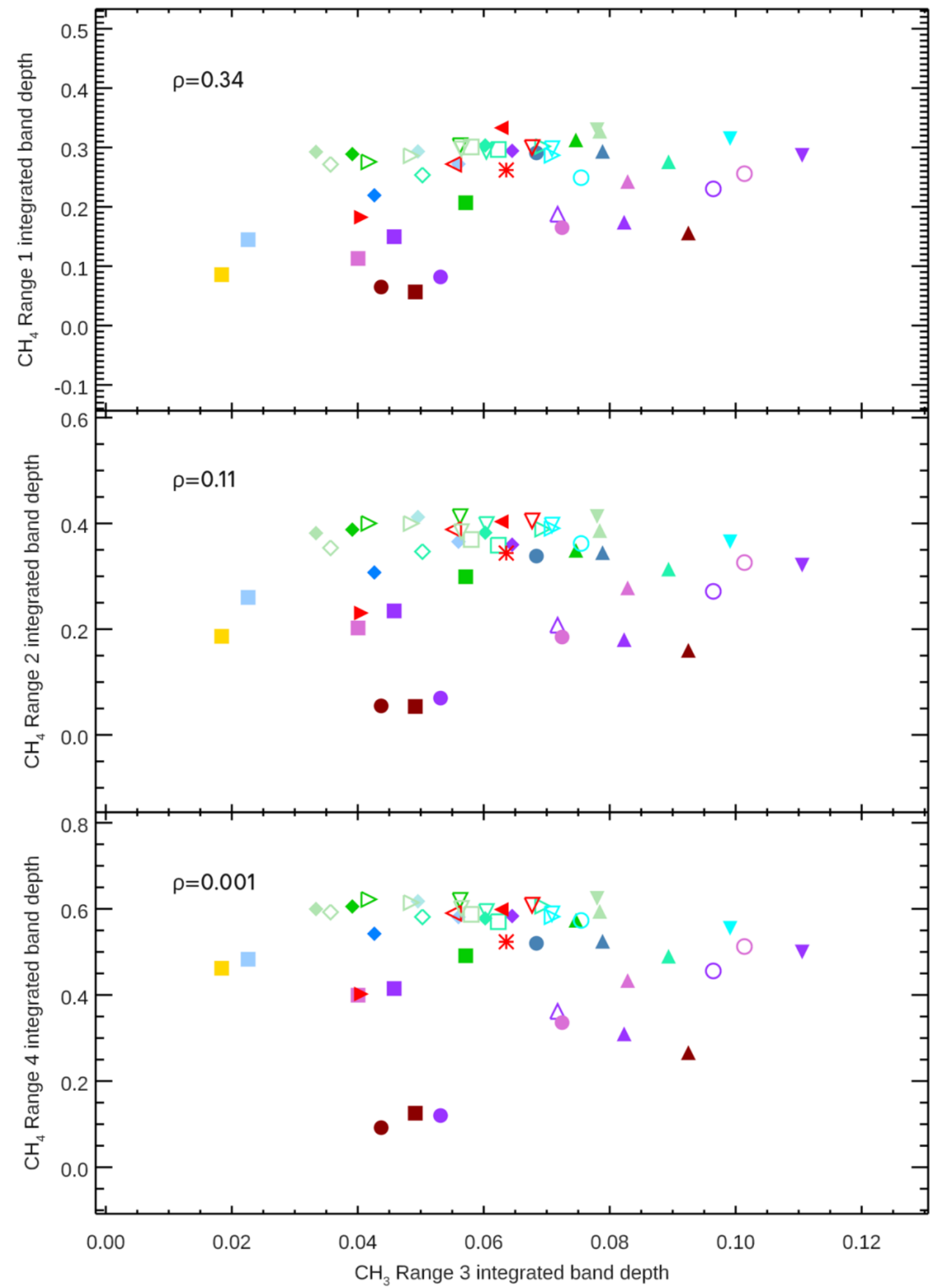

Figure 14: Scatter plot showing the correlation between the CH4 integrated band depths in Ranges 1 (top), 2 (middle), and 4 (bottom) versus the $\mathrm{CH} 4$ integrated band depth in Range 3 . The plots clearly show a lack of correlation between the represented parameters, indicating that the factor influencing the depth of the $\mathrm{CH} 4$ absorptions in Ranges 1, 2, and 4 doesn't have any (or it has negligible) effect on the absorption in Range 3. 


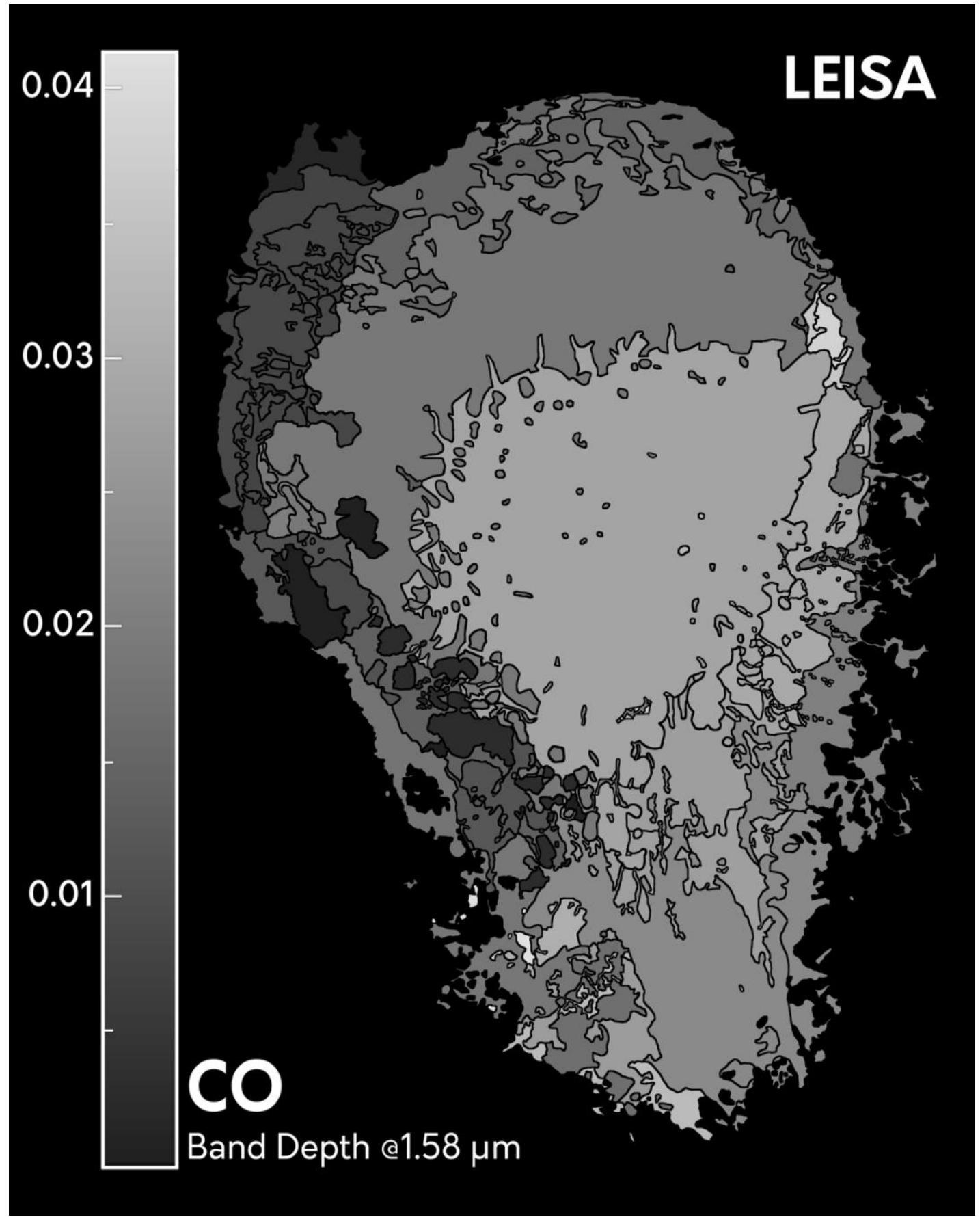

Figure 15: Maps of variation of CO integrated band depth at $1.58 \mu \mathrm{m}$. 

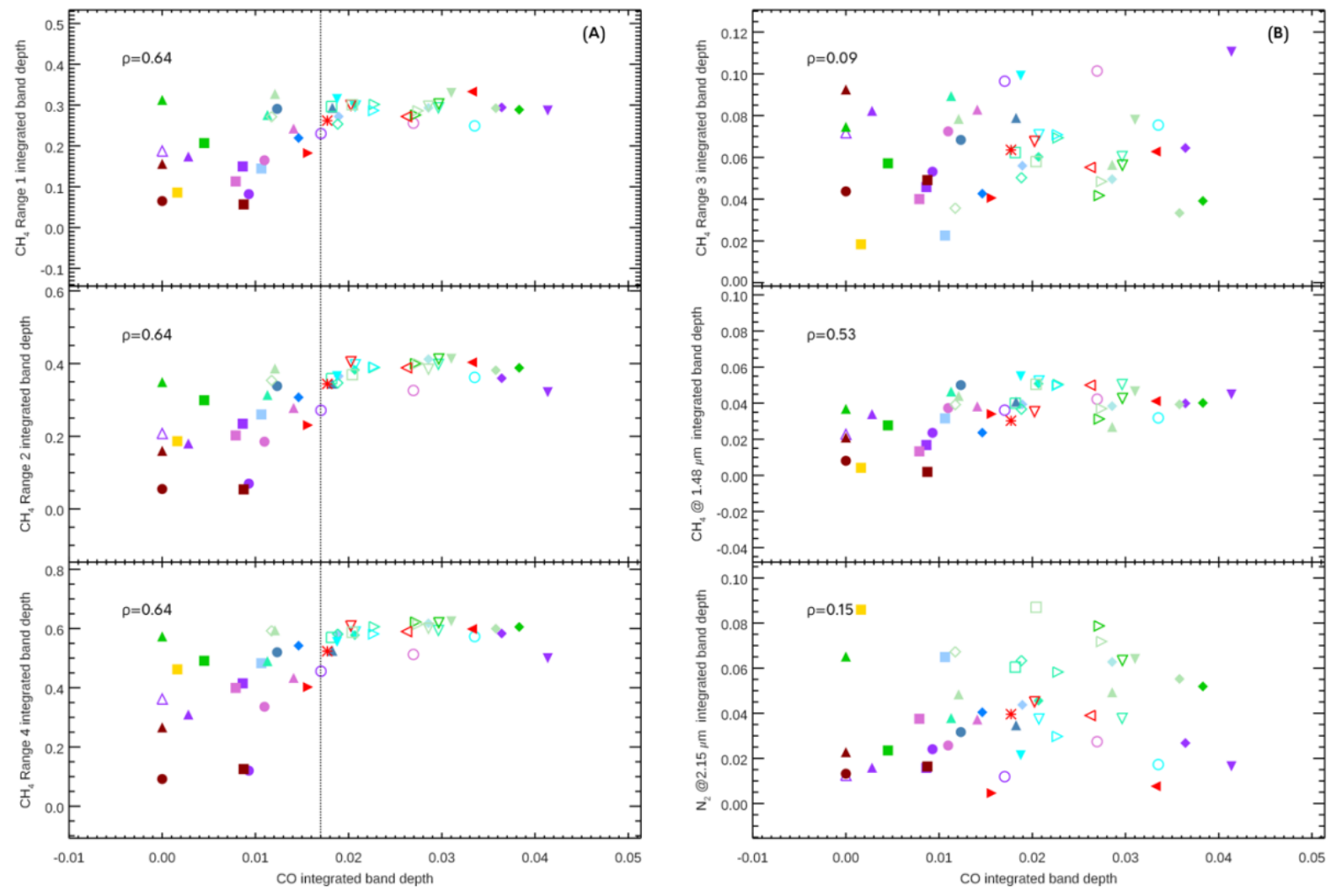

$\begin{array}{lll}\text { ABM } & \text { DPP } & \text { SH } \\ \text { BCP } & \text { DRT } & \text { SPP } \\ \text { BCT } & \text { IBM } & \text { TBP } \\ \text { DCP } & \text { LPP } & \\ \text { DMP } & \text { PMP } & \end{array}$

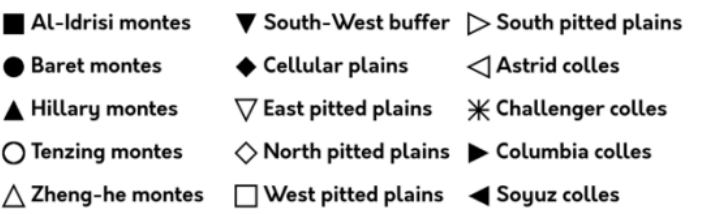

Figure 16: (A) Scatter plot showing the correlation between the $\mathrm{CH}_{4}$ integrated band depth in Ranges 1 (top), 2 (middle), and 4 (bottom) with the CO integrated band depth. Because rhe data shows to different trends, we split them into two groups, separated by a vertical dotted line. The units on the left side have a positive trend but a sparse distribution, the points on the right portion of the plots show a flat distribution. (B) Scatter plot showing the correlation between the $\mathrm{CH}_{4}$ integrated band depth in Range 3 (top), at $1.48 \mu \mathrm{m}$ (middle), and the $\mathrm{N}_{2}$ integrated band depth at $2.15 \mu \mathrm{m}$ (bottom) with the $\mathrm{CO}$ integrated band depth. The only clear correlation occurs between $\mathrm{CO}$ and the band at $1.48 \mu \mathrm{m}$, while the correlation coefficient for $\mathrm{CH}_{4}$ band in Range 3 and $\mathrm{N}_{2}$ are negligible. 


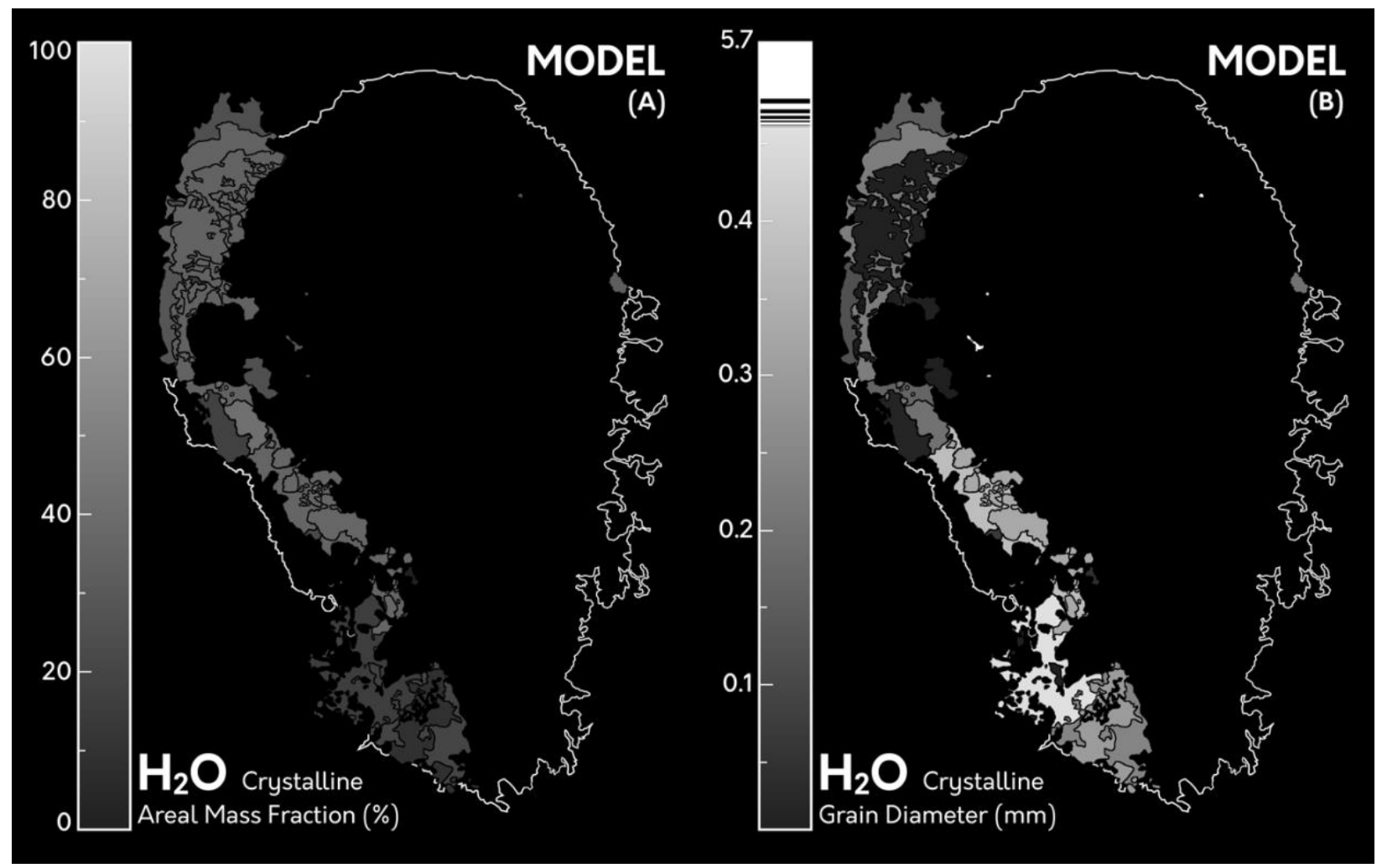

Figure 17A-B: Maps of variation of Crystalline $\mathrm{H}_{2} \mathrm{O}$ areal mass fraction (A) and grain size(B) estimated by the spectral modeling 

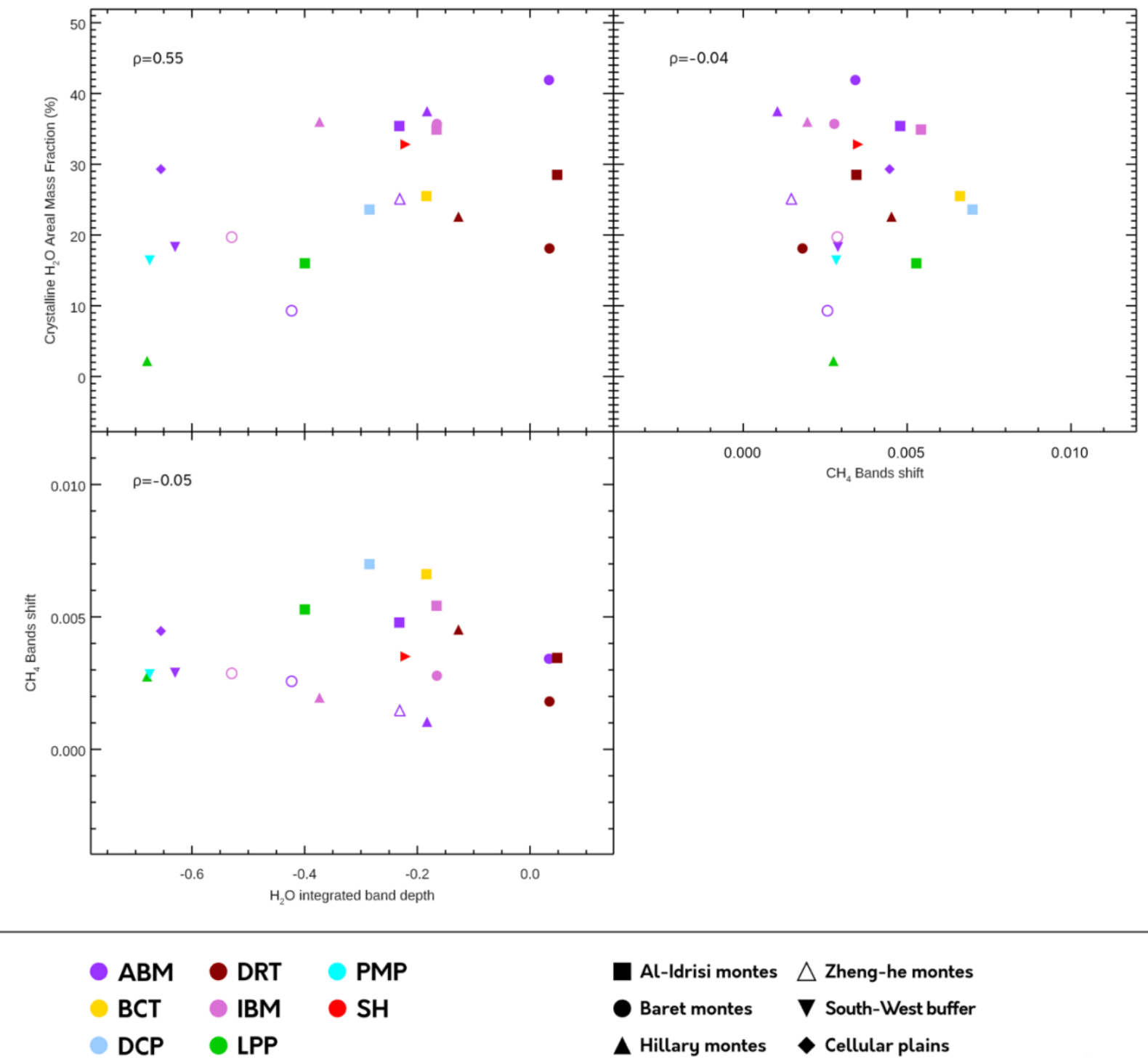

Al-Idrisi montes $\triangle$ Zheng-he montes

Baret montes $\quad \nabla$ South-West buffer

$\Delta$ Hillary montes Cellular plains

Tenzing montes Columbia colles

Figure 18: Mutual variation of the crystalline $\mathrm{H}_{2} \mathrm{O}$ areal mass fraction, the $\mathrm{H}_{2} \mathrm{O}$ integrated band depth, and the $\mathrm{CH}_{4}$ shift index. Crystalline $\mathrm{H}_{2} \mathrm{O}$ ice well correlates with the $\mathrm{H}_{2} \mathrm{O}$ integrated band depth (top, left panel), while no correlation exists between the $\mathrm{H}_{2} \mathrm{O}$ areal mass fraction (top, right panel) and the $\mathrm{H}_{2} \mathrm{O}$ integrated band depth (bottom, left panel) and the shift parameter, suggesting that $\mathrm{H}_{2} \mathrm{O}$ do not influence the $\mathrm{CH}_{4}$ band shift. 


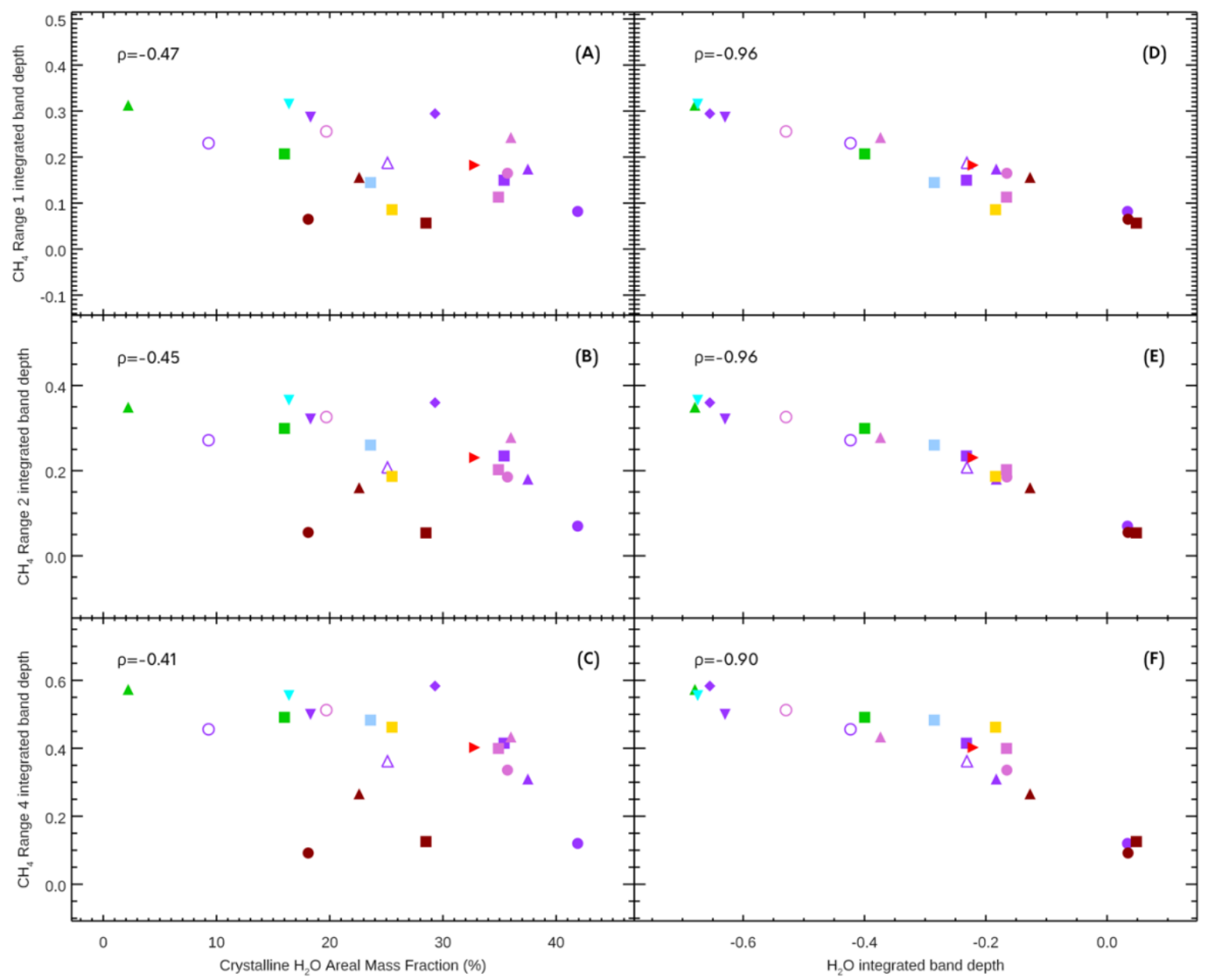

Figure 19: (A-F) Scatter plot showing the correlation between the $\mathrm{CH}_{4}$ integrated band depth in Ranges 1 (top), 2 (middle), and 4 (bottom) with the $\mathrm{H} 2 \mathrm{O}$ crystalline areal mass fraction $(A, B, C)$, and with the $\mathrm{H}_{2} \mathrm{O}$ integrated band depth $(D, E, F)$. All the represented parameters show clear anti-correlations, suggesting that water ice can contribute to the suppression of the strong methane ice absorptions. For some units, though, $\mathrm{H}_{2} \mathrm{O}$ is not the only, or the main, contributor. Unit drt in Al-Idrisi and Baret Montes, and unit abm in Zheng He and Hillary Montes have the strongest absorptions in the $2.0 \mu \mathrm{m}$ region, but moderate fractions of crystalline water ice. The discrepancy between the two water ice parameters suggests that crystalline $\mathrm{H}_{2} \mathrm{O}$ is contributing to the absorption around 2.0 $\mu \mathrm{m}$, but the influence of some other material must be invoked to justify such deep absorptions in this spectral area. 

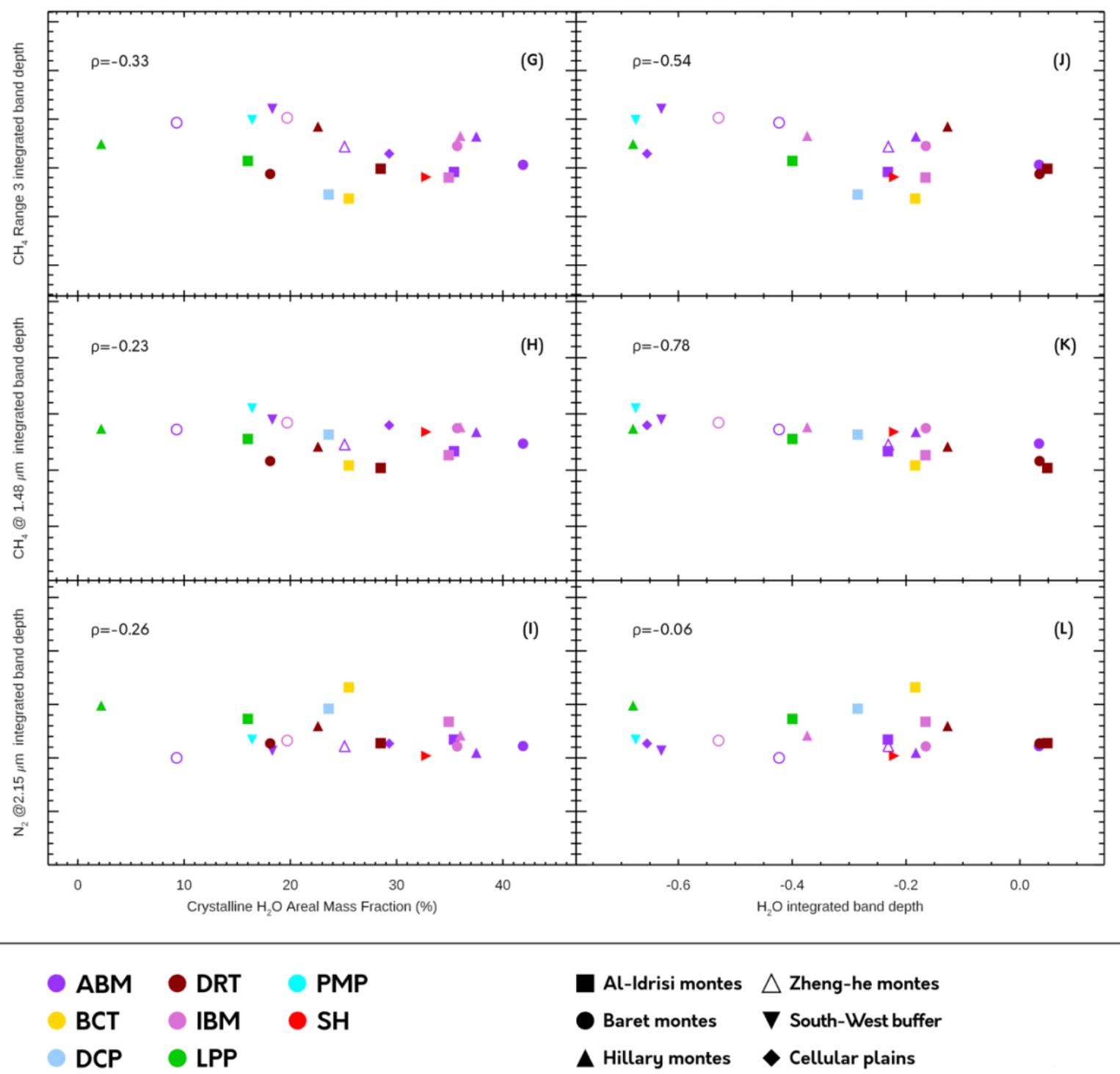

Al-Idrisi montes $\triangle$ Zheng-he montes

Baret montes $\quad \nabla$ South-West buffer

$\Delta$ Hillary montes Cellular plains

Tenzing montes Columbia colles

Figure 19: (G-L) Scatter plot showing the correlation between the $\mathrm{CH}_{4}$ integrated band depth in Ranges 3 (top), at $1.48 \mu \mathrm{m}$ (middle), and $\mathrm{N}_{2}$ integrated band depth at $2.15 \mu \mathrm{m}$ (bottom) with the $\mathrm{H}_{2} \mathrm{O}$ crystalline areal mass fraction $(G, H, I)$, and with the $\mathrm{H}_{2} \mathrm{O}$ integrated band depth $(J, K, L)$. The correlation between these parameters is less pronounced than in the previous panels, and indication that crystalline $\mathrm{H}_{2} \mathrm{O}$ minimally influence the depth of the $\mathrm{CH}_{4}$ absorptions in Range 3 and at $1.48 \mu \mathrm{m}$, and of $\mathrm{N}_{2}$. 


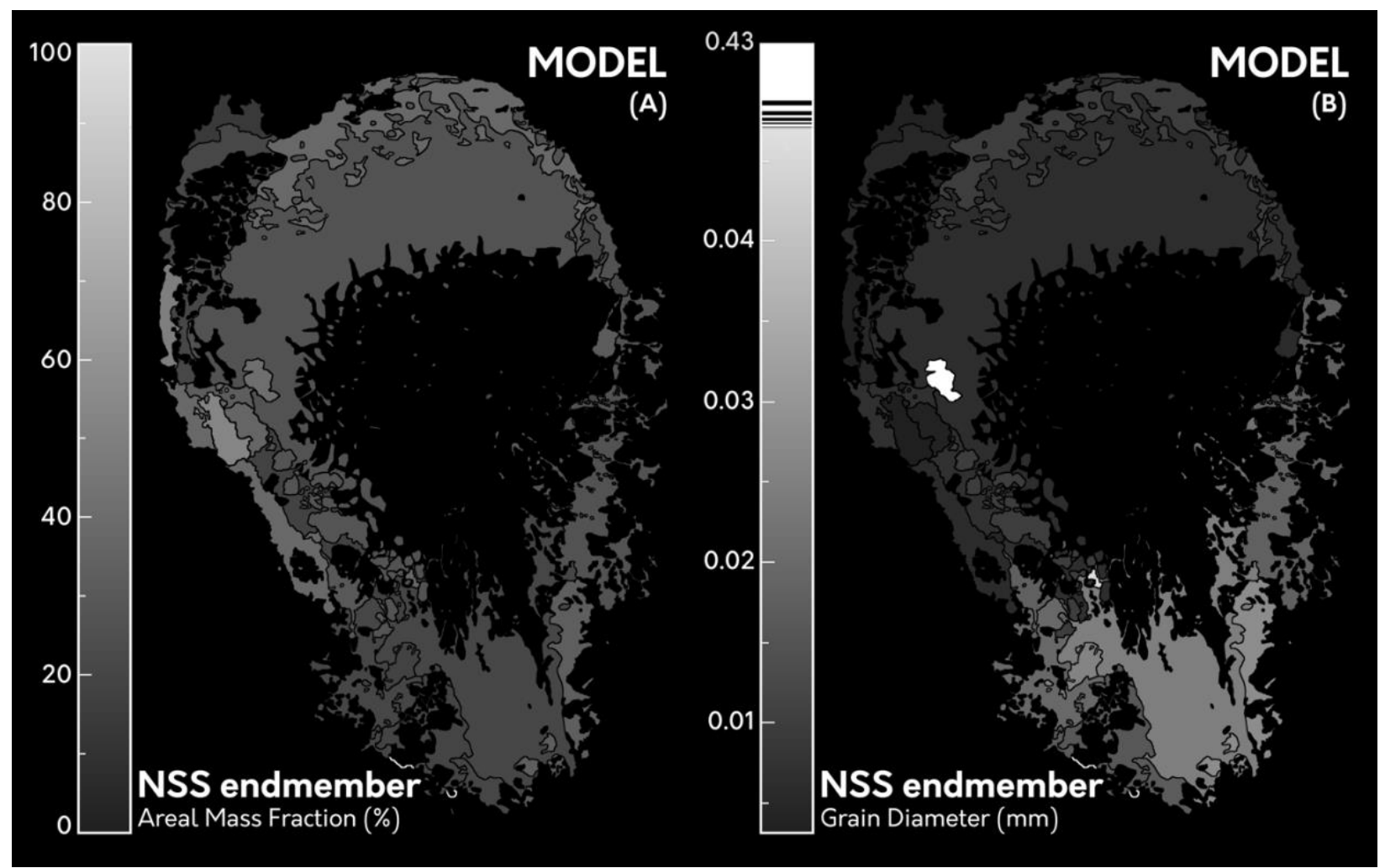

Figure 20: Maps of variation of NSS endmember areal mass fraction (A) and grain size (B) estimated by the spectral modeling. 

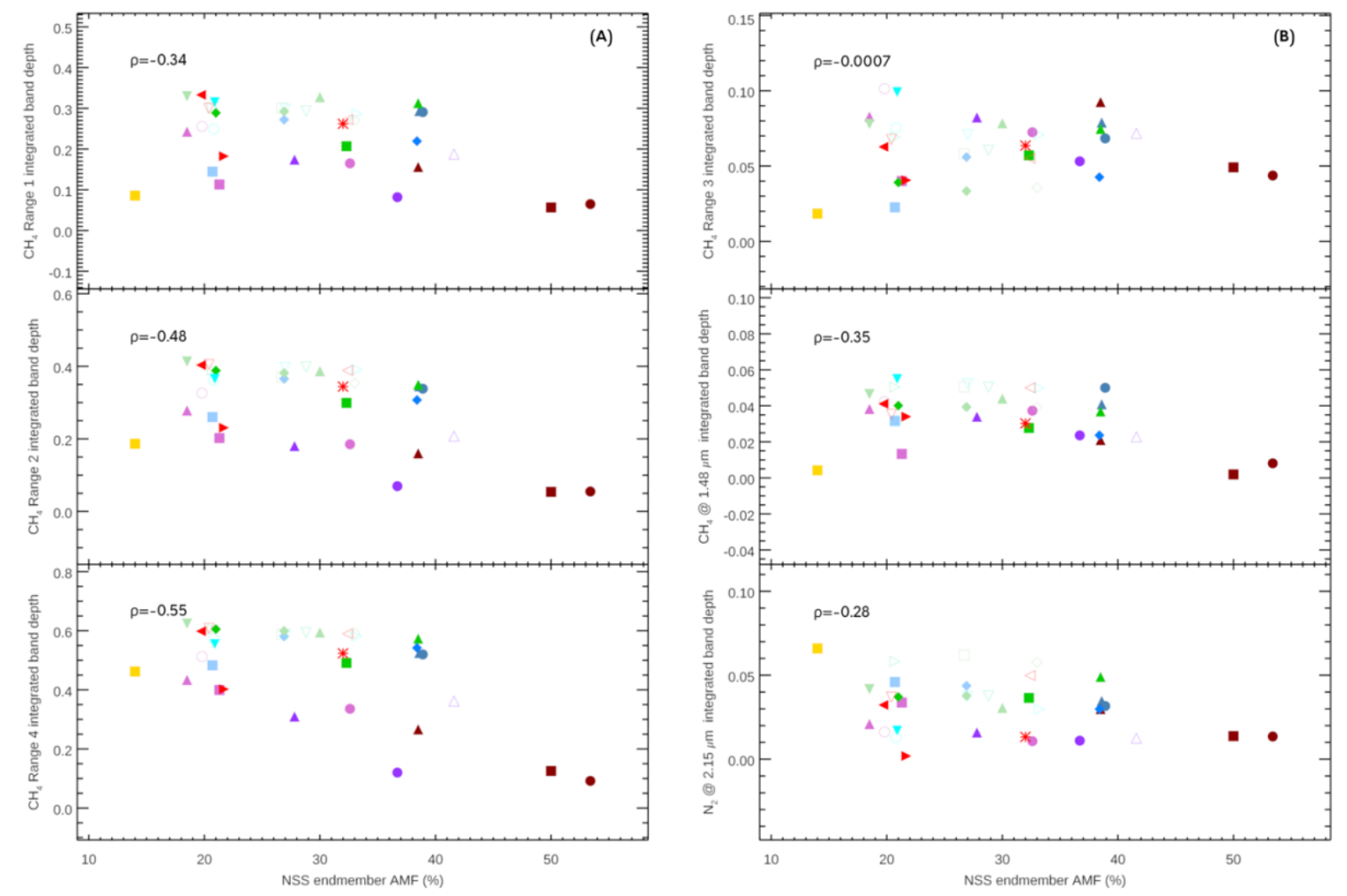

$\begin{array}{lll}\text { ABM } & \text { DPP } & \text { SH } \\ \text { BCP } & \text { DRT } & \text { SPP } \\ \text { BCT } & \text { IBM } & \text { TBP } \\ \text { DCP } & \text { LPP } & \\ \text { DMP } & \text { PMP } & \end{array}$

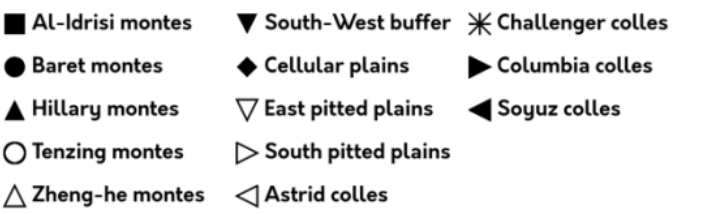

Figure 21: (A) Scatter plot showing the correlation between the $\mathrm{CH}_{4}$ integrated band depth in Ranges 1 (top), 2 (middle), and 4 (bottom) with the NSS endmember areal mass fraction. A moderate negative trend between the abundance of the NSS endmember with the integrated band depths of $\mathrm{CH}_{4}$ in Range 1, Range 2, and Range 4 is observed. (B) Scatter plot showing the correlation between the $\mathrm{CH}_{4}$ integrated band depth in Range 3 (top), at 1.48 $\mu \mathrm{m}$ (middle), and the $\mathrm{N}_{2}$ integrated band depth at $2.15 \mu \mathrm{m}$ (bottom) with the NSS endmember areal mass fraction. The NSS endmember is not correlated with the $\mathrm{CH}_{4}$ in Range 3, while it has a moderate and slight anti correlation with the $\mathrm{CH}_{4}$ band at $1.48 \mu \mathrm{m}$ and the $\mathrm{N}_{2}$ absorption at $2.15 \mu \mathrm{m}$. 


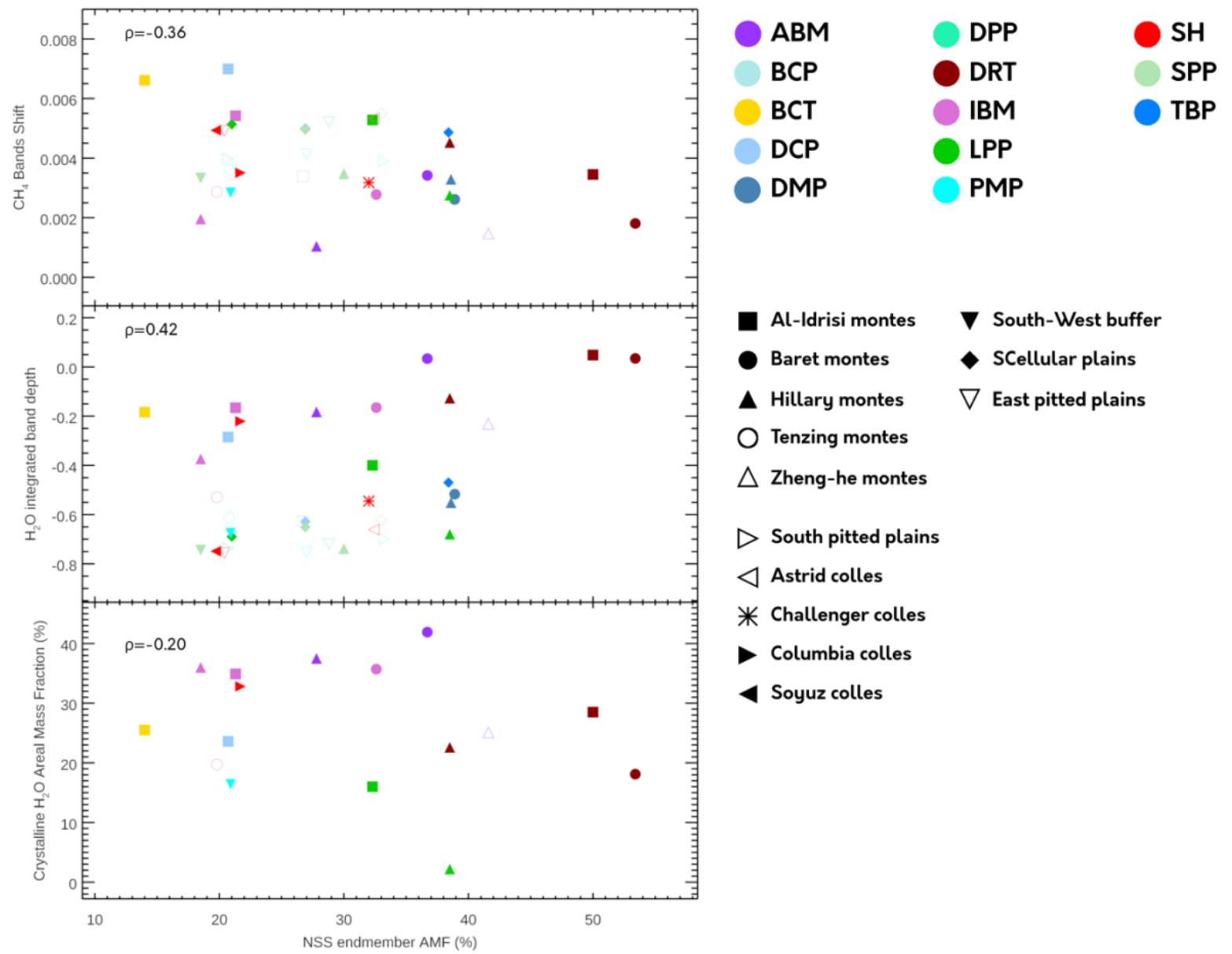

Figure 22: Scatter plot showing the correlation between the $\mathrm{CH}_{4}$ band shift index (top), $\mathrm{H}_{2} \mathrm{O}$ integrated band depth (middle), and crystalline $\mathrm{H}_{2} \mathrm{O}$ areal mass fraction (bottom) versus the NSS endmember areal mass fraction. The top panel shows a slight dependence between the shift value and the NSS slope, while in the other two panels, the represented quantities do not show any clear correlation. 


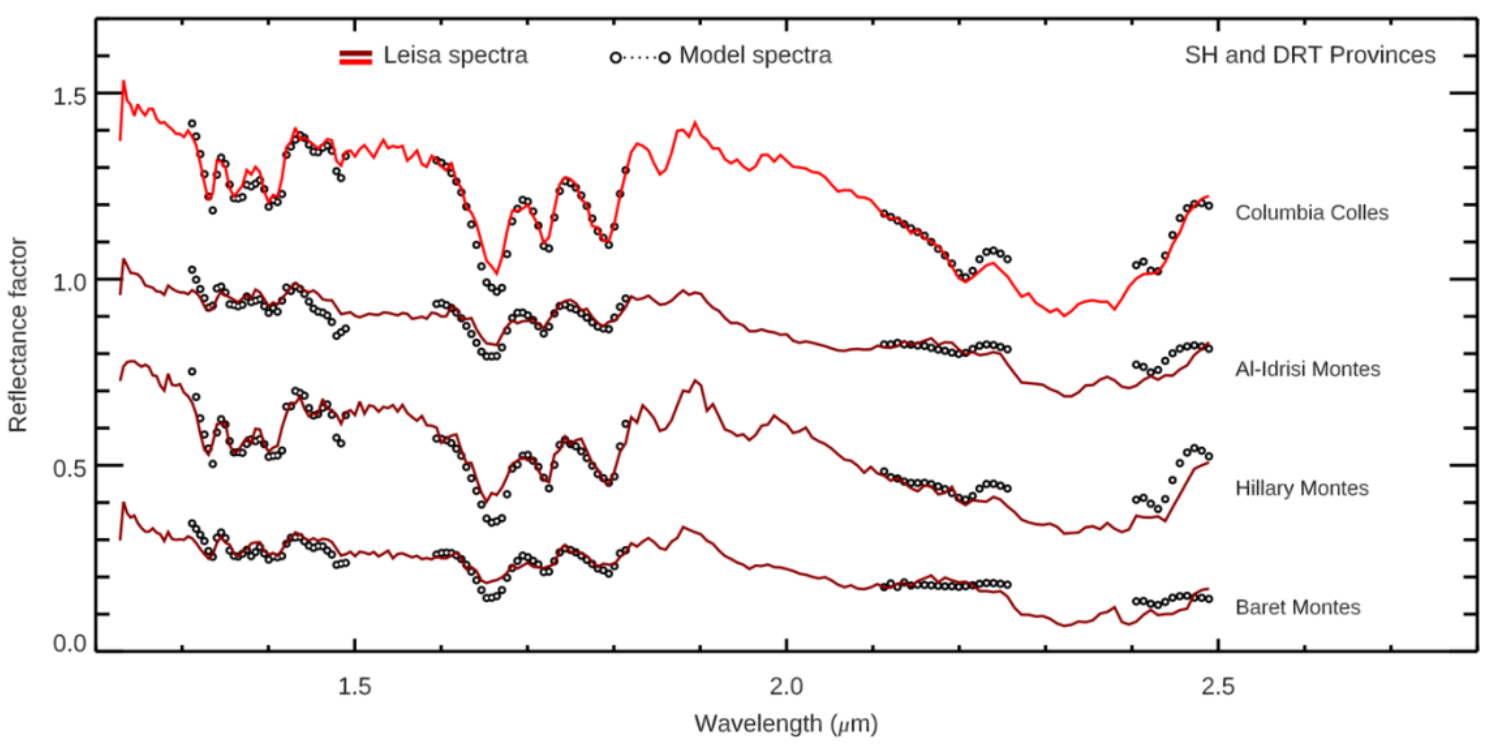

Figure 23: LEISA spectra, averaged for unit drt in Al-Idrisi, Hillary and Baret montes, and unit sh in Columbia Colles, overlapped by the corresponding Hapke theory-based best fit model. In this case, the modeling made use of the Pluto tholins optical constants, in place of the NSS endmember ones. The spectra are offset for clarity.

\section{Geological units composition of provinces}

\section{Baret Montes (b)}
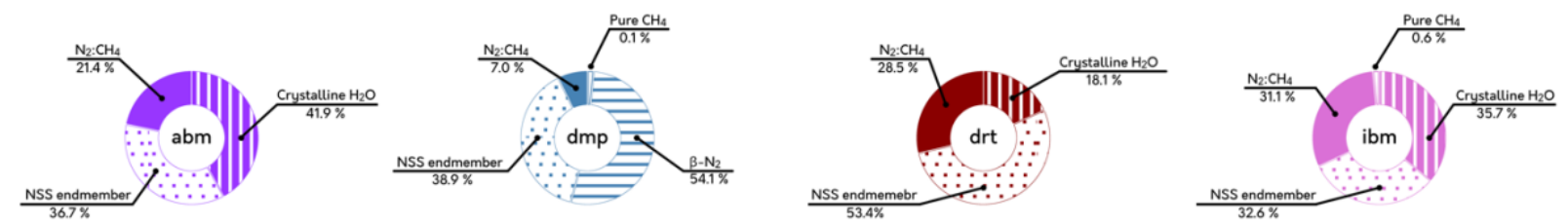


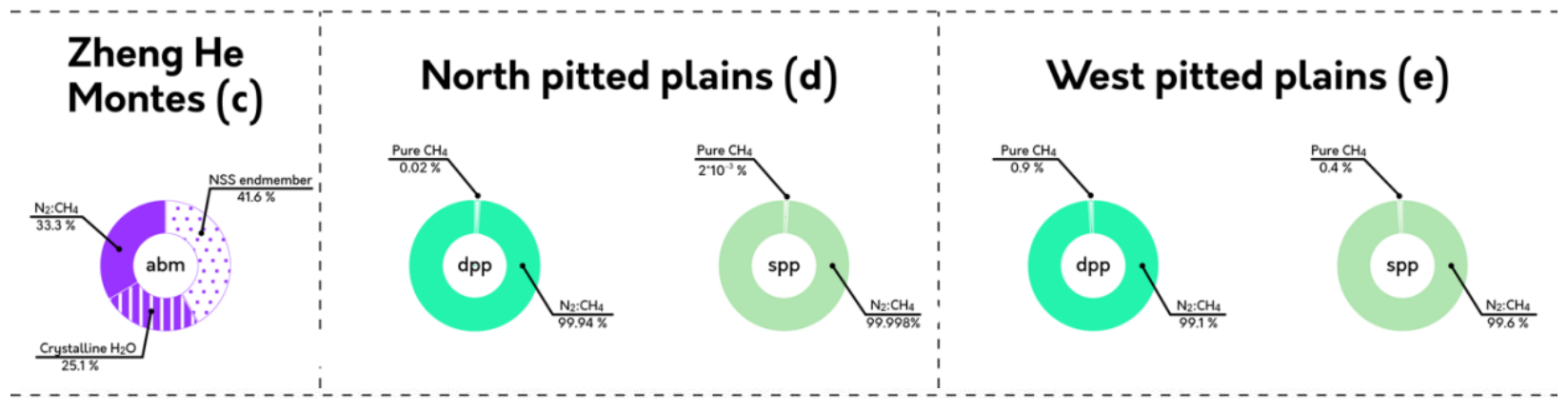

Hillary Montes (f)

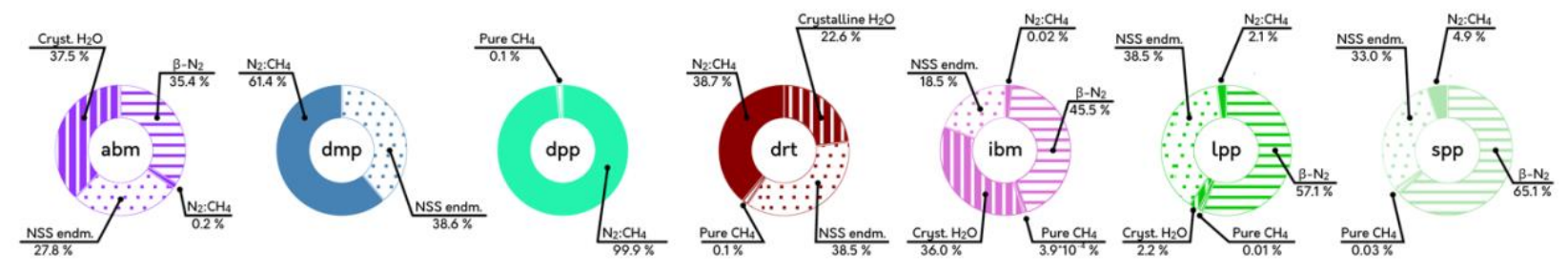

\section{Cellular plains (g)}
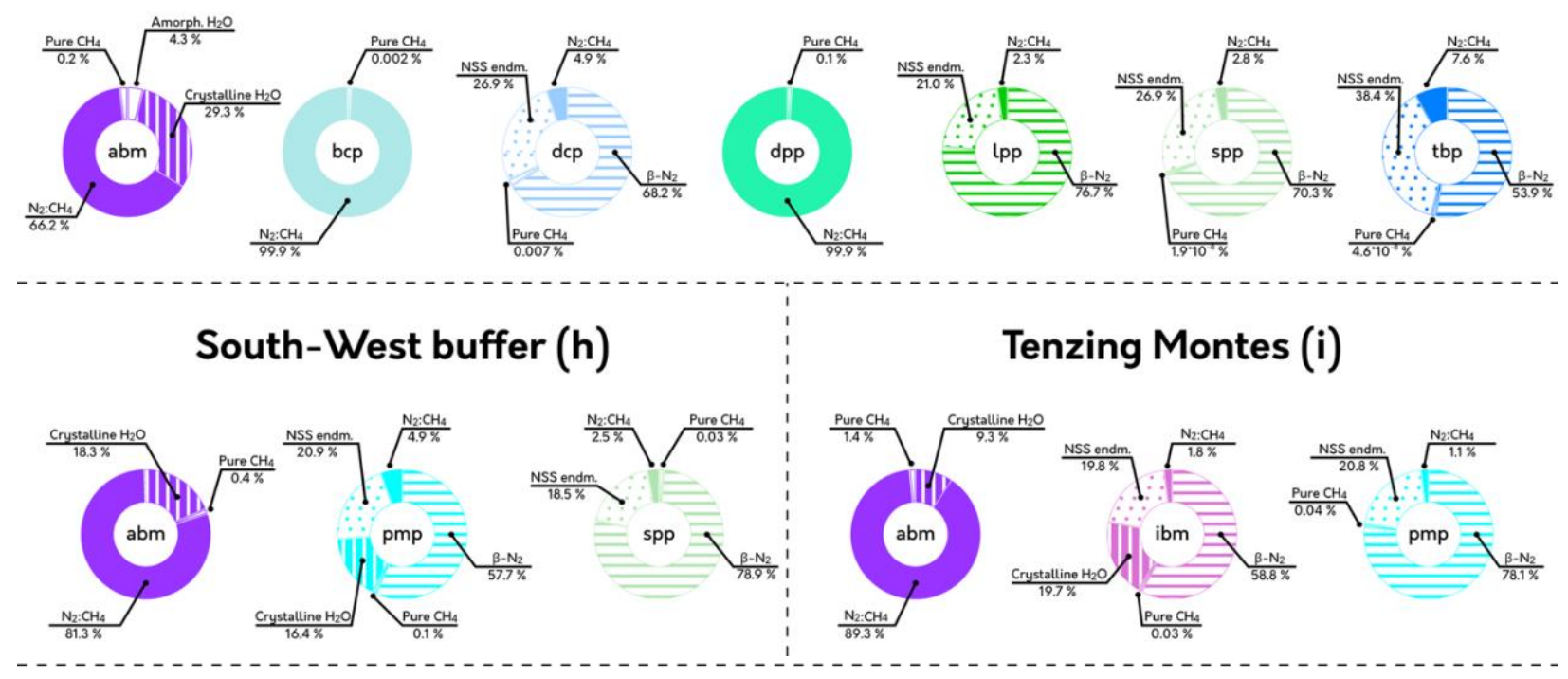

\section{East pitted plains (j)}
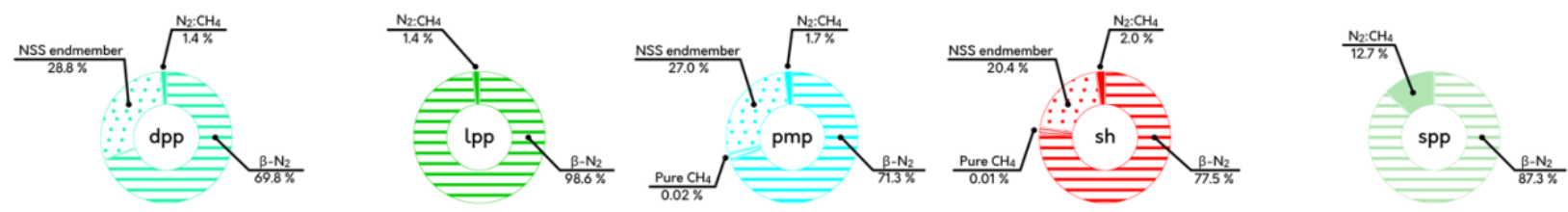


\section{South pitted plains (k)}
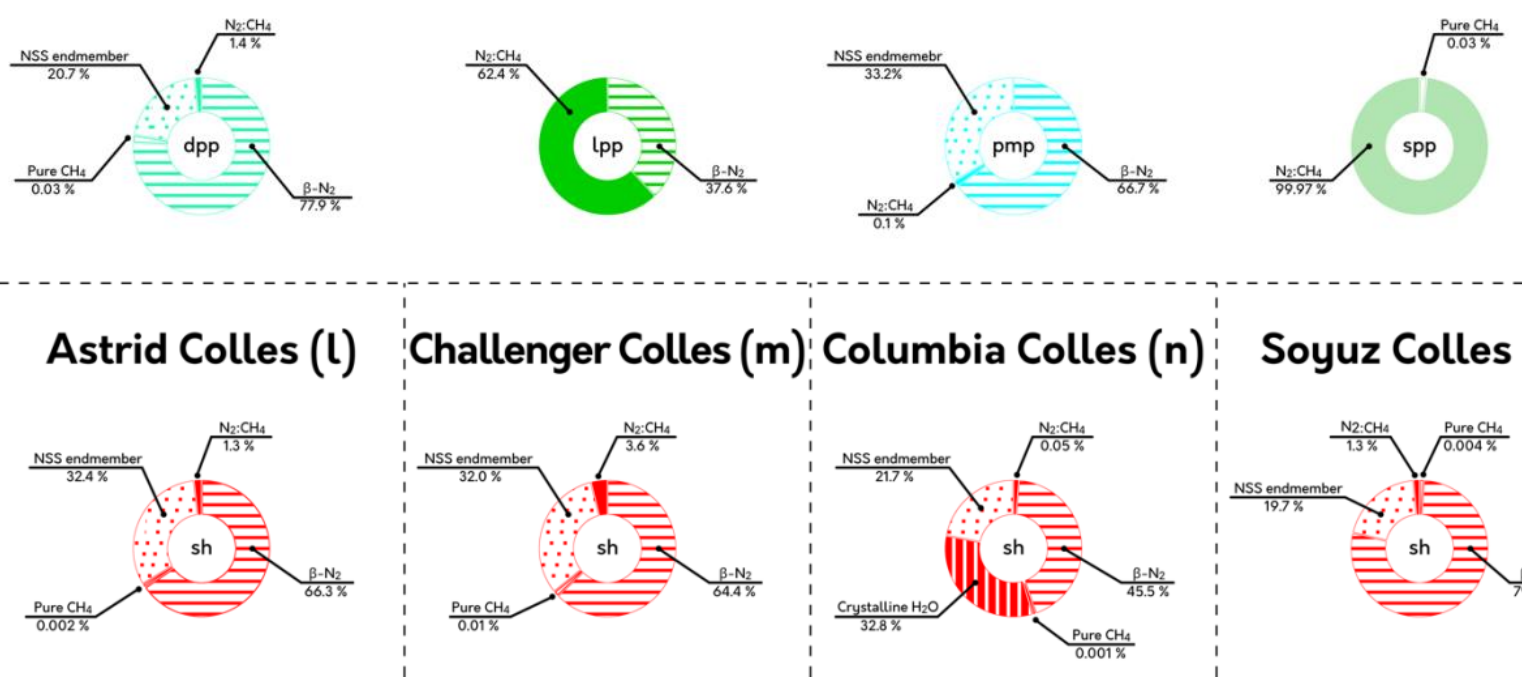

Soyuz Colles (0)

Figure 24 A-O: Pie plots summarizing the composition of the geologic units in each province, as returned by the application of the Hapke-based spectral modeling.

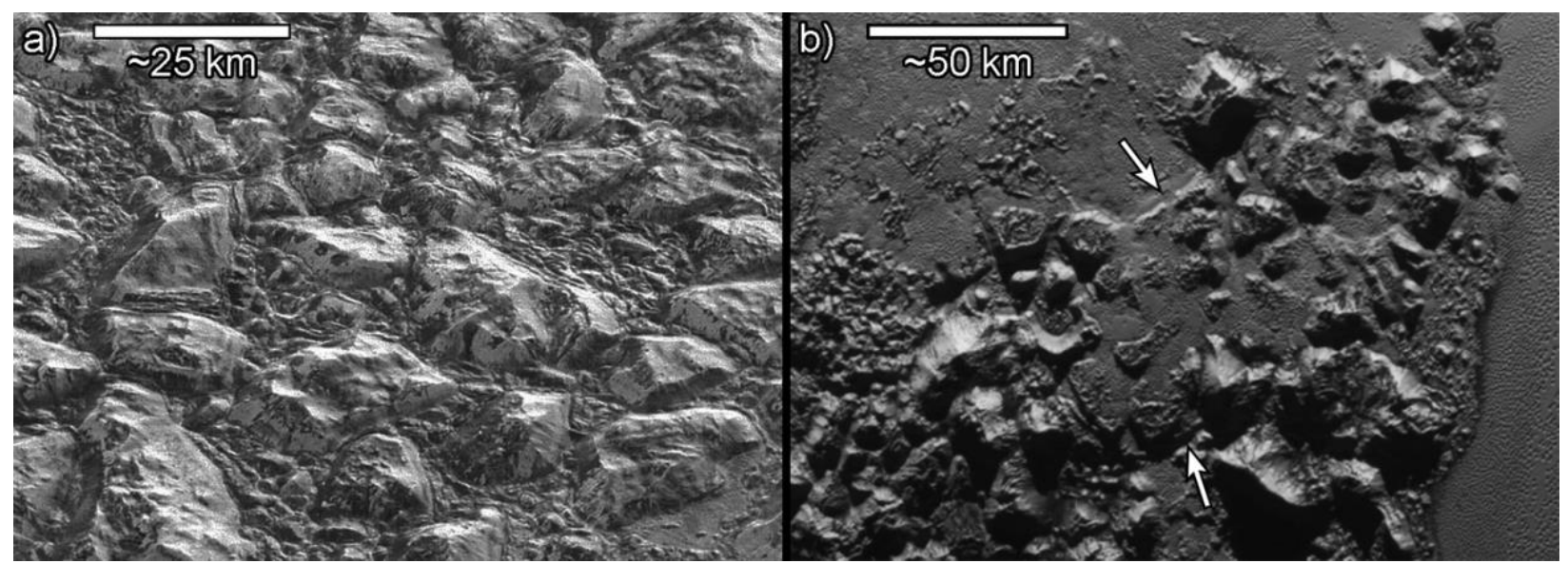

Figure 25: Contrast between the northernmost mountain range of Al-Idrisi Montes and the southernmost range of Tenzing Montes. (a) Al-Idrisi Montes show minimal interstitial N2 ice plains between mountain blocks. LORRI image is $76 \mathrm{~m} /$ pixel and centered at $37.6^{\circ} \mathrm{N}, 156.3^{\circ} \mathrm{E}$, with illumination from the top. (b) Several of the mountain blocks of Tenzing Montes are partly engulfed by $N 2$ ice plains that are elevated by up to a kilometer above the surrounding N2 ice plains of Sputnik Planitia, and separated from them by scarps (white arrows). MVIC image is $315 \mathrm{~m} /$ pixel and centered at $13.6^{\circ} \mathrm{S}, 176.7^{\circ} \mathrm{E}$, with illumination from the top. 


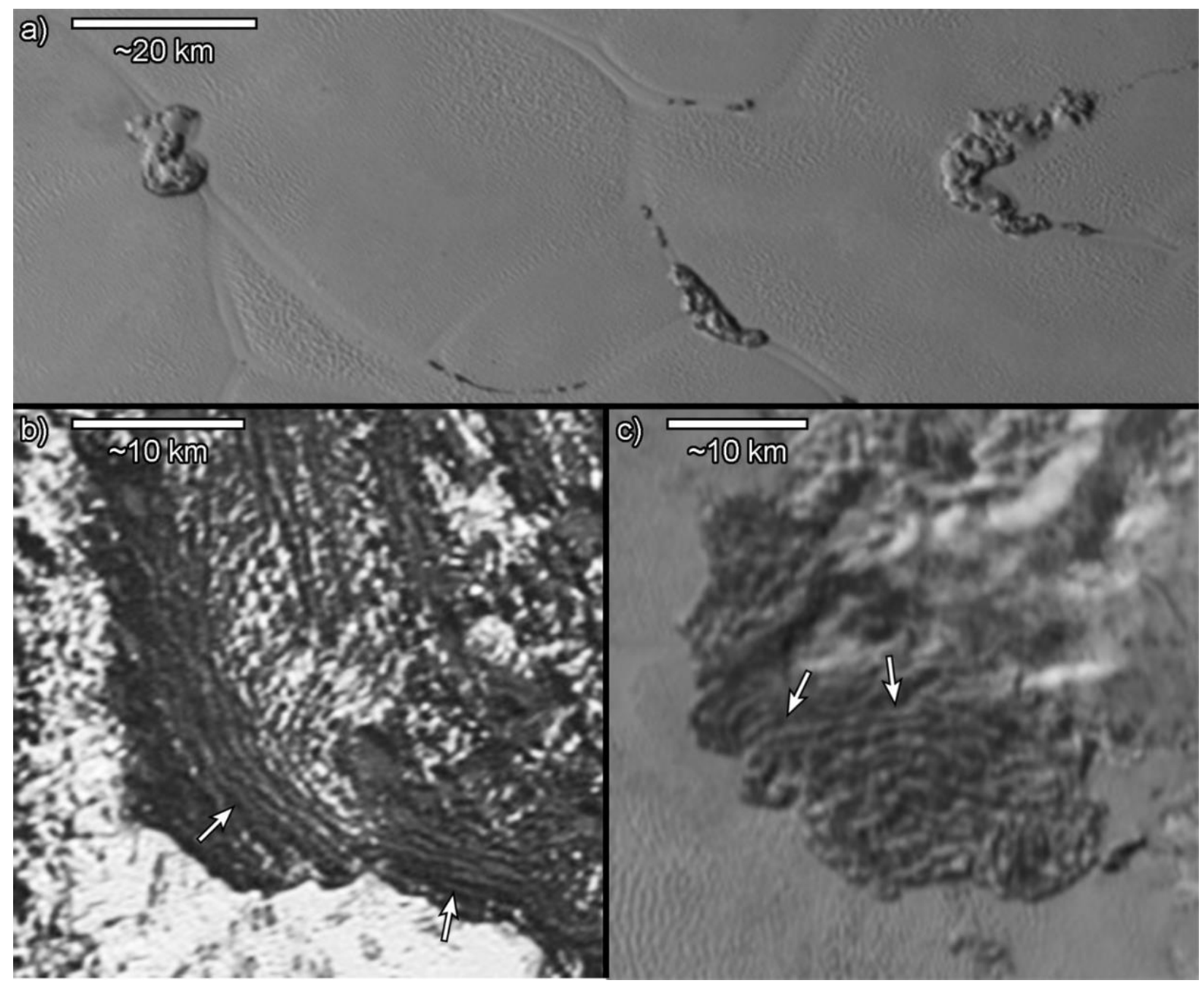

Figure 26: Scattered hills (unit sh) and dark ridged terrain (unit drt). (a) Scattered hills of Astrid and Soyuz Colles, which display smooth topographic relief and a high albedo, indicating that the hills, like the surrounding plains, have been coated by ongoing $\mathrm{N}_{2}$ ice deposition. MVIC image is $315 \mathrm{~m} /$ pixel and centered at $14.7^{\circ} \mathrm{N}, 184.5^{\circ} \mathrm{E}$, with illumination from the left. (b) Dark ridges situated between Baret Montes and dark-pitted, marginal plains of Sputnik Planitia, which were mapped as unit drt by White et al. (2017). White arrows indicate ridges spaced 1-2 km apart that align roughly parallel to the border of the unit with the $N_{2}$ ice plains. LORRI image is $117 \mathrm{~m} /$ pixel and centered at $11.4^{\circ} \mathrm{N}, 157.5^{\circ} \mathrm{E}$, with illumination from the top. (c) Columbia Colles, located at the eastern margin of Sputnik Planitia, which were mapped as unit sh by White et al. (2017). White arrows indicate ridges spaced 1-2 km apart that resemble those highlighted in (a). The comparable geomorphology and albedo of Columbia Colles to occurrences of unit drt in the west suggest that similar processes have affected Columbia Colles, which are otherwise separated stratigraphically and compositionally from unit drt. MVIC image is $315 \mathrm{~m} /$ pixel and centered at $28.7^{\circ} \mathrm{N}, 196.5^{\circ} \mathrm{E}$, with illumination from the left. 


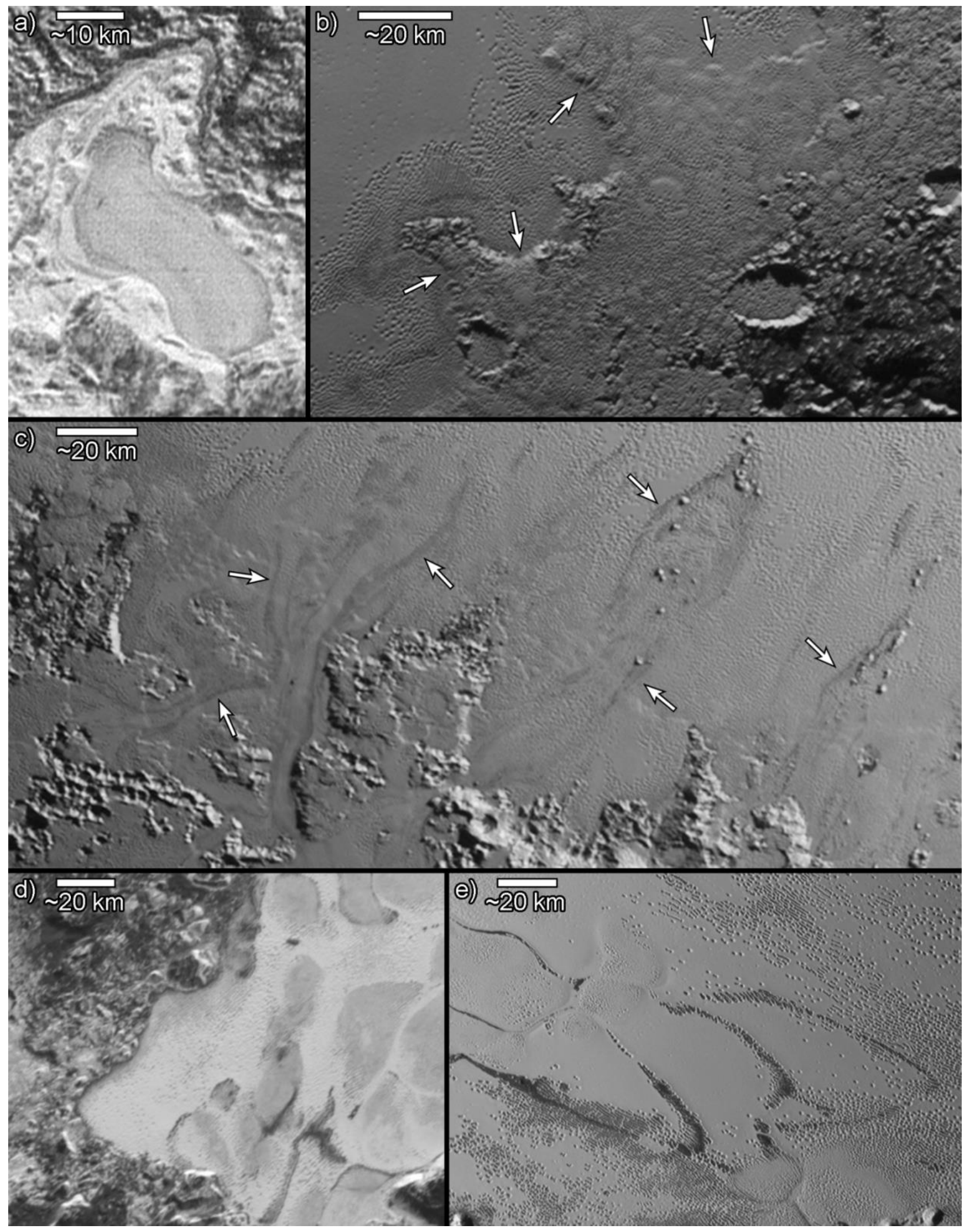

Figure 27: Plains units of Sputnik Planitia described in sections 5.5 and 5.6. (a) Alcyonia Lacus, mapped by White et al. (2017) as an isolated occurrence of dark cellular plains surrounded by mountains and blocky material of Al-Idrisi Montes. LORRI image is $117 \mathrm{~m} /$ pixel and centered at $36.4^{\circ} \mathrm{N}, 151.6^{\circ} \mathrm{E}$, with illumination from the top-right. (b) 
Hummocky $\mathrm{N}_{2}$ ice plains of unit pmp covering low-lying uplands of Krun Macula (dark terrain at lower right). Scarps (white arrows) separate unit pmp from the flat plains of Sputnik Planitia, similar to those seen at Tenzing Montes (Fig. 26b). MVIC image is $315 \mathrm{~m} /$ pixel and centered at $5.8^{\circ} \mathrm{S}, 191.2^{\circ} \mathrm{E}$, with illumination from the left. (c) Glacial flow of nitrogen ice from the bright pitted uplands of East Tombaugh Regio (bottom) into Sputnik Planitia. Dark, converging flow bands (white arrows) are seen in valleys that connect upland flats to Sputnik Planitia, and are interpreted to be moraine trails consisting of $\mathrm{CH}_{4}$-rich material eroded from the pitted uplands by $\mathrm{N}_{2}$ ice glaciation. MVIC image is $315 \mathrm{~m} /$ pixel and centered at $3.7^{\circ} \mathrm{N}, 193.6^{\circ} \mathrm{E}$, with illumination from the top-right. (d) High-albedo, pitted plains of units $d p p$ and spp located at the western edge of Sputnik Planitia, occurring interstitially to convective cells of the dark, cellular plains (unit dcp). MVIC image is $315 \mathrm{~m} /$ pixel and centered at $20.3^{\circ} \mathrm{N}, 155.1^{\circ} \mathrm{E}$, with illumination from the top. (e) Dense fields of aligned (unit dpp) in southern Sputnik Planitia. Across the center of the image, they are seen to form chains of joined, dark-floored pits that trace elongated, polygonal outlines that enclose sparsely pitted plains (unit spp), and which extend southwards from the bright, cellular plains (unit bcp) at top left. MVIC image is $315 \mathrm{~m} /$ pixel and centered at $6.0^{\circ} \mathrm{S}, 179.7^{\circ} \mathrm{E}$, with illumination from the left.
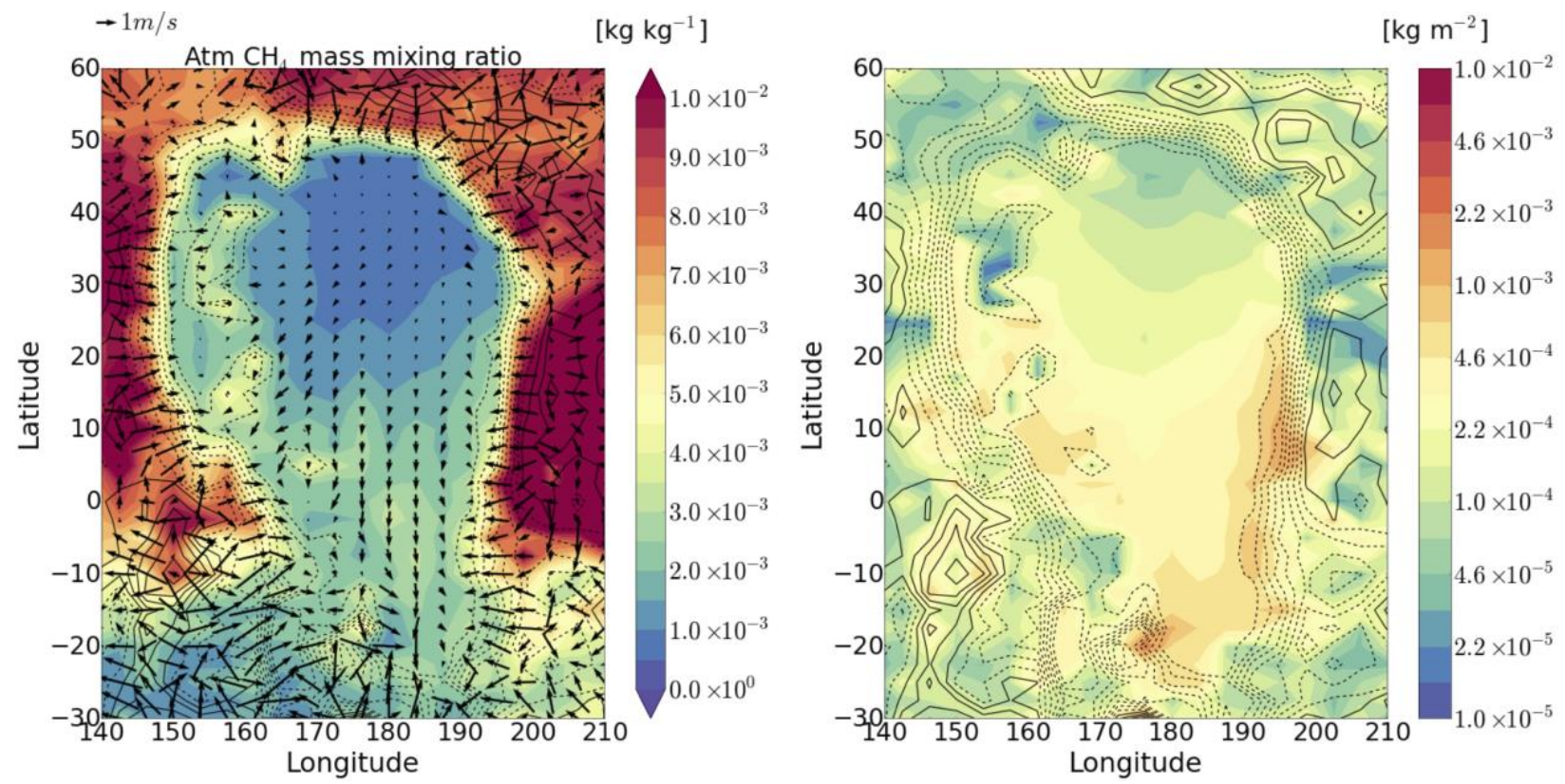

Figure 28: Results from the global climate model of Pluto obtained for July 2015 from simulations similar to those presented in Bertrand et al., 2020b. Left: Diurnal mean near-surface ( $20 \mathrm{~m}$ ) atmospheric $\mathrm{CH}_{4}$ mass mixing ratio in the region of Sputnik Planitia, with near-surface winds. Right: Net diurnal $\mathrm{CH}_{4}$ deposition onto the surface. 